
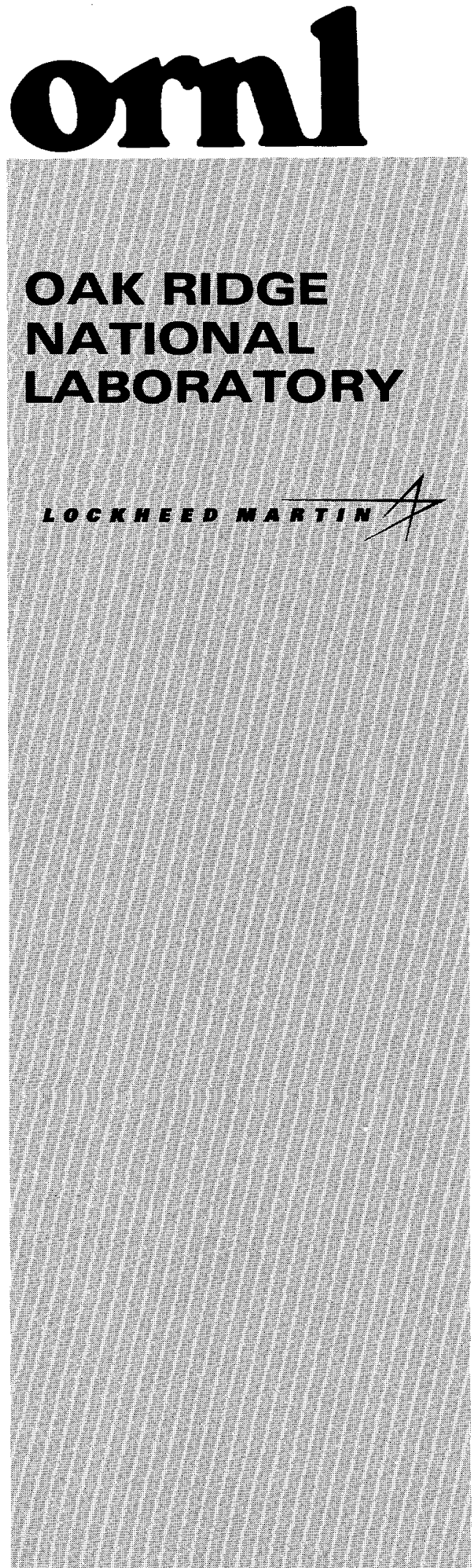

MANAGED AND OPERATED BY LOCKHEEO WARTIN ENERGY RESEARCH COBPORATION FOR THE UNTED STATES DEPARTIENT OF ENERGY

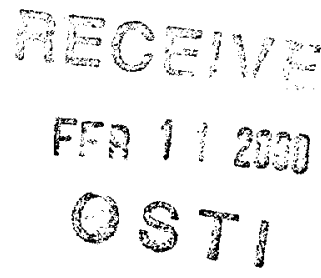

Next Generation Grinding Spindle for

Cost-Effective Manufacture of

Advanced Ceramic Components

FINAL REPORT

Joseph A. Kovach

Michael A. Laurich 
This report has been reproduced from the best available copy.

Reports are available to the public from the following source.

National Technical Information Service

5285 Port Royal Road

Springfield, VA 22161

Telephone 703-605-6000 (1-800-553-6847)

TDD 703-487-4639

Fax 703-605-6900

E-mail orders@ ntis.fedworid.gov

Web site http://www.ntis.gov/ordering.htm

Reports are available to U.S. Department of Energy (DOE) employees, DOE contractors, Energy Technology Data Exchange (ETDE) representatives, and International Nuclear Information System (INIS) representatives from the following source.

Office of Scientific and Technical Information

P.O. Box 62

Oak Ridge, TN 37831

Telephone 423-576-8401

Fax 423-576-5728

E-mail reports@ adonis.osti.gov

Web site http://www.osti.gov/products/sources.html

Reports produced after January 1,1996 , are generally available via the DOE Information Bridge.

Web site http://www.doe.gov/bridge 


\section{DISCLAIMER}

This report was prepared as an account of work sponsored by an agency of the United States Government. Neither the United States Government nor any agency thereof, nor any of their employees, make any warranty, express or implied, or assumes any legal liability or responsibility for the accuracy, completeness, or usefulness of any information, apparatus, product, or process disclosed, or represents that its use would not infringe privately owned rights. Reference herein to any specific commercial product, process, or service by trade name, trademark, manufacturer, or otherwise does not necessarily constitute or imply its endorsement, recommendation, or favoring by the United States Government or any agency thereof. The views and opinions of authors expressed herein do not necessarily state or reflect those of the United States Government or any agency thereof. 


\section{DISCLAIMER}

Portions of this document may be illegible in electronic image products. Images are produced from the best available original document. 


\title{
NEXT GENERATION GRINDING SPINDLE FOR COST-EFFECTIVE MANUFACTURE OF ADVANCED CERAMIC COMPONENTS
}

Dr. Joseph A. Kovach, P.E, Director, Manufacturing Process Technology and

Michael A. Laurich

Date Published:

\section{FINAL REPORT}

\author{
Prepared by \\ Eaton Corporation \\ Manufacturing Technologies Center \\ Willoughby Hills, Ohio
}

Funded by

U.S. Department of Energy

Assistant Secretary for Energy Efficiency and Renewable Energy

Office of Transportation Technologies

Heavy Vehicle Propulsion System Materials Program

EE 0701000

for

OAK RIDGE NATIONAL LABORATORY

Oak Ridge, Tennessee 37831-6285

Managed by

LOCKHEED MARTIN ENERGY RESEARCH CORPORATION

for the

U.S. DEPARTMENT OF ENERGY

under Contract DE-AC05-96OR22464 


\section{TABLE Of CONTENTS}

ABSTRACT

1.0 INTRODUCTION and OBJECTIVES . . . . . . . . . . 2

2.0 BACKGROUND . . . . . . . . . . . . . . . 4

3.0 TECHNICAL APPROACH . . . . . . . . . . . 8

4.0 RESULTS and DISCUSSION . . . . . . . . . . . . . . . 10

4.1 Overall Spindle Configuration. . . . . . . . . . . . 10

4.2 Bearing Design \& Predicted Performance . . . . . . . . 11

4.2.1 Radial Bearing . . . . . . . . . . . . . . . 12

4.2.2 Thrust Bearing . . . . . . . . . . . . . 15

4.3 Motor/Drive Design \& Predicted Performance. . . . . . . . 16

4.3.1 Motor Design \& Selection . . . . . . . . . . . . . 17

4.3.2 Drive Design \& Selection . . . . . . . . . . . 18

4.3.2.1 Drive Operation . . . . . . . . . . . 19

4.3.2.2 Principle of Sensorless Commutation. . . . . . . 20

4.3.3 Spindle/Motor Thermal Analysis. . . . . . . . . . 20

4.4 Ancillary Component Design Considerations . . . . . . . . 21

4.4.1 Shaft . . . . . . . . . . . . . . 22

4.4.2 Hub and Wheel . . . . . . . . . . . . . . . 22

4.4.3 Side Plates . . . . . . . . . . . . . 23

4.4 .4 Seals. . . . . . . . . . . . . 23

4.4 .5 Balancing . . . . . . . . . . . . . . . 24 
5.0 SUMMARY. . . . . . . . . . . . . . . . . 25

6.0 ACKNOWLEDGMENTS . . . . . . . . . . . . . . 27 
iii

\section{LIST of TABLES}

TABLE

PAGE

1 Spindle comparison chart 28

$\begin{array}{lll}2 & \text { Bearing predicted performance } & 29\end{array}$

3 Effects of centrifugal loads on side plate/journal 29 


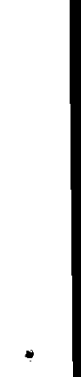




\section{LIST of FIGURES}

\section{FIGURE}

PAGE

$1 \quad$ Viking centerless grinder - "Twin-grip" spindle arrangement

2 Conceptual spindle arrangement in Modler centerless grinder

3 Spindle concept

$4 \quad$ Next generation spindle - overall concept model

5 Stationary shaft detail

6 DC motor stator detail

$7 \quad$ Tilting pad fluid bearing detail 36

8 Motor rotor wheel assembly 37

$9 \quad$ Bearing journal radial expansion versus operating speed 38

$\begin{array}{ll}10 \text { Flanged bearing concept } & 39\end{array}$

11 Deformation of flanged bearing under 7,000 RPM operation 40

12 Deformation of flanged bearing under hydrodynamic fluid pressure

13 Bearing design and operating characteristics

14 Effect of shaft misalignment on bearing load capacity

15 Required preload to maintain constant stiffness over speed range 44

16 Minimum film thickness versus operating speed 45

17 Power lost in bearing versus operating speed 46

18 Oil flow rate as a function of operating speed 47

19 Peak film temperature as function of operating speed 48

20 Connection schematic $\quad 49$ 


\section{LIST of FIGURES (cont'd.)}

FIGURE

PAGE

21 Winding specifications

50

22 Predicted temperature distribution of spindle under full power

51

23 Separation of two-piece side plate under 7,000 RPM operation

52 


\section{DRAWING}

\section{LIST of DRAWINGS}

1 Overall assembly

PAGE

53

2 Bearing design (Overall layout) 54

3 Bearing design details - thrust and radial pads 55

$4 \quad$ Tilting pad assembly (tilt on fixed location pivot) 56

5 Tilting pad assembly (tilt on actuator) 57

$6 \quad$ Tilting pad detail $\quad 58$

$\begin{array}{lll}7 & \text { Fixed location pad detail } & 59\end{array}$

8 Actuator/Pivot detail 60

$9 \quad$ Lamination stack assembly with typical lamination 61

10 Motor assembly schematic (stator and rotor end view) 62

11 Motor hub with skewed magnet 63

12 Block diagram of spindle motor drive 64

13 Motor cooling sleeve - cooling passages 65

14. Motor cooling sleeve - clamping flange 66

15 Spindle assembly end view (motor, shaft, cooling sleeve, wheel) 67

16 Shaft detail - conduits for bearing, oil, cooling and motor leads 68

17 Cooling sleeve sealing ring 69

$\begin{array}{ll}\text { 18a Side plate - right side } & 70\end{array}$

18b Side plate - left side $\quad 71$

18c Side plate seal clamp $\quad 72$

18d Wheel clamp 73

19 Assembly view of end plate, bearing, actuator and seals 74 


\author{
NEXT GENERATION GRINDING SPINDLE \\ FOR \\ COST-EFFECTIVE MANUFACTURE \\ OF \\ ADVANCED CERAMIC COMPONENTS
}

\begin{abstract}
Finish grinding of advanced structural ceramics has generally been considered an extremely slow and costly process. Recently, however, results from the High-Speed, Low-Damage (HSLD) program have clearly demonstrated that numerous finish-process performance benefits can be realized by grinding silicon nitride at high wheel speeds. A new, singlestep, roughing-finishing process capable of producing high-quality silicon nitride parts at high material removal rates while dramatically reducing finishing costs has been developed. To take full advantage of the benefits of HSLD grinding for large-volume ceramic production, this technology must be extended to centerless grinding operations. The subject effort focused on the design of a Next Generation Spindle (NGS) for HSLD centerless applications, specifically a $50 \mathrm{hp}$, twin-grip, centerless grinding spindle capable of using 12-14-in.-diameter by 8-12in.-wide grinding wheels with at least $7,00 \mathrm{~h}$-rpm capability in a throughfeed or in-feed grinding mode. The design spindle stiffness was to be $2,000,000 \mathrm{lb} / \mathrm{in}$. minimum. Accordingly, the work described here involved the design of a novel fluid film spindle bearing arrangement as well as an integral spindle/motor package.
\end{abstract}

Research sponsored by the U.S. Department of Energy, Assistant Secretary for Energy Efficiency and Renewable Energy, Office of Transportation Technologies, as part of the Heavy Vehicle Propulsion System Materials Program, under contract DE-AC05-96OR22464 with Lockheed Martin Energy Research Corporation. 


\subsection{INTRODUCTION and OBJECTIVES}

Finish grinding of advanced structural ceramic components has generally been considered to be an extremely slow and costly process. Typically, the mechanical and physical characteristics which make these materials desirable from a product performance standpoint usually render them far from ideal in terms of manufacturability. Recently, however, the Oak Ridge National Laboratory program on High-Speed, Low-Damage (HSLD) grinding clearly demonstrated that numerous finish process performance benefits can be realized by grinding silicon nitride at high wheel speeds. More specifically, it was shown that increasing wheel speeds will:

- Reduce grinding forces

- Improve surface finish

- Increase wheel life
- Reduce bulk workpiece temperatures

- Allow for high material removal rates

- Meet or exceed all baseline material properties

- Significantly reduce total finishing costs

Essentially, the results of the Oak Ridge HSLD program lead to the development of a new single step, roughing-finishing process capable of producing high-quality silicon nitride ceramic parts at high material removal rates while dramatically reducing finishing costs. However, to take full advantage of the benefits of High-Speed, Low-Damage (HSLD) grinding for large volume ceramic production, the HSLD technology must be extended to centerless grinding operations. Unfortunately, when considering all the centerless grinding machine elements, the lack of a high speed, high stiffness twin grip spindle reduces HSLD implementation potential.

In this regard, this program is focused on the design of a Next Generation Spindle (NGS) ideally suited for HSLD centerless applications. It is anticipated that major advances in cost effective ceramic finishing will result from centerless grinding at high material removal rates while utilizing the low damage (HSLD) grinding process. The overall design philosophy behind this effort is to use small diameter diamond wheels operating at high speeds. Such an approach will reduce wheel inventory costs and machine floor space requirements while minimizing the downside consequences of an in-process wheel crash. More specifically, the objective of this program is to design a "twin grip" (i.e. grinding wheel is rigidly supported at each end), high speed, centerless grinding spindle capable of operating under High-Speed, Low-Damage grinding conditions. The particular tasks to be addressed include:

- Design of a twin grip spindle bearing arrangement capable of 7,000 $\mathrm{rpm}, 50 \mathrm{HP}$, and a minimum stiffness of $2,000,000 \mathrm{lbs} / \mathrm{in}$.

- Design of a spindle to accept a 12" to 14 " diameter, 8" to 12 " wide grinding wheel that will operate in an in-feed as well as a through-feed mode. 
- Design an integral spindle motor with a constant torque from 0 to $2,000 \mathrm{rpm}$, and a constant 50 horsepower from 2,000 to 7,000 rpm.

- Design a wheel hub and spindle/motor package that will utilize as small an area as possible while allowing for rapid change-over and accurate rotational characteristics (e.g., low run-out and vibration).

In addition, sensors for monitoring spindle speed, vibration, and temperature are to be selected and incorporated into the design of the spindle. Methods for sealing parts of the machine from the harmful effects of fine grinding debris and coolant fluids will be incorporated into the final design. Any necessary support hardware, such as chillers, filters, air supply, and fluid separators will also be designed or selected and integrated into the final spindlesystem design. It is felt that aside from achieving the proven benefits of HSLD grinding (i.e. improved part quality and throughput), the eventual implementation of this Next Generation Spindle (NGS) will also result in increased production flexibility and cost savings by reducing the total number of centerless grinding machines/operations required. 


\subsection{BACKGROUND}

Over the past several years it has become increasingly apparent that the use of elevated grinding wheel speeds (i.e. over 20,000 SFM) can lead to dramatic increases in material removal rates and grinding wheel life. Initially, the High Speed Grinding (HSG) phenomenon was industrialized in limited applications utilizing conventional aluminum oxide wheels for grinding ferrous alloys. However, due to the strength limitations of conventional vitreous bonded grinding wheels, peripheral speeds seldom exceed 24,000 SFM. Subsequently, with the increased commercial acceptance of Cubic Boron Nitride (CBN) superabrasives, some production applications are now using plated $\mathrm{CBN}$ wheels at speeds approaching 80,000 SFM (1.3 times the speed of sound). Most recently, through efforts initiated by Oak Ridge National Laboratory, it has been demonstrated that HSG principles can be applied to the finishing of silicon nitride with considerable improvements in material removal rate, wheel life, and resulting surface quality.

One of the key results of this ORNL program on High-Speed, LowDamage (HSLD) grinding showed that increasing wheel speed will reduce diamond wheel wear. At $1.0 \mathrm{in}^{3} / \mathrm{min} /$ in material removal rate, increasing wheel speed from 6,000 to $18,000 \mathrm{ft} / \mathrm{min}$ produced over a three fold increase in wheel life. Even at this high removal rate the resulting wheel wear using high wheel speeds was only about half of that observed using conventional removal rates $\left(0.1 \mathrm{in}^{3} / \mathrm{min} / \mathrm{in}\right)$ and wheel speeds $(6,000 \mathrm{sfm})$. Alternatively stated, increasing the wheel speed from 6,000 to $18,000 \mathrm{ft} / \mathrm{min}$ allowed for a 10 fold increase in material removal rate while cutting wheel wear in half.

In addition, the HSLD program also demonstrated that increasing wheel speeds will generally reduce grinding forces, improve surface finish, reduce "pull out" or surface fragmentation, and reduce bulk workpiece temperatures. Numerous tests were also performed to identify the relationship between increasing wheel speed and resulting MOR strength. For all wheels tested it was shown that as wheel speed is increased, the flexural strength is also increased. For example, at 18,000 SFM a 240 grit resin bonded wheel produced much higher quality parts than those generated under slow MIL-STD-1942 conditions - - while grinding at material removal rates that were over 10 times greater than that used in baseline MIL-STD-1942 conditions. Even grinding with a coarse 180 grit resin wheel at 18,000 sfm produced parts which exceeded baseline properties while grinding at over 10 times the MIL-STD-1942 removal rates.

To characterize overall HSLD cost performance, a comprehensive cost model was also developed to predict grinding related costs for a single plunge centerless grinding operation over a range of material removal rates and wheel speeds. In all cases increasing wheel speed reduced costs. Moreover, the cost benefits gained by increased wheels speeds outweighed those achieved from reducing labor rates. It was shown that the final per piece costs obtained at $18,000 \mathrm{sfm}$ using a high labor rate are actually lower than those using a conventional wheel speed of $6,000 \mathrm{sfm}$ and a low labor rate. Under simulated 
production conditions, increasing the wheel speed from 6,000 to 18,000 SFM on a standard 240 grit resin wheel resulted in a 3 -fold increase in wheel life while exceeding baseline material properties and reducing total per piece finishing costs by a factor of three.

Based on this cost model it became quite clear that the most dominant costs arise from the abrasive cost contribution On the other hand, the cost contribution from capital is minimal. With this in mind, the obvious "least cost" production strategy is to maximize wheel speeds through utilization of state-ofthe-art capital. Recognize, however, that the majority of potential ceramic heat engine components are cylindrical and would be typically finished in large volumes using centerless grinding equipment. Therefore, to fully exploit this technology for high volume cylindrical component finishing, centerless grinding machines will require new high-speed, high-stiffness spindles. Unfortunately, until ceramic production reaches high volumes, it is unlikely that machine tool vendors will design/build a centerless grinder for high throughput grinding of advanced ceramics.

It is important to note that as a whole, centerless grinders still operate as they were originally designed; with slow, fluid film spindles, turning large diameter (20"-24") aluminum oxide grinding wheels at speeds on the order of 5,000 SFM. Consequently, current centerless spindle technology actually impedes introduction of superabrasive (i.e. diamond and CBN) grinding processes to the production environment in the following areas:

- Wheel Speed - Superabrasive wheels operate best at high surface speeds. The higher the speed, the lower the forces and hence, higher material removal rates with less wheel wear. To achieve high wheel speeds on conventional spindles, large diameter wheels must be used, at prohibitively high tooling costs.

- Power - At high speed operation, material removal rates can be increased significantly. Current spindles lack sufficient power to take advantage of higher wheel speeds.

- Motor - Typically, the motor is connected to the spindle using pulleys and belts. These mechanical components add vibration to the system which is manifested as workpiece surface defects. The NGS spindle will be direct drive, greatly reducing system vibrations.

- Stiffness and Damping - Current spindles generally use angular contact ball bearings which have limited stiffness (at the cost of bearing life) and minimal damping capacity. Alternatively, old plain shell hydrodynamic bearings generally lack accuracy due to low stiffness. Hydrostatic bearings have shown promise at conventional speeds, but are costly due to the 1000+ PSI hydraulic support requirements. The NGS spindle will use high-speed, tilting-pad fluid film bearings, known for excellent damping capacity and high stiffness. 
- Space constraints - The sizes of the typical motor, pulleys, and belts increase the overall machine envelope. The NGS design goals promote an envelope just slightly larger than that occupied by the grinding wheel. As such, the grinding machines can be made smaller, reducing total floor space requirements.

In the machine tool vendor's defense, however, little has changed over the past 50 years to necessitate redesigning centerless grinders. Therefore, diamond wheel use will be severely limited. Consider, for example, that typical large diameter diamond grinding wheels can cost between $\$ 20,000$ to $\$ 100,000$. As such, wheel inventory costs and the economic consequences of a wheel "crash" can be extremely distressing. Also, as indicated above, common centerless machines tend to lack the power and rigidity necessary for cost effective grinding with diamond wheels. Hence, manufacturing "efficiency" is currently obtained by ganging three to five centerless grinders in a row which typically remove $0.001-.005$ " or less of material per machine. Obviously, this type of approach is unacceptable for use in an agile manufacturing environment which demands flexibility (i.e. no/low setup) as well as high throughput rates.

In an effort to develop a Next Generation Centerless (NGC) grinder for ceramic heat engine component production, the Eaton Corporation has conducted an in-depth investigation of the technologies currently limiting the development of such a machine. Of all the machine elements (e.g. base, slides, controls, etc.) it has become quite evident that the "heart" of any high performance centerless grinder is clearly the spindle. Without an adequate high speed, high stiffness spindle (which maximizes the performance capability of smaller diameter diamond grinding wheels in a user friendly fashion) it is unlikely that large volume HSLD ceramic finishing will reach its full potential. Based on preliminary tests, an HSLD diamond centerless grinding machine should have a spindle capable of operating at aggressive depths of cuts (0.010" to 0.020 ") using a 12 " to 14" diameter diamond wheel with peripheral speeds on the order of 25,000 SFM and a peak power of approximately $50 \mathrm{HP}$. Unfortunately, there are no commercial centerless grinder spindle manufacturers who presently sell, nor have plans to develop in the near term, such a spindle for high throughput grinding of advanced ceramics.

The need for a high speed, high stiffness, high accuracy "twin grip" centerless grinding spindle has been often discussed by spindle manufacturers. However, due to perceived market limitations, this type of spindle has not yet been developed. Numerous experts agree that if such a spindle were available, improved wheel life, decreased dress cycles, improved part finish and increased throughput would all be immediate benefits when using superabrasive grinding wheels.

In this study, a substantial portion of the background investigation included interviewing existing machine tool builders with in-house spindle design capabilities, second party spindle manufactures, as well as custom design companies. The Eaton investigation has concluded that several of the required technologies for this spindle have not yet been developed. Some of the OEM's 
have indicated that such a spindle technology development is necessary, but none have taken aggressive steps in that direction. The companies contacted included:

$\begin{array}{llll}\text { Cincinnati Milacron } & \text { Setco } & \text { Fisher } & \text { Whitnon } \\ \text { Gros-ite Spindles } & \text { Gilman } & \text { Pope } & \text { Ibag } \\ \text { Mikrosa Korber } & \text { Landis } & \text { Koyo } & \text { JBS } \\ \text { Ghiringhelli } & \text { Nissin } & \text { Gamfior } & \text { Goldcrown } \\ \text { Tschudin } & \text { Intermotion } & \text { Precise } & \text { John Brown } \\ \text { Barden } & \text { SKF } & \text { NSK } & \text { Smith-Renaud } \\ \text { Westwind Air Bearing Inc. } & \text { New England Affiliated Technologies } \\ \text { Professional Instruments Inc. } & \text { Boneham Precision Spindles } \\ \text { Seagull Precision Inc. } & & \text { Applied Grinding Technologies } \\ \text { Bryant Spindles } & & \text { Precision Balancing \& Analyzing }\end{array}$

The above list is both extensive as well as diversified. Nevertheless, the conclusion is clear that none of the above manufacturers currently have a spindle capable of satisfying the NGS specifications given earlier. Although most of these vendors did feel that an NGS spindle is necessary, their current work loads prohibited investing the type of resources necessary for supporting a 3-5 year commercialization effort. Smaller, slower spindles with less power are available. In this regard, most vendors would rather try to adapt existing off-theshell technology rather than starting with a "clean sheet of paper".

In addition to the vendor analysis, a published literature search was also conducted to determine what related research activity within academia could be applied. Although some dissociated research is at hand (high speed hydrostatic bearings, motors, etc.), a complete NGS package is not completely available. Consequently, the previous conclusion further supports the need to develop this NGS. If is felt that in addition to improving part quality and throughput, use of the Next Generation Spindle (NGS) will also result in increased flexibility and cost savings by reducing the number of machines required. 


\subsection{TECHNICAL APPROACH}

The primary program deliverable is the design and analysis of a Next Generation Spindle (NGS) for high speed centerless grinding of structural ceramic components. The individual components that comprise the NGS were designed through the combined use of mathematical models, finite element analysis, and solid modeling. The project team consisted of representatives from the following companies:

\begin{tabular}{|c|c|}
\hline $\begin{array}{l}\text { Overall Design } \\
\text { Fluid Film Bearings }\end{array}$ & $\begin{array}{l}\text { Eaton, Manufacturing Technologies Center (MTC) } \\
\text { Case Western Reserve University (Dr. M. Adams), } \\
\text { Eaton MTC, and KMC }\end{array}$ \\
\hline Motor Design & MotorSoft (J. Hendershot) \\
\hline Motor Magnets & Arnold Engineering \\
\hline Motor Laminations & Laser Technologies, Inc. \\
\hline Motor Winding & Windings, Inc. \\
\hline Motor Drive & Saminco, Inc. \\
\hline Thermal Analysis & UES, Inc. \\
\hline Composite Wheel & Universal Superabrasives, Spencer Composites \\
\hline Integration & Eaton MTC \\
\hline Program Mgmt. & Eaton MTC \\
\hline
\end{tabular}

As given previously, the overall spindle design was guided by the following general specifications:

- Spindle Speed, constant torque from 0 to 2,000 RPM, constant Horsepower (50) from 2,000 to 7,000 RPM

- Grinding Wheel Diameter, 12" to 14"; wheel width, 8" to 12"

- Through-feed and In-feed Capable

- Spindle Stiffness, 2,000,000 lbs/in (minimum)

- Small, Compact Packaging

A "process-driven design" approach was applied to develop the spindle concept. To minimize overall spindle size, the spindle arrangement incorporates an innovative "inside-out" brushless DC motor operating on "intelligent" tilting-pad hydrodynamic bearings. A direct drive motor is used in place of belts and pulleys to improve part quality by eliminating belt/pulley vibration sources. Ideally, by placing the motor integrally between the bearings, the spindle should be able to be dropped in into any twin-grip style centerless grinder. For this program, however, the new Viking centerless grinder was selected as the candidate grinder. The Viking was specifically designed with input from Eaton MTC so as to maximize the performance benefits arising from the use of superabrasives.

Obviously, precision component tolerances require high stiffness machines. The static loop stiffness of the Viking grinder was measured at 
$3,000,000 \mathrm{lb} / \mathrm{in}$ between spindle saddles and 1,200,000 lb/in directly between the current rolling element spindles (see Figure 1). Therefore, for this project, the total spindle stiffness was targeted at 2,000,000 lb/in. At high rotational speeds, damping is also critical to reduce the effects of vibration which can be directly translated to the workpiece surface, especially when grinding brittle materials such as ceramics. All of these requirements indicate that the use of fluid film bearings would be desirable.

Utilizing the above approach, the following statement of work was conducted in a simultaneous product development mode. There were five (5) major technical Tasks:

\section{Task 1. Spindle Design and Verification}

The spindle bearing selection and configuration for a centerless grinding machine spindle shall be designed and its performance verified through readily available analytical methods as may be deemed necessary and appropriate to meet minimum project goals.

\section{Task 2. Motor Development}

A motor capable of supplying the necessary speed and power to the grinding spindle shall be designed or selected. The motor shall be designed into the spindle housing such that an externally mounted motor will not required for spindle operation.

\section{Task 3. Wheel Hub Development}

A wheel hub, designed for easy installation of the grinding wheels and accurate rotational characteristics (e.g., low run-out and vibration) shall be designed.

\section{Task 4. Wheel Balancer Development}

Based on overall cost effectiveness, an automatic dynamic wheel balancing system shall either be designed or selected from available commercial models for use with the grinding spindle. If applicable, the balancer shall be integrated into the design of the overall spindle system.

\section{Task 5. Sensors, Sealing, and Support Hardware Development}

Sensors for monitoring spindle speed, vibration, and temperature shall be selected and incorporated into the design of the spindle. Methods for sealing parts of the machine from the harmful effects of fine grinding debris and coolant fluids shall be incorporated into the final design. Any necessary support hardware, such as chillers, filters, air supply, and fluid separators shall be designed or selected and integrated into the spindle-system design.

A discussion and presentation of the resulting design is given in the following section. 


\subsection{RESULTS and DISCUSSION}

\subsection{OVERALL SPINDLE CONFIGURATION}

Given the design objectives of this program, the major challenge was to develop a spindle concept which minimized overall size while providing a powerful self-contained package. Several alternative approaches were evaluated. Initially, conventional rotating shaft arrangements were considered. For example, the current Viking grinder (Figure 1) incorporates a rolling element grinding spindle which is externally driven through a long set of vee-belts. In this case the shaft which supports the grinding wheel rotates as a complete assembly inside several sets of angular contact bearings while the outer bearing races remain stationary. This type of arrangement could be driven directly by externally mounting a motor directly inline with the shaft (i.e. where the current spindle pulley is mounted). Although the approach is feasible, the overall width of the machine is increased by the width of the motor and is not consistent with the statement of work (see Task 2).

Subsequently, the concept of moving the motor inboard between the bearing saddles was evaluated. A preliminary design based on this type of layout is given in Figure 2. With this approach the width between bearing saddles must be increased beyond the wheel width by an amount equal to the motor width. Although the overall package will fit inside a Modler centerless grinder, it is too wide to "drop" into a Viking. It is also interesting to note that this design is based on a Modler spindle whereby the grinding wheel rotates about a stationary shaft (or axle). The setup is very analogous to the way in which a non-driven automobile wheel rotates about its stationary axle.

The inherent stiffness, size, and manufacturing benefits associated with the stationary shaft approach prompted the concepting of the design shown in Figure 3. Building upon this stationary shaft approach allows for an "inside-out" motor to be located directly inside of the grinding wheel hub. Although thermal concerns are obvious, the overall package itself is extremely compact and is generally noted for smooth operation. As an aside, it is interesting to note that inside-out motors are also commonly used for computer disk drives and muffin fans because of these inherent benefits. Recognize, however, that Figure 3 is merely a concept drawing showing only a single row of angular contact bearings. Additional bearing analysis, given in the following section, lead to the selection and design of a unique inside-out tilting-pad hydrodynamic bearing arrangement.

Based on this novel hydrodynamic bearing design and a stationary shaft, the solid model shown in Figure 4 was generated. This scale model is representative of the final design concept which will easily fit into a Viking centerless grinder. Mounting is accomplished by simply "dropping" the stationary shaft into the existing spindle bearing cradle of the Viking. As Figure 4 shows, the entire motor and bearing assembly is completely contained within the grinding wheel. In this case the grinding wheel is 9.5 " wide and 14 " in diameter. 
The brushless DC motor, described in subsequent sections, will produce 62.6 HP at 7,000 RPM. Resulting wheel speeds will be 25,656 SFM at 7,000 RPM and a maximum of 33,000 SFM at 9,000 RPM. To maintain thermal stability and avoid excessive heating the spindle motor also incorporates internal water cooling as described later.

To clearly understand this overall design it is worthwhile to look at how the "package" is assembled. Additional component details will be provided in the following sections. As indicated above, the spindle is based on a stationary shaft as shown in Figure 5. Subsequently, the DC motor lamination stack (stator) is positioned centrally on the shaft (see Fig. 6). Then, the two inside-out tilting-pad bearings are mounted adjacent to the stator as shown in Figure 7 . The assembly is then completed by adding the side-plates, rotor yoke, and wheel/hub assembly (see Fig. 8). The final spindle assembly drawing is given in the appendix as Drawing \#1. Although a considerable level of detail has been omitted in this "pseudo-assembly" process, the overall components and results are the same.

\section{2 - BEARING DESIGN AND PREDICTED PERFORMANCE}

The single most important decision in the design of any high performance spindle, involves the choice of bearing type, quantity, and placement. The results of this decision will directly establish the final spindle performance, cost, and overall reliability. Just as the spindle can be considered as the "heart" of the machine tool, the bearings are the "heart" of the spindle. Therefore, in order to make the appropriate bearing selection, numerous tradeoffs must be carefully evaluated and ranked based on critical priorities. A list of typical spindle bearings and their relative performance attributes are given in Table 1.

Considering the attributes summarized in Table 1, the need for speed (i.e. high DN value) is probably the number one driver in this NGS program. However, a bearing arrangement which is also compact (to fit within the grinding wheel envelope) and does not require much support equipment (to minimize overall machine foot print) is a close second in terms of priority. Accuracy, followed by cost, are probably at the next level of priorities. Based on these drivers, the "inside-out" tilting-pad hydrodynamic bearing was the top choice. It is important to make the distinction that with this type of "inside-out" bearing, the journal rotates around the outside of a stationary bearing. By positioning the journal around the bearing a considerable space savings results in addition to a reduction in the total number of components.

Also in close contention were the water-based hydrostatic bearings and the hybrid ceramic angular contact bearings. However, due to bearing space considerations and required support equipment the hydrostatic bearing was abandoned in lieu of the tilting-pad hydrodynamic approach. Recognize that the water-based hydrostatic bearings would take up more valuable space within the grinding wheel envelope and would also require a large hydraulic pumping system $(1000+\mathrm{PSI})$ which adds cost, maintenance, and valuable floor space. 
The hybrid ceramic angular contact bearings were also carefully considered for this application. However, due to their relatively low damping, it was felt that ceramic bearings may not be the best choice for grinding brittle materials. Beyond these alternatives, the oil-based hydrostatic bearings were abandoned due to their poor speed capabilities while the air bearings were rejected due to low stiffness and minimal crash resistance.

\subsubsection{Radial Bearing}

Once having selected a tilting-pad hydrodynamic bearing for this spindle both the normal grinding forces (i.e. radial loads) and thrust (i.e. axial) loads were approximated. Although the radial loads are many times greater than the thrust loads, the bearing set will be required to sustain both radial and thrust loads. For the radial loads, a worst case normal force of $2000 \mathrm{lbs}$ was used. This was based on a steady-state throughfeed rough grinding operation for silicon nitride using a 10" wide wheel, times a safety factor of 2. Maximum axial loads were based on one tenth of this level (i.e. $200 \mathrm{lbs}$. max.).

Given the design rationalization of the previous section, several concerns surfaced relative to using a non-conventional "inside-out" bearing arrangement. Based on previous experience in high-speed grinding, considerable radial growth of the grinding wheel and associated rotating components can occur from the "centrifugal forces". Therefore, as an initial design consideration, a simple deformation analysis of the various rotating components was performed to evaluate the effects of centrifugal body loads induced under the desired maximum spindle operating speed of 7,000 RPM. Figure 9 shows the radial growth associated with the ID of a disk representative of the hub side plates that are used to carry grinding loads from the wheel to the bearing. The ID of the disk corresponds to the bearing diameter of the journal bearing. For rotating stresses, deformation of the ID is proportional to the square of the operating speed. In this analysis, the radial growth can range from 0.017 " at 7,000 RPM to $0.035 "$ at 10,000 RPM.

Since the typical clearance and minimum film thickness for this type of journal bearing are on the order of 0.005 and 0.0003 inches, respectively, it can be seen that bore growth is a significant portion of the overall clearance and must be addressed accordingly. Two approaches were evaluated to deal with this radial growth. The first, was to eliminate the inside-out geometry of the initial design concept by using a "cantilevered hook" type of bearing as is shown in Figure 10. In this case, the centrifugally induced growth would cause the journal to grow into the bearing, increasing the preload and resultant stiffness of the bearing. However, finite element analyses (see Figures $11 \& 12$ ) of the journal under predicted pressure distributions and centrifugal forces illustrate that the bearing would be subjected to unacceptable cantilever deflection resulting in bearing misalignment. As will be discussed later, even slight misalignment results in severe reduction of load carrying capacity, eliminating this as a suitable option. 
Another approach would be to "move" one (or all) of the pads out radially in concert with the growth of the journal. After evaluating several concepts, it was decided to use a three pad arrangement where two of the pads would carry all of the rotor weight and grinding loads, and a third pad would be able to compensate for the growth (see Figure 13). Although 5 and 7 pad arrangements were also considered for potentially improved stability, the 3 pad configuration was selected since "three points always define a circle". Recognize that as the moveable (actuated) $3^{\text {rd }}$ pad changes position to compensate for bore growth, the resultant bearing configuration would still define a single diameter circle capable of matching the "new" journal diameter. Also, by moving only 1 pad, the two stationary (i.e. non-actuated) pads can directly transfer the grinding loads into the stationary shaft without any loss of stiffness.

After analysis of the load angles (rotor weight and grinding loads) it was shown that two stationary pads spaced 120 degrees apart could support the total load range. The only possible exception could occur during heavy dressing. Depending on the magnitude and direction of the dressing forces, it could be possible to unload the two stationary pads and load into the actuated pad resulting in reduced stiffness. To correct for this, a slight spindle clocking adjustment may be necessary to reposition the spindle during the dressing cycle if heavy dressing forces were developed. Alternatively, if high dressing loads were always present, the best approach might be to reposition the dresser such that the loads are directed into the stationary pads.

In addition to compensating for journal bore growth, this third pad also serves to apply a preload force on the other two bearings. Because the stiffness of a journal bearing is characteristic of both operating speed and applied load, the resulting bearing stiffness can be changed at any operating speed in realtime. This concept represents a significant "breakthrough" for spindle bearing technology since the overall stiffness can be adjusted to best suit any given grinding application.

As an interesting aside, the common machine-tool school of thought is that "stiffer is better". However, based on the results of an ACMT program funded by Oak Ridge, it was clearly shown that severely out-of-round brittle ceramic components will round-up better using a system with a greater degree of compliance. Imagine an adaptive control system which initially starts the rough centerless grinding process while operating at a reduced stiffness; then, as roundness improves, the bearing stiffness is increased to better control final part diameter. This novel idea has been coined as Controlled Compliance Grinding (CCG) by the authors. It is felt that by applying CCG technology to high-speed centerless grinding of ceramics, considerable quality and cost improvements will be realized.

The next step involved evaluating several methods for applying the actuated pad loads. One method, typically used in air bearing applications, involves supporting one pad by a soft spring (Belleville washer). In this case, the required total preload force (as detailed in the predicted bearing performance discussion) would result in an excessive startup toque under dry (no oil)

conditions. As a result, the operator would not be able to spin the wheel by hand 
to examine wheel condition, balance the wheel, or perform any other setup operation that is typically encountered. Also, bearing pad temperature and wear would be excessive at low spindle speeds. Other mechanical methods included the use of screws or ball-ramps to provide radial pad displacement. These proved to be too complex and unreliable. In addition, such systems would require nearly constant adjustment as the spindle speed or temperature changed. As a result, it was decided to actuate the pad hydraulically to compensate for dimensional bore changes. With this approach the spherical pivot becomes an actuated piston. Therefore, by applying constant hydraulic pressure, the bearing preload and clearance would also remain constant.

Given the above benefits, use of the hydraulically actuated pad concept allows the following enhancements over standard journal bearing approaches:

- Constant bearing clearance independent of induced journal/bearing differential growth (thermal or rotational)

- Allows starts \& stops with little or no preload

- Real-time controllability of spindle bearing stiffness (for process optimization)

Again, Figure 13 shows an actual photograph of the final bearing assembly. Detailed bearing drawings are also provided in the appendix as Drawing \#2 through Drawing \#8. As stated above, the radial grinding loads are supported by two stationary tilting pads while the third tilting pad is hydraulically actuated to preload the bearing, thus producing the desired overall bearing stiffness. Note that tilting pads were selected because the spindle can be run under a variety of loads and speeds with outstanding stability. Operation of the spindle can occur over a range of 1,250 to 10,000 RPM and from no load to heavy grinding loads. Allowing the pad to tilt enables the bearing to maintain a constant inlet/exit film thickness ratio as the pressure distribution varies from the changing grinding loads and speeds.

Based on a comprehensive bearing misalignment analysis (see Figure 14) it was critical that a "ball-and-socket" tilting pad support be used to allow for some degree of axial tilting or misalignment. Since this is a high-load bearing design (eccentricity ratio $>0.9$ ), adding an axial degree of freedom prevents reductions in load capacity that can result from even slight rotor misalignment. Recognize that for a slight misalignment of only $0.02^{\circ}$, the load carrying capacity can be reduced by more than half of that for a perfectly aligned journal (see Figure 14). With this "ball-and-socket" tilting pad support, however, minor pad misalignment becomes a non-issue (i.e. fully self-aligning).

Additional analysis of this journal bearing design included utilization of several computer-based hydrodynamic bearing codes; including Cojour, ROTNL, and Flimp. The results of these analyses, given in Table 2 and Figures $15-19$, show the predicted operating and performance characteristics for this journal bearing design. The target stiffness for the spindle of $2,000,000 \mathrm{lb} / \mathrm{in}$ is achieved by using two fluid film bearings, each with a stiffness of $1,000,000 \mathrm{lb} / \mathrm{in}$ (analogous to springs in parallel). Notice that to maintain a constant $1,000,000$ $\mathrm{lb} / \mathrm{in}$ bearing stiffness, the bearing preload must be increased with speed (see 
Figure 15). In this case, a hydraulically induced bearing preload of $836 \mathrm{lbs}$. is required at 7,000 RPM to achieve a bearing stiffness of $1,000,000 \mathrm{lb} / \mathrm{in}$.

To identify the sensitivity of the various bearing characteristics relative to overall stiffness, the bearings were also analyzed for stiffnesses of 500,000 and $1,500,000 \mathrm{lb} / \mathrm{in}$. It was found that sufficient load carrying capacity was attained using only $65^{\circ}$ arc pads such that sufficient room was available to incorporate a thrust bearing (described below) between each pad.

Figure 16 is a plot of minimum film thickness as a function of operating speed. In all cases the resulting film thickness is generously thick for a bearing of this diameter. Also notice that as operating speed increases, journal eccentricity increases, generating a thicker film. However, to have a stiffer bearing, the preload must be increased (Fig. 15) which tends to reduce film thickness. The minimum film thickness at the $1,000,000 \mathrm{lb} / \mathrm{in}$ requirement and 7,000 RPM appears to be around 0.0003 inches which is sufficiently thick.

The amount of power that a single bearing will consume through shearing of the fluid is presented in Figure 17. To minimize viscous drag and resultant heating a thin spindle oil, such as Shell Tellus 10 spindle oil (only 1 micro-reyn at $100^{\circ} \mathrm{F}$ ), has been selected for this application and was used in all analyses. Note that at 7,000 RPM, the two sets of journal bearings will consume a total of approximately 11 horsepower. Recognize that this parasitic power loss must be taken into consideration when designing the motor so that sufficient power (50 $\mathrm{hp}$ minimum) remains for productive grinding. It is interesting to note that the power loss is independent of stiffness and is only a function of speed. Similarly the required lubrication oil flow rate (as seen in Figure 18) is also independent of stiffness and only a function of speed. To provide adequate bearing cooling and lubrication, each bearing will need to be supplied approximately 2.5 gallons of oil per minute.

The peak film temperature as a function of operating speed is plotted in Figure 19. Under 7,000 RPM operation, the peak temperature should be below $250^{\circ} \mathrm{F}$. Notice that at higher stiffnesses (i.e. higher preloads) the bearing temperature is also somewhat higher. Recognize, however, that this is the peak temperature and not the bulk oil temperature. As such, overall the bearing will run cooler.

\subsubsection{Thrust Bearing}

Beginning with the three available arc lengths between radial pads (approx. $55^{\circ}$ between pads), a double acting thrust pad was incorporated into one bearing for use on one side of the spindle only. By placing both thrust pads in a "double-acting" configuration at one end of the spindle, the need to compensate for thermal growth along the entire length of the spindle can be eliminated. Such an approach is common practice in spindle design.

For this thrust bearing design exercise the target stiffness was set at $1,000,000 \mathrm{lb} / \mathrm{in}$ at $7,000 \mathrm{RPM}$ using only the preload of one thrust bearing acting against the other. The design was carried out using the well known Raimondi and Boyd (R\&B) thrust bearing design charts (circa 1955) to compute 
performance on a per-side basis. These charts are based upon a rectangular plane-slider pad configuration which is a good approximation for the actual sectored pad shape if the mean pad radius is used to calculate pad circumferential length and the difference between outer and inner radii is used as the pad cross length.

Preliminary analyses were done for the 7,000 RPM condition since flow requirements and power dissipation are highest for this maximum speed. Furthermore, the oil temperature will be highest at 7,000 RPM and thus the oil viscosity at its lowest. Therefore, at the lower speeds, the stiffness will not attenuate proportional to speed because the increase in lower-speed oil viscosity will compensate somewhat for this. Further analysis could be done to compute stiffness as a function of speed and thereby select the optimum total axial endplay (twice minimum film at zero applied load, 1.8 mills here).

At approximately 0.9 mills on a graph of thrust load versus film thickness, the slope of the curve gives $500,000 \mathrm{lb} / \mathrm{in}$ stiffness. Therefore, the double acting configuration with the corresponding preload would be $1,000,000 \mathrm{lb} / \mathrm{in}$.

Predicted total thrust bearing oil and power consumption will be $2.4 \mathrm{GPM}$ and 2.2 HP, respectively while oil temperature should only rise by $16^{\circ} \mathrm{F}$. Total lubrication requirements for both radial bearings and thrust bearings amounts to 7.4 GPM at $30 \mathrm{PSI}$. Also, the total parasitic power loss for all bearings is approximately 13 HP. Consequently, the integral motor should be designed for a total of $63 \mathrm{HP}$, thereby leaving $50 \mathrm{HP}$ available for productive grinding.

\subsection{MOTOR/DRIVE DESIGN and PREDICTED PERFORMANCE}

Most motors used in the world today are "induction" motors. The stator (stationary part of the motor) consists of wires wound around a steel core. When current is fed into the windings of the stator it generates an electromagnetic field. This field "induces" another electric field in the rotor (rotating part of the motor) which is made of "laminations" held together in an aluminum casting. The two fields repel each other and a rotating torque is generated. The larger the motor (or the longer the motor), the more torque is generated. Alternatively, the more power fed into the motor (voltage and current) the higher the torque generated.

Induction motors are common because the their construction is extremely rugged. The motors are maintenance free and the manufacturing processes have been refined so that the costs are very very low. Induction motors suffer from two problems, however. First, they have low efficiency - - - a lot of the input energy is wasted as heat and, secondly, it is difficult to control speed and position precisely.

DC motors, however, clearly address the efficiency and controllability issues. Typically, these motors use permanent magnets in either the rotor or the stator. In "brush type" permanent magnet DC motors, the stator field is generated by permanent magnets. The rotor field is generated by the current flowing through the windings in a laminated steel core. DC current is fed into the windings through carbon brushes that ride on the commutator. The permanent 
magnetic field makes brush motors more efficient than induction motors (since there is are no induction losses in the rotor). Also, since the permanent magnetic field is fixed, such motors are easier to control than induction motors. Changing the speed of a DC brush type motor is simply a matter of changing the input voltage - - - the speed of the motor is directly proportional to the applied voltage.

Another category of $\mathrm{DC}$ motors uses permanent magnets as well but the magnets are in the rotor. Current is fed into the stator and the rotating action is generated by power electronic switches. For this reason, this category of motors is also referred to at times as an "electronically commutated motor" or simply a Brushless DC Motor since there are no brushes. The advantages of brushless DC motors are numerous. Over induction motors the advantages include: much higher efficiency, much better controllability, lighter weight, faster response and higher speeds. There are a large number of variations in the types of motors - - (e.g. inside-out, pancake, etc.) each variation is based upon the manner in which the rotating electro-magnetic field is generated.

Switched reluctance motors were also a possible consideration for this NGS spindle. Typically, these motors have a "toothed" stator and rotor. Such motor designs are more efficient than an induction motor but less efficient than a permanent magnet brushless motor. However, it is also less expensive than a permanent magnet brushless motor since there are no magnets in the rotor. The disadvantage of these motors is that the it is much more difficult to design the motor since it has non-linear performance characteristics and manufacturing tolerances required are very tight.

\subsubsection{Motor Design \& Selection}

Given the above discussion, current state-of-the-art motorized spindle cartridges generally incorporate permanent magnet brushless DC motor technology. Although $A C$ variable frequency motors are also quite popular, the brushless DC motor arrangement was selected for this NGS spindle due to its power/size relationships and ease of integration into the overall spindle layout. As shown in Drawing \#9 in the Appendix, the 36 slot lamination stack is only 50 $\mathrm{mm}\left(1.97\right.$ ") wide with a diameter of $240 \mathrm{~mm}\left(9.45^{\prime \prime}\right)$ and will produce $62.6 \mathrm{HP}$ at 7,000 RPM! To generate such power density the motor incorporates costly rare earth samarium cobalt magnets in the 12 magnet poles which are embedded in the rotor yoke (see Drawing \#10). A maximum design speed of 9,000 RPM was utilized. The predicted motor performance is based on software utilized by MotorSoft (J. Hendershot) and gave the following results at 7,000 RPM:

- Power

- Torque

- Current Density

- Drive Current

- Efficiency

- Power Losses
62.6 HP

$464 \mathrm{lb}$-in

$3.48 \mathrm{amp} / \mathrm{mm}^{2}$

$67 \mathrm{amp}$

$97 \%$

1.7 HP total: 0.2 copper, 1.5 iron 
Recognize that this motor is essentially an "inside-out" design where the laminated and wound stator is on the inside while the solid rotor, which holds the permanent magnets, is on the outside (Drawing \#10). The hub (rotor yoke) is structural steel and the magnets are samarium cobalt. For this motor, the laminations are made from Arnon 7 (special) which have been annealed after stamping to reduce core losses. After stacking the laminations, the stator is wound using \#15 A.W.G. multi-strand conductor according to the specifications given in Figures 20 and 21. Using the MotorSoft motor simulation software, the NGS motor efficiency is predicted to be approximately $97 \%$. Losses occur through $I^{2} R$ power losses in the wire and from the generation of eddy currents in the stator iron. At 7,000 RPM operation, 1.7 HP will be lost of which 1.5 HP is through eddy currents in the iron and $0.198 \mathrm{HP}$ through $\mathrm{I}^{2} \mathrm{R}$ resistance in the copper wire.

Currently, most Brushless DC motors use the System Faulhaber® skew wound coil technology to provide low cogging. Typically, the lamination stack is skewed (or twisted) by approximately one slot along the axis of the stack. If the laminations were stacked perfectly straight across, the magnets would seek to center themselves over each lamination tooth. Thus, if the motor were turned by hand, a "click" could be felt as the magnet ratchets over to the next position. Pronounced cogging also prevents the motor from being "free spinning" with the motor off and puts extra strain on the drive at startup. By skewing the "lams", the magnet attraction is averaged out and there is no preferred equilibrium position. However, due to this inside-out motor design, it was decided to skew the magnets, rather that the lams, to simplify manufacture and assembly while achieving the same end result (see Drawing \#11 in the Appendix).

\subsubsection{Drive Design \& Selection}

Brushless motors have been in use for decades, mostly in high end industrial and military applications. Commutation in the servo versions is accomplished with Hall effect elements; while non-servo versions generally use counter-EMF. The main reason that brushless motors had not been widely used in high power industrial applications until recently was because of the limitations of silicon control technology. Until very recently, power transistors (MOSFETs) were just not good enough power switches to make high power commutation practical. The other reason is cost. Advances in semiconductor manufacturing have lowered the costs for these state-of-the-art MOSFETs. An efficient brushless motor controller requires at least six times as many MOSFETs (the best types are mandatory) as a typical brush-type motor controller for the same power level.

Much of this technology is now being put to use in full size electric vehicles. Recently, MotorSoft has been extremely active designing highperformance brushless DC motors for hybrid electric vehicles and high-speed milling spindles. To drive the MotorSoft Brushless DC motors, custom drive systems from Saminco Inc. have been employed. For this program, Saminco Inc. was called upon to design a drive for the NGS motor described above. The 
drive, a Saminco Model BR101-5, is capable of maintaining speed control to better than $0.1 \%$ error at full speed under zero to full load (7,000 RPM, 50+ HP). Speed control is maintained by feedback control of the counter-EMF (or back EMF) by ensuring that it falls within predicted levels before firing the next coil. Additional details are provided in the next section. As a backup, magnetorestrictive sensors will also be placed in the stator to sense the rotor poles passing by and to also give an indication of speed. The drive also has a dissipative braking system to quickly stop the wheel in emergency situations (e.g. E-Stop or other embedded sensors in machine to prevent crashes). A block diagram of the Saminco BR101-5 controller system is given in Drawing \#12. The brushless DC motor drive has the following general operating parameters:

Rating

Input

Output

Operational modes

Adjustable parameters

Protection
50kW@7,000 RPM continuous

3-Phase, 460 V AC, $60 \mathrm{~Hz}$

3-Phase, $0 . .460$ VAC (RMS), 0.100 A(RMS), $0 . .1000 \mathrm{~Hz}$ for $10,000 \mathrm{RPM} \max$ or $700 \mathrm{~Hz}$ for 7,000 RPM max.

Speed reference $\mathrm{P} 1$ control $0 . .10,000$ RPM Acceleration/deceleration: 0-200 RPM/sec Fail-safe emergency stop: Dynamic braking system during E-stop condition

Current limit Maximum speed Automatic phase advance parameter (reference speed, slope beta/speed, beta maximum) Against short-circuit, overloads, overspeed, system faults

On-board faults diagnostic

\subsubsection{Operation}

Control of the motor is achieved through two modes - startup and closedloop run. When the "run" signal is first applied to the drive, DC current is turned on in one set of motor windings to align the motor shaft. This signal remains on for a fixed duration of 0.15 seconds. After this time delay, a ramped stepped frequency signal from $1 \mathrm{~Hz}$ to $10 \mathrm{~Hz}$ is generated to initiate motor rotation. Ramp time is adjustable and is followed by a variable delay, typically 0.6 seconds (adjustable from 0.2 to 1 second) to allow settling from the speed-up ramp. After this delay, the drive switches to "closed-loop run mode."

Speed is set using a $0-10$ volt DC input signal that is proportional to the 0 to 7,000 (or 10,000) RPM operating range. The accel/decel module converts this voltage to a frequency reference signal " $\mathrm{ref}$ " and is applied to the commutator controller. Commutation is now provided from the closed loop controller using signals generated from the motor's back EMF. 


\subsubsection{Principle of BR101-5 Sensorless Commutation}

Once the motor is running at a speed greater than $1 \%$ of nominal (about $70 \mathrm{RPM}$ or $7 \mathrm{~Hz}$ ) there is sufficient back EMF to generate reliable square wave signals which can be applied to the commutator controller. The commutator controller consists of a VCO (Voltage Controlled Oscillator) capable of locking on to the motor frequency over a wide speed range, to generate symmetrical square wave pulses at $6 \mathrm{X}$ the motor frequency. These square wave pulses are applied to the appropriate "commutator" IGBT's which switch blocks of DC current to the motor's windings, in synchronization with the motor's angular velocity.

Once the motor is running, its back EMF contains large commutation notches, (as depicted next to "EMF Sensor" block 1), at each zero crossing and at 120 degrees after the zero crossing. These waveforms need to be cleaned up, and this is done in an active filter which receives blanking pulses from the "l Motor" sensing block, so that the output of the filter consists of pure sine waves as shown on the block diagram.

For centerless grinding this sine wave is converted to symmetrical square waves and then is eventually fed to a logic block which converts the 180 degree pulse to a 120 degree pulse, with its leading edge in time with the input leading edge (see waveforms on block diagram). The $120^{\circ}$ pulses are fed to a distributor to switch on IGBT dual gate driver modules and output gate drive signals turning on the IGBTs on for 120 electrical degrees, at $60^{\circ}$ intervals, in such a fashion that only two IGBTs are on at any given time. Speed and current control are achieved using a conventional inner-loop current regulator and PWM generator comprising function block whose output is supplied to a constant current regulator IGBT via a gate driver.

\subsubsection{Spindle/Motor Thermal Analysis}

Once having determined the operating characteristics of the brushless DC motor, a complete 3D model of the entire spindle assembly was made. The 3D model, shown in Figure 22, was used for both thermal and mechanical FEA analyses. Of particular concern was how the enclosed spindle/motor assembly would respond to the thermal losses from the motor. Although this rare earth brushless DC motor is extremely efficient $(97 \%)$ the previous calculations showed that approximately $1.7 \mathrm{HP}$ (1268 watts) must be dissipated as heat within the spindle structure. In addition to this, it was already shown that $13 \mathrm{HP}$ will be lost due to viscous drag within the bearings. Conveniently, the heat arising from the parasitic bearing losses will be carried off by the lubricating oil. On the other hand, the 1268 watts of $I 2 R$ heating must be directly addressed.

Two approaches were considered as potential means for addressing the ${ }^{2} \mathrm{R}$ heating. The easiest method is simply to allow the ambient cutting fluid to externally cool the entire spindle by convective "splash" cooling. More realistically, however, the second option involved the use of an internal motor cooling sleeve which employs forced-convective cooling via an ethylene glycol 
mixture. Given the enclosed nature of the spindle and the need for precise temperature control, the internal liquid cooling technique seemed the most plausible. As an interesting distant alternative, the use of a "heat pipe" was also discussed but abandoned in favor of the simpler cooling techniques.

Based on the 3D model shown in Figure 22, the UES Corporation conducted a transient thermal analysis using the ProCast software package. The maximum temperatures within the spindle were predicted for continuous operation at 7,000 RPM and 63 HP for both conditions described above. As expected, without any additional internal cooling the motor never reaches a steady state temperature condition. After 41 minutes the maximum temperature in the motor is predicted to be over $121^{\circ} \mathrm{C}\left(250^{\circ} \mathrm{F}\right)$ and still climbing toward thermal meltdown.

To address this problem an aluminum sleeve (depicted in Drawings \#1, 13,14 , and 15 in the Appendix) was designed to serve as an internal heat exchanger for the motor. Twenty-four parallel flow channels are located under the lamination stack. Heat is conducted from the motor stator into the sleeve where it is carried away by a room temperature ethylene glycol mixture passing through at roughly half a gallon per minute. Using this internal motor cooling sleeve, the UES software predicted that the spindle/motor assembly will achieve a steady state temperature distribution within 41 minutes. As shown in Figure 22 , the maximum temperature within this steady state distribution is approximately $66^{\circ} \mathrm{C}\left(151^{\circ} \mathrm{F}\right)$ after 41 minutes of continuous operation.

The lessons learned from this analysis are clear: Internal cooling is a must. Even though the brushless DC motor is extremely efficient, the spindle assembly will burn-up under full load conditions without any additional internal cooling. Also, since steady state thermal equilibrium is not reached for 41 minutes under full load, a spindle "warm-up" period is required for precision grinding. Based on this analysis, at least a one hour warm-up is anticipated; after which time the wheel should be precision dressed to the desired profile. To accelerate the warm-up period, the spindle should be operated at maximum speed using maximum bearing preloads. Ideally, the thermal profiles developed in production grinding should be duplicated during the warm-up period. This may require some preliminary grinding of scrap parts to generate the loads and heat expected during production. To better monitor and control thermal stability, the spindle will be equipped with numerous embedded thermocouples. Based on measured internal temperature data, any required warm-up cycles can then be optimized to achieve thermal profiles indicative of production. Such an approach will improve final accuracy and minimize dressing.

\subsection{ANCILLARY SPINDLE COMPONENT DESIGN CONSIDERATIONS}

The above discussions focused on the design of the primary spindle components (e.g. bearings and motor/drive system). In this section, design considerations for the stationary shaft, rotor yoke (hub), grinding wheel, side plates, and seals are addressed. In addition, a brief discussion relative to wheel 
balancing is also provided. Take note that the overall spindle design and thermal analysis presented in the previous section incorporated the spindle elements developed below.

\subsubsection{Shaft}

The central element in the NGS spindle is the stationary shaft (or axle) upon which all of the remaining spindle components are mounted. This steel shaft (3.75 inch OD approx.) is doubly-supported (i.e. held in a twin-grip configuration in the machine) and serves as the conduit for all fluids and electrical connections. The three power lines, bearing oil supply and drain lines, as well as the motor coolant supply \& drain lines are all drilled into the shaft and fed into the respective components. The porting arrangement and overall shaft configuration is given in Drawings \#15 and \#16. Sealing between the respective components and the shaft is accomplished using O-rings and gland seals as shown in the assembly drawing (Drawing \#1) and in Drawing \#19, respectively. To avoid leakage, the high pressure (3000 PSI) actuated pad hydraulic supply is plumbed though a separate line attached to the OD of the shaft.

\subsubsection{Hub-Wheel}

At high rotational speeds, the centrifugal body forces can become significant. Based on an FEA of the entire spindle package, it was shown that the heavy samarium cobalt magnets significantly increase the central deflection (radial growth) of the rotor yoke arising from centrifugal force. To add overall structural and thermal stability, a 1" thick graphite-epoxy composite sleeve was designed to be fitted over the motor rotor yoke assembly (see Drawing \#1). By utilizing recent advancements in grinding wheel technology, abrasive segments will be bonded onto this composite sleeve to form the final grinding wheel.

While inertia loads have an undesirable effect in terms of unloading the journal bearing, the same principle can be used to an engineering advantage when it comes to retaining the grinding wheel. The OD of the motor rotor yoke is a steel assembly. As indicated above, a graphite-epoxy composite sleeve was selected to serve as the grinding wheel hub. The composite structure can be manufactured to have a similar modulus as the steel hub, but at roughly one-fifth of the weight. The net result is that, under centrifugal force; the steel rotor yoke OD will grow more than the ID of the composite sleeve and is thereby constrained without the need for elaborate wheel clamps. Depending upon initial clearances, a "press fit" of up to 0.003 " radially can result at 7,000 RPM. By taking advantage of this Differential Centrifugal Expansion (DCE) a relatively simple wheel flange (see Drawing \#1) can be used to retain the wheel at high speeds.

Selection of the composite structure was reduced to a graphite-epoxy structure given the high strength and stiffness of the fiber. Utilizing a computer code solving the micromechanics equations representative of composite theory, 
various configurations or layups were analyzed. Two manufacturing methods were evaluated (layup vs. winding) to produce the following laminate structures:

1. Pure circumferential wrap $\left(0^{\circ}\right)$

2. Radially quasi-isotropic

3. Circumferentially quasi-isotropic

The radially isotropic layout would be fabricated by laying up sheets of prepreg at various angular orientations. A hollow disk would then be cut out of the layup. This method tends to generate excessive waste. While it does have very high radial strength, the stiffness (and hence deformation) in the axial direction under body loads is poor.

A filament winding approach could be used instead to generate a structure that is more cost effective and very strong in the hoop direction. Depending on the lead, the fiber could be wound in a nearly pure circumferential direction $\left(0^{\circ}\right)$ or achieve a quasi-isotropic structure in the hoop and axial directions. Based on the FEA studies conducted, the quasi-isotropic structure in the hoop and axial directions would be preferred since the centrifugally induced deflections from the magnets tend to cause bowing axially along the wheel width. Depending upon desired manufacturing cost and performance tradeoffs, the winding lead angle could range from $10^{\circ}$ to $45^{\circ}$.

\subsubsection{Side Plates}

The side plates (as shown in Drawings \#1 and \#18) are required transmit the grinding wheel loads into the bearings as well as carry the rotor hub and magnets. Ideally, the side plates should be of a single piece construction to eliminate potential interface separations arising from the centrifugal body forces. Initially, multi-piece side plates were designed to facilitate manufacture and ease assembly. Numerous bolted and slotted joint assemblies were analyzed using FEA methods. As shown in Figure 23, a bolted dovetail multi-piece assembly will undergo considerable joint separation from the centrifugal body loads resulting in reduced spindle stiffness and accuracy. Consequently, the design given in Drawing \#18 was ultimately selected. Although, either steel or composite side plates could be used, steel was selected for manufacturing simplicity. The resulting stresses and associated growth arising from centrifugal body loads are summarized in Table 3.

\subsubsection{Seals}

The most challenging and critical seal application in this NGS spindle design occurs between stationary bearings and the rotating side plates. In the selection and design of these seals numerous factors were considered such as leakage rate, cost, life, size, assembly, drag, speed and temperature limitations, etc.. After considering all of these factors in concert, it was felt that minimizing leakage had to be the number one priority. Although high performance labyrinth 
type seals were initially considered because of their high speed, long life, and low drag characteristics, in the final analysis they were rejected due to excessive leakage. It is critical to recognize that any leakage past the bearings into the internal motor cavity could lead to a severe imbalance condition as well as a possible short circuit of the stator windings. In addition, it was felt that zero leakage seals are also required to minimize tramp oil contamination of the grinding fluids as well as keep fine grinding swarf from entering the spindle itself.

To address all of these needs, a special high speed lip seal was developed in cooperation with American Variseal. This unique lip seal uses a Teflon-based fluoropolymer (Turcon 577) which can run at service temperatures up to $577^{\circ} \mathrm{F}$ and operating surface speeds as high as 12,000 SFM. When mated against a polished steel surface the coefficient of friction $(\mu)$ is less than 0.04 in the unlubricated state. Of course the life is proportional to PSI loading, which under the projected levels, should be more than adequate. A crosssection of the required seals are shown in Drawings \#19 and \#1.

Additional seals are required for the actuator (gland type) and the cooling sleeve (additional gland seals and ' $O$ ' rings) to ensure closed loop performance of all fluid circuits. Again, seals by American Variseal were selected. Turcon 99 gland seals were chosen for the actuator piston seals as well as the shaft-tobearing seals.

\subsubsection{Balancing}

Various balancing options were evaluated including servo-positionable weights; fluid-type, electromagnetic type and manual balancing. Recognizing the relatively high RPM operating requirements and very tight balancing specification (better than 0.2 micron peak-to-peak displacement) the servo weight method commonly found on conventional grinders could not be used. Two fluid-type system vendors were contacted and reported that their systems could probably be adapted but would require a significant amount of engineering (over $\$ 100 \mathrm{~K}$ ). The electromagnetic type had the response time and throw-weight required, however, being a relatively new product on the market, was cost prohibitive to integrate into the NGS spindle design.

Consequently, given the time and budget constraints of this contract, it was felt that the best option was to utilize a manual two-plane balance during the initial setup of the wheel and to monitor vibration levels during operation. Since this spindle is specifically designed for superabrasive grinding wheels the projected level of imbalance should be minimal after the initial two-plane balance is performed. Unlike a conventional wheel, which is subject to nonhomogenaity and large diameter changes over its useful life, the thin superabrasive wheel should remain relatively constant. As is conventional practice, however, any wheel should always be "spun dry" after each use to avoid localized imbalances arising from fluid residue. 


\subsection{SUMMARY}

Through efforts initiated by Oak Ridge National Laboratory, it has been demonstrated that high-speed grinding principles can be utilized in grinding silicon nitride with considerable improvements in throughput, costs, and quality. To take full advantage of this new of "High-Speed, Low-Damage (HSLD)" grinding process, however, centerless grinding machines will require high-speed, high-stiffness spindles. To accelerate the development of this new spindle technology, the Next Generation Spindle (NGS) program is focused on designing a direct drive $50 \mathrm{hp}$, twin-grip, centerless grinding spindle capable of using 1214 " diameter by 8-12" wide diamond grinding wheels with at least 7,000 rpm capability. Spindle stiffness is to be $2,000,000 \mathrm{lb} / \mathrm{in}$ minimum.

The overall design philosophy behind this effort is to use small diameter diamond wheels operating at high speeds. Such an approach will reduce wheel inventory costs and machine floor space requirements while minimizing the downside consequences of an in-process wheel crash. It is anticipated that major advances in cost effective ceramic finishing will result from centerless grinding at high material removal rates while utilizing the HSLD grinding process. Implementation of the NGS Spindle will also improve production flexibility by reducing the total number of centerless grinding machines (and corresponding setups) required for ceramic finishing operations.

Given the above approach, a novel fluid film spindle bearing arrangement, as well as an integral spindle/motor package, was designed. This new compact spindle incorporates a water cooled $63 \mathrm{HP}$ "inside-out" brushless DC motor operating on "intelligent" tilting-pad hydrodynamic bearings all within the small grinding wheel envelope. The overall design is based on a stationary shaft (or axle) about which the grinding wheel rotates - - - similar to the way a non-driven automobile wheel rotates about its stationary axle. Aside from the inherent stiffness, size, and manufacturing benefits associated with the stationary shaft approach, the design also allows for an "inside-out" motor to be located inside of the grinding wheel hub thus providing an extremely compact, direct drive system. The motor is referred to as an "inside-out" design since the laminated and wound stator is on the inside while the solid rotor, which carries the samarium cobalt magnets, is on the outside.

After evaluating several bearing concepts, it was also decided to design an inside-out, tilting-pad hydrodynamic bearing with 3 total pads. The benefits of the tilting-pad bearing approach include good high-speed capability, outstanding dynamic stability, compact size, minimal external system support, acceptable stiffness, and good crash resistance. With this novel inside-out design, the journal rotates around the outside of the stationary bearing. All of the rotor weight and grinding loads are supported by two of the pads while the third pad can be hydraulically actuated for intelligent control. The intelligent bearings can compensate for dimensional spindle variations as well as modifying bearing stiffness and damping in real-time. 
In ceramic grinding, "stiffer" is not always better. Based on the results of a pervious ACMT program funded by Oak Ridge, it was clearly shown that severely out-of-round brittle ceramic components will round-up better using a system with a greater degree of compliance. The intelligent bearings developed in this NGS program will allow for an adaptive control system which initially starts the rough centerless grinding process at a reduced stiffness; then, as roundness improves, the bearing stiffness is increased to better control final part diameter. The concept has been coined as Controlled Compliance Grinding (CCG) by the authors.

Full consideration was also given to high speed wheel design, mounting, and safety characteristics. At high rotational speeds, the centrifugal body forces can become significant. Based on an FEA of the entire spindle package, it was shown that the heavy samarium cobalt magnets significantly increase the central deflection (radial growth) of the steel rotor yoke arising from centrifugal force. To add overall structural stability, a 1" thick graphite-epoxy composite sleeve was designed to be fitted over the motor rotor yoke assembly. By utilizing recent advancements in grinding wheel technology, abrasive segments can then be bonded onto this composite sleeve to form the final grinding wheel. During highspeed operation, the steel rotor yoke OD will "grow" more than the ID of the composite wheel causing a "press-fit" between the two components. By taking advantage of this DCE (Differential Centrifugal Expansion) the wheel can be fully constrained at high speeds without the need for elaborate flanges or clamps.

After completing the entire spindle design, an overall thermal stability analysis was performed. The lessons learned from this analysis are clear: Internal motor cooling is a must. Even though the brushless DC motor is over $97 \%$ efficient, the spindle assembly could burn-up under full load conditions if internal cooling is not provided. To address this problem an aluminum sleeve was designed to serve as an internal heat exchanger for the motor. Twenty-four parallel flow channels are located under the lamination stack. Using internal motor cooling, a maximum steady state temperature of $151^{\circ} \mathrm{F}$ is reached after 41 minutes of operation. Consequently, a proper spindle "warm-up" period is required for precision grinding. Ideally, the thermal profiles developed in production grinding should be duplicated during the warm-up period. Following proper warm-up, the grinding wheel should be precision dressed at operating speed. To monitor and control thermal stability, the spindle will be equipped with numerous embedded thermocouples. Then, based on measured internal temperature data, the warm-up cycles can be optimized to improve final accuracy and minimize dressing. 


\subsection{ACKNOWLEDGMENTS}

Research sponsored by the U.S. Department of Energy, Assistant Secretary for Energy Efficiency and Renewable Energy, Office of Transportation Technologies, as part of the Ceramic Technology Project of the Materials Development Program, under contract DE-AC05-84OR21400 with Lockheed Martin Energy Systems, Inc.

In addition, the authors would like to acknowledge the efforts of the following key personnel:

\section{Eaton Manufacturing Technologies Center -} John Brennan, Sr. Design Engineer John M. Gorse, Sr. Laboratory Technician

Michael R. Cooney, Sr. Laboratory Technician

Case Western Reserve University -

Dr. Maurice L. Adams, Hydrodynamic Bearing Analysis

MotorSoft Corp. -

Mr. James R. Hendershot, Motor Design

Saminco, Inc. -

Mr. Bonne Posma, Motor Drive Design

Oak Ridge National Laboratory -

Dr. Peter J. Blau, Technical Concurrence 
Table 1 Spindle Bearing Performance Attribute Comparisons

\begin{tabular}{|c|c|c|c|c|c|c|}
\hline & $\begin{array}{l}\text { Steel - } \\
\text { Rolling }\end{array}$ & $\begin{array}{l}\text { Hybrid } \\
\text { Ceramic }\end{array}$ & $\begin{array}{l}\text { Oil } \\
\text { Hydro- } \\
\text { Static } \\
\end{array}$ & $\begin{array}{l}\text { Water } \\
\text { Hydro- } \\
\text { Static } \\
\end{array}$ & $\begin{array}{c}\text { Oil } \\
\text { Hydro- } \\
\text { Dynamic }\end{array}$ & Air \\
\hline Cost & Low & Medium & High & High & Medium & Medium \\
\hline $\begin{array}{c}\text { Crash } \\
\text { Resistance }\end{array}$ & Medium & Medium & High & High & High & Low \\
\hline Manufacturability & Low & Low & Medium & High & Medium & High \\
\hline $\begin{array}{l}\text { Required } \\
\text { Support } \\
\text { Equipment }\end{array}$ & Low & Low & High & High & Medium & Medium \\
\hline Maintenance & Low & Low & Medium & Medium & Medium & High \\
\hline $\begin{array}{c}\text { Temperature } \\
\text { Rise }\end{array}$ & Medium & Medium & High & Low & High & Low \\
\hline $\begin{array}{l}\text { Accuracy } \\
\text { (microns) }\end{array}$ & 1.0 & 1.0 & 0.1 & 0.1 & 0.5 & 0.1 \\
\hline $\begin{array}{c}\text { Speed - DN } \\
\left(\times 10^{6}\right)\end{array}$ & 1.0 & 2.0 & 0.5 & 2.0 & 2.0 & 0.2 \\
\hline Stiffness & Medium & Medium & High & High & Medium & Low \\
\hline Damping & Low & Low & High & High & Medium & Medium \\
\hline
\end{tabular}


Table 2. Bearing predicted performance.

\begin{tabular}{|c|c|c|c|c|c|}
\hline $\begin{array}{l}\text { Speed } \\
\text { (RPM) }\end{array}$ & $\begin{array}{l}\text { Preload } \\
\text { (lb) }\end{array}$ & $\begin{array}{l}\text { Minimum } \\
\text { Film } \\
\text { Thickness } \\
\text { (inch) }\end{array}$ & $\begin{array}{l}\text { Power } \\
\text { Loss } \\
\text { (HP) }\end{array}$ & $\begin{array}{c}\text { Flow } \\
\text { Through } \\
\text { Bearing } \\
\text { (gal/min) }\end{array}$ & $\begin{array}{c}\text { Peak Oil } \\
\text { Temperature } \\
\text { Rise } \\
(\circ)\end{array}$ \\
\hline \multicolumn{6}{|c|}{ Stiffness $=500,000 \mathrm{lb} / \mathrm{in}$} \\
\hline 2500 & 365 & 0.000374 & 0.99 & 0.81 & 185 \\
\hline 4000 & 450 & 0.000400 & 2.13 & 1.32 & 202 \\
\hline 5500 & 500 & 0.000447 & 3.57 & 1.86 & 211 \\
\hline 7000 & 600 & 0.000419 & 5.34 & 2.40 & 233 \\
\hline \multicolumn{6}{|c|}{ Stiffness $=\dot{1}, 000,000 \mathrm{lb} / \mathrm{in}$} \\
\hline 1,250 & 465 & 0.000228 & 0.33 & 0.36 & 187 \\
\hline 2,500 & 573 & 0.000280 & 1.02 & 0.78 & 207 \\
\hline 4,000 & 678 & 0.000305 & 2.19 & 1.29 & 225 \\
\hline 5,500 & 728 & 0.000320 & 3.63 & 1.83 & 238 \\
\hline 7,000 & 836 & 0.000320 & 5.43 & 2.37 & 258 \\
\hline \multicolumn{6}{|c|}{ Stiffness $=1,500,000 \mathrm{lb} / \mathrm{in}$} \\
\hline 2500 & 775 & 0.000222 & 1.02 & 0.78 & 224 \\
\hline 4000 & 860 & 0.000260 & 2.19 & 1.29 & 241 \\
\hline 5500 & 960 & 0.000266 & 3.66 & 1.80 & 259 \\
\hline 7000 & 1000 & 0.000277 & 5.49 & 2.34 & 274 \\
\hline
\end{tabular}

Table 3. Effect of centrifugal loads on side plate/journal.

\begin{tabular}{|r|c|r|r|r|}
\hline $\begin{array}{c}\text { Rotating } \\
\text { Speed } \\
\text { (RPM) }\end{array}$ & $\begin{array}{c}\text { ID } \\
\text { Change } \\
\text { (in) }\end{array}$ & $\begin{array}{c}\text { OD } \\
\text { Change } \\
\text { (in) }\end{array}$ & $\begin{array}{c}\text { Maximum } \\
\text { Hoop stress } \\
\text { (PSI) }\end{array}$ & $\begin{array}{c}\text { Maximum } \\
\text { Radial Stress } \\
\text { (PSI) }\end{array}$ \\
\hline 1,000 & 0.0000 & 0.0000 & 318 & 39 \\
\hline 2,000 & 0.0001 & 0.0001 & 1,270 & 157 \\
\hline 3,000 & 0.0003 & 0.0003 & 2,858 & 353 \\
\hline 4,000 & 0.0006 & 0.0005 & 5,081 & 628 \\
\hline 5,000 & 0.0009 & 0.0008 & 7,939 & 982 \\
\hline 6,000 & 0.0013 & 0.0011 & 11,432 & 1,414 \\
\hline 7,000 & 0.0017 & 0.0015 & 15,560 & 1,924 \\
\hline 8,000 & 0.0022 & 0.0020 & 20,323 & 2,513 \\
\hline 9,000 & 0.0028 & 0.0025 & 25,721 & 3,181 \\
\hline 10,000 & 0.0035 & 0.0031 & 31,754 & 3,927 \\
\hline
\end{tabular}




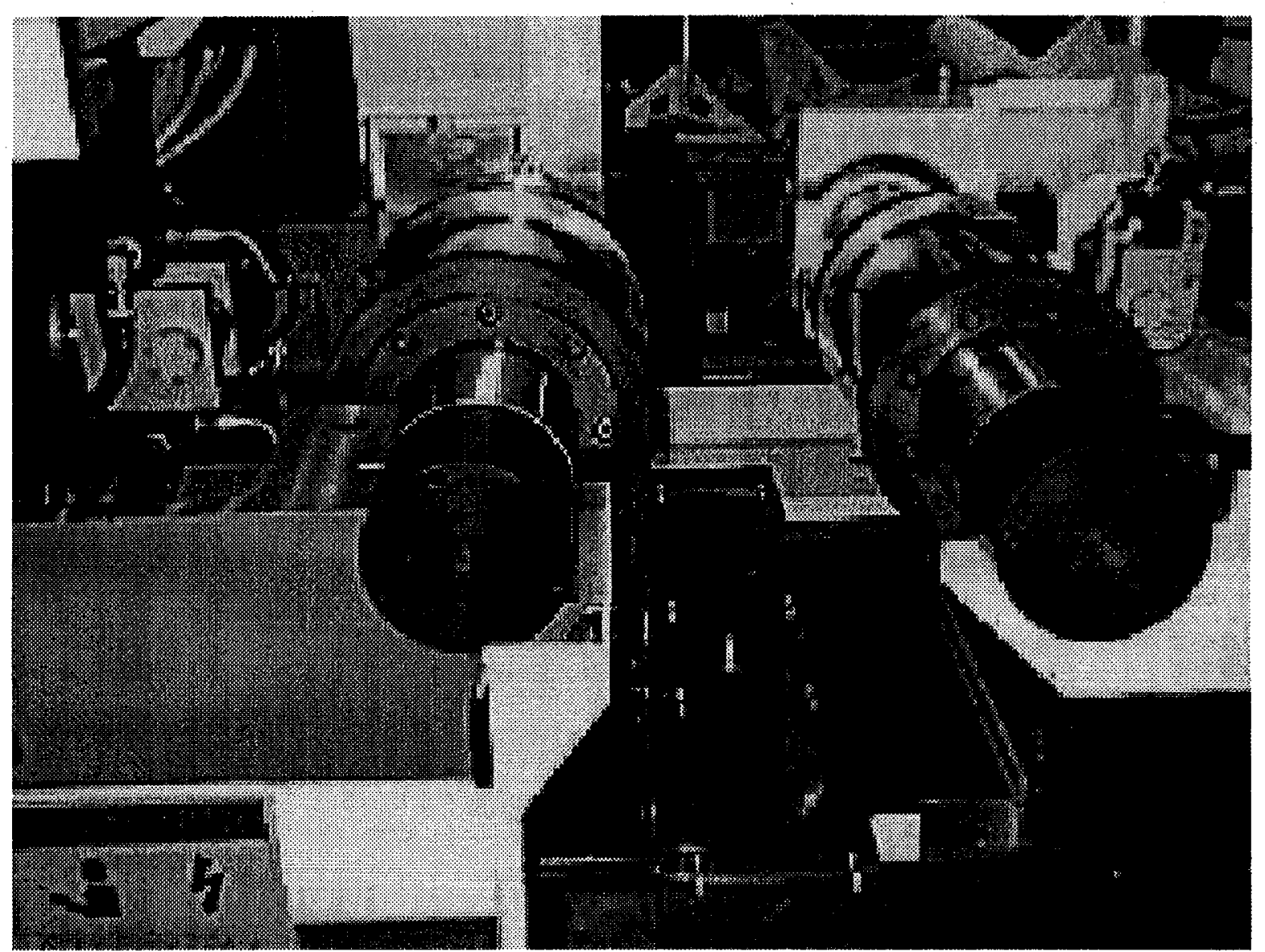

Figure 1. Viking centerless grinder - "Twin-grip" spindle arrangement. 


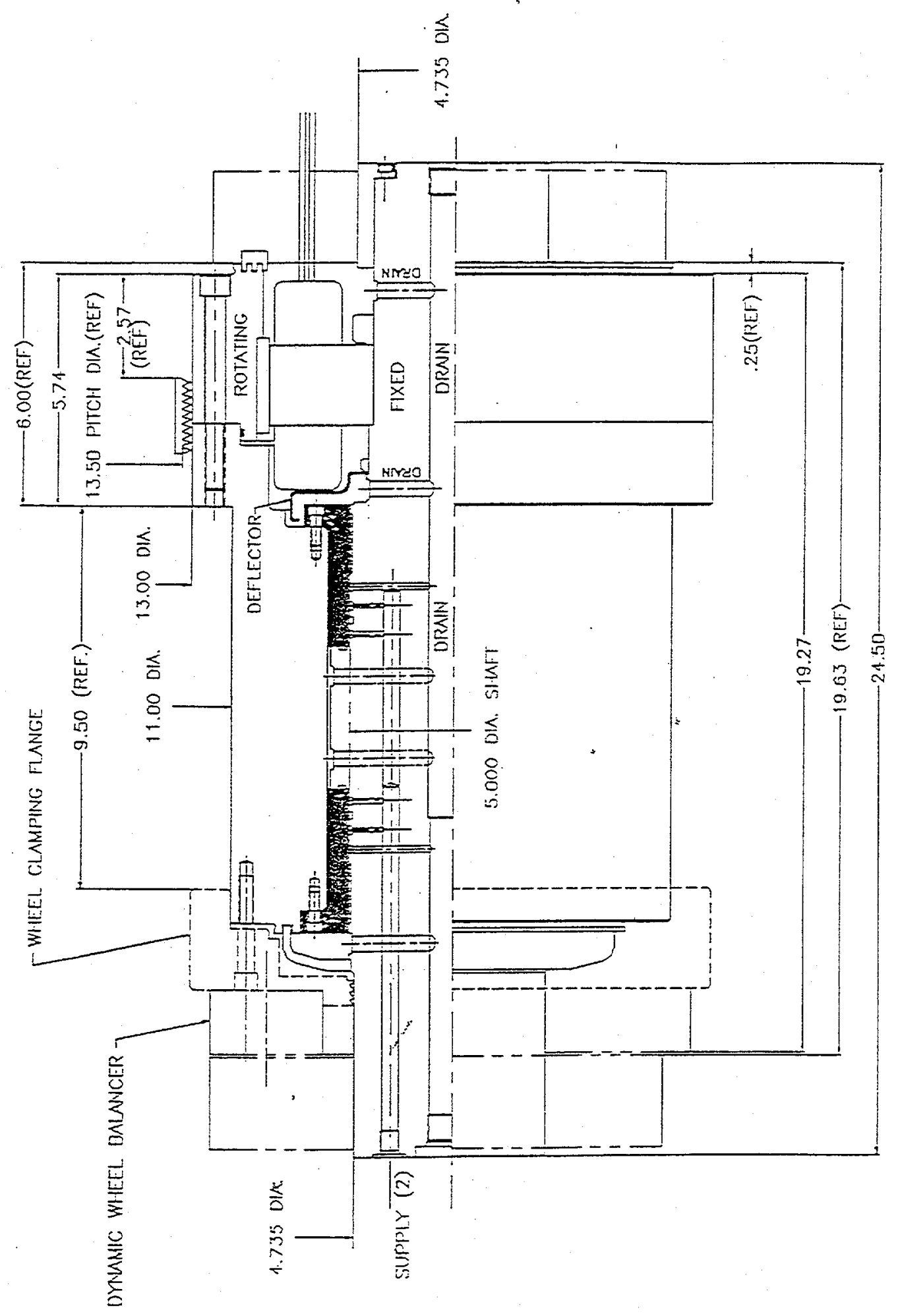

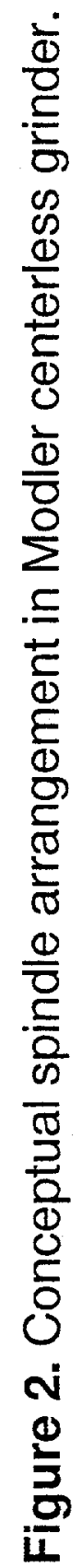




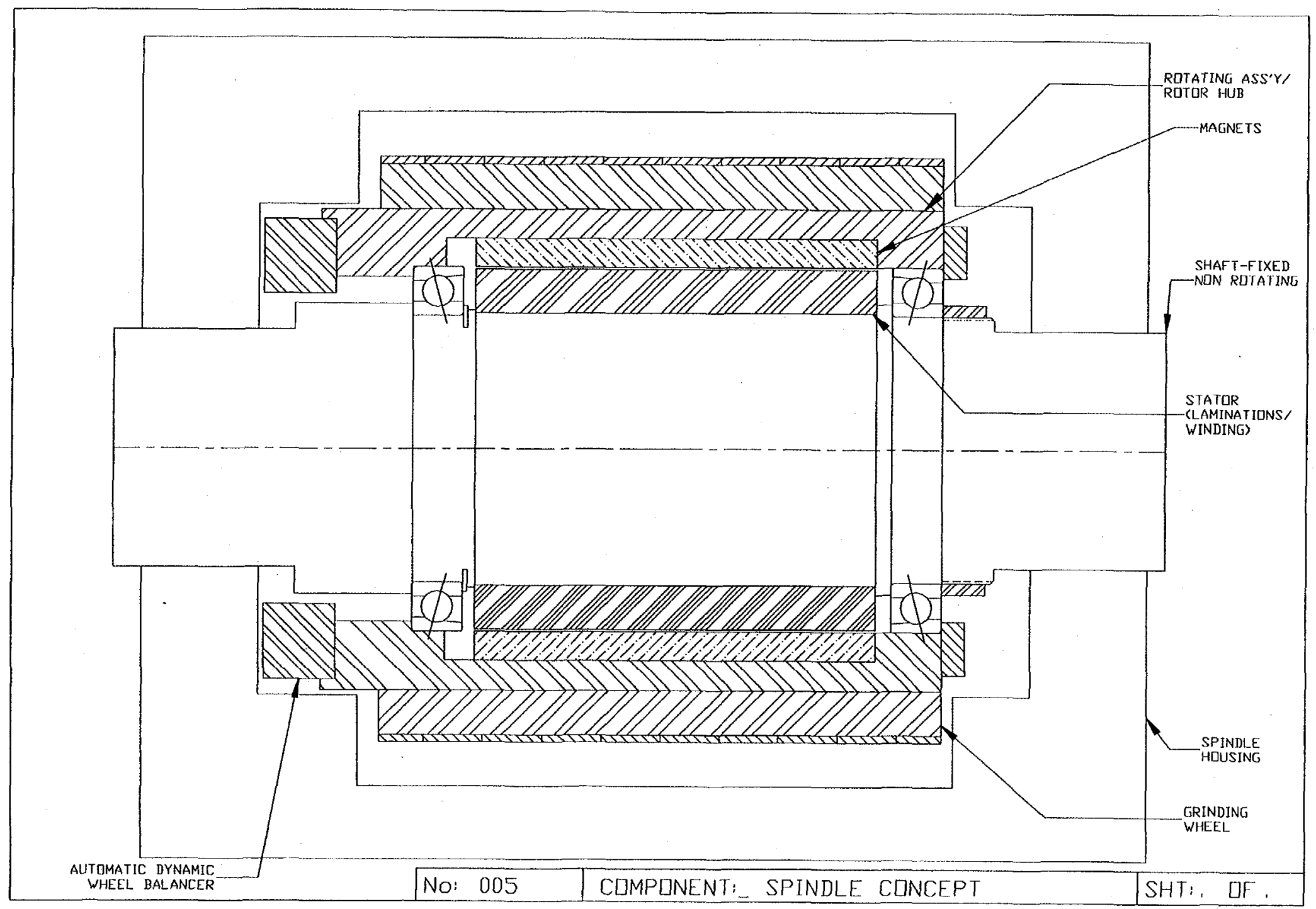

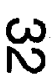

Figure 3. Spindle concept. 


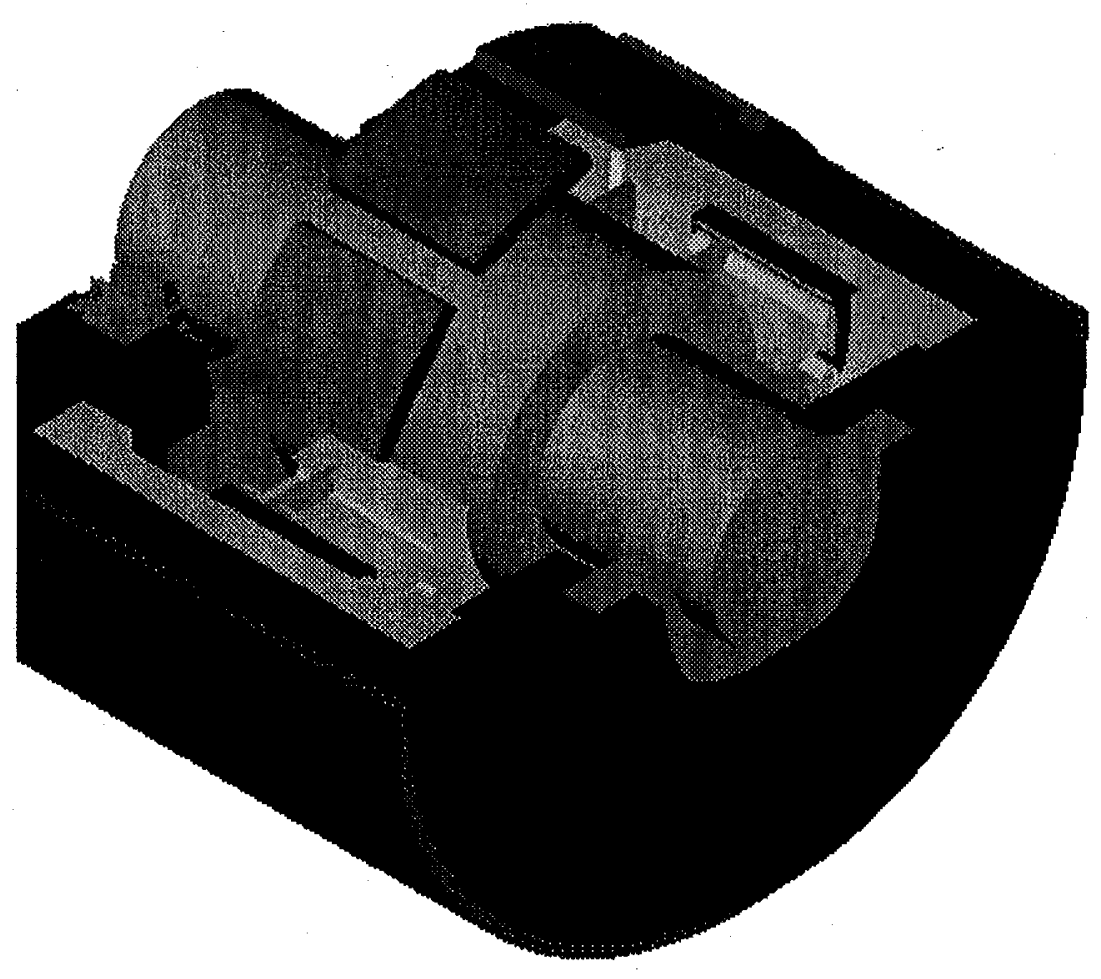

Figure 4. Next Generation Spindle - Overall conceptual model. 


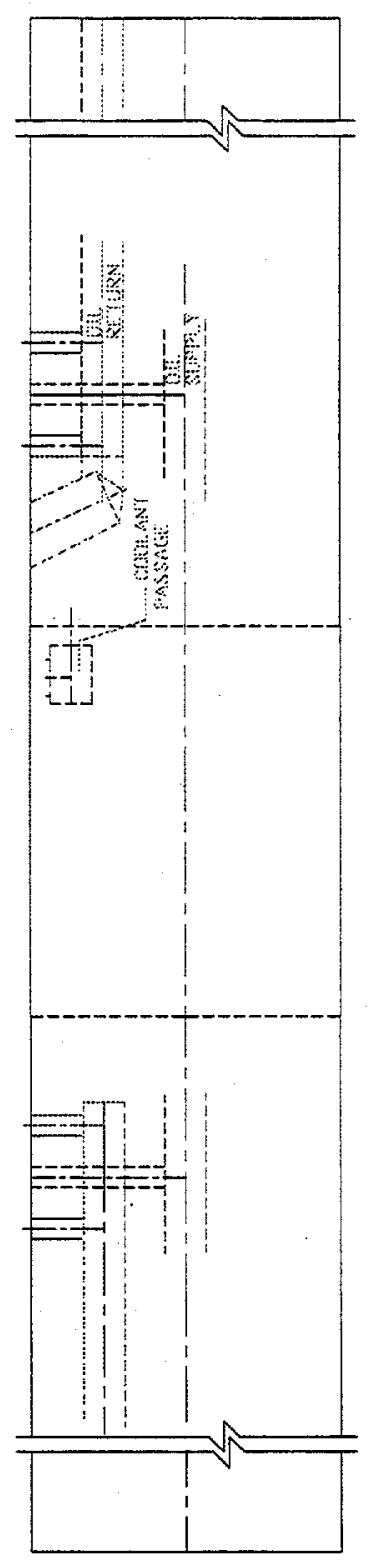

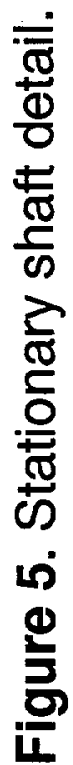



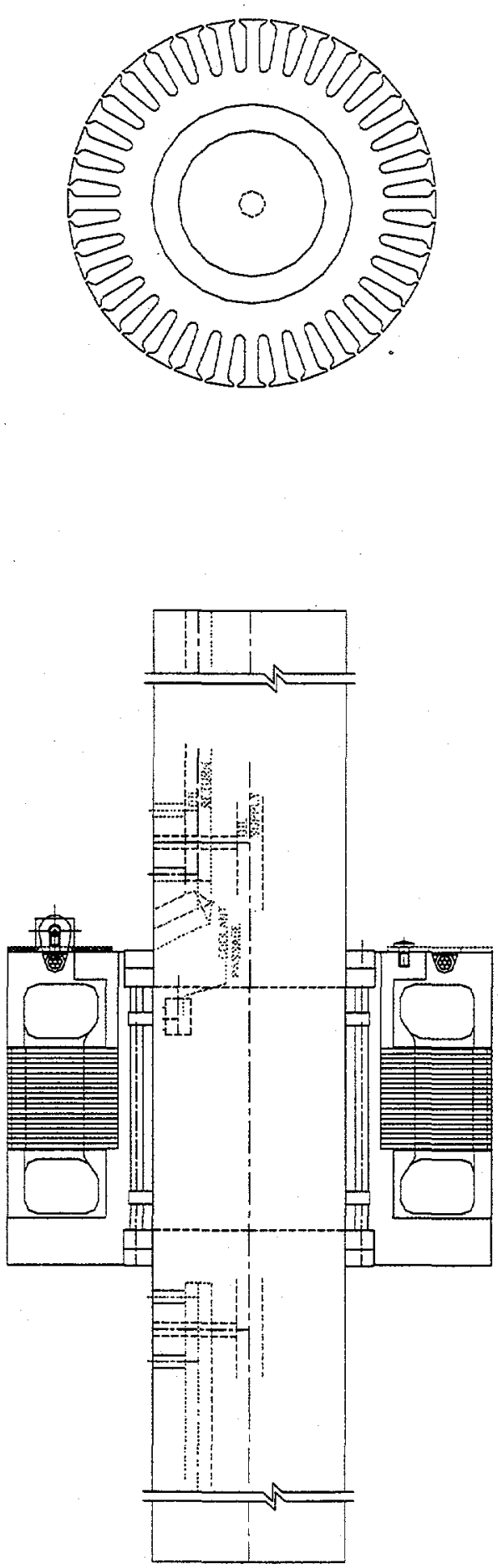

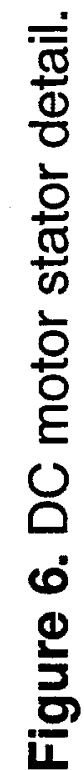



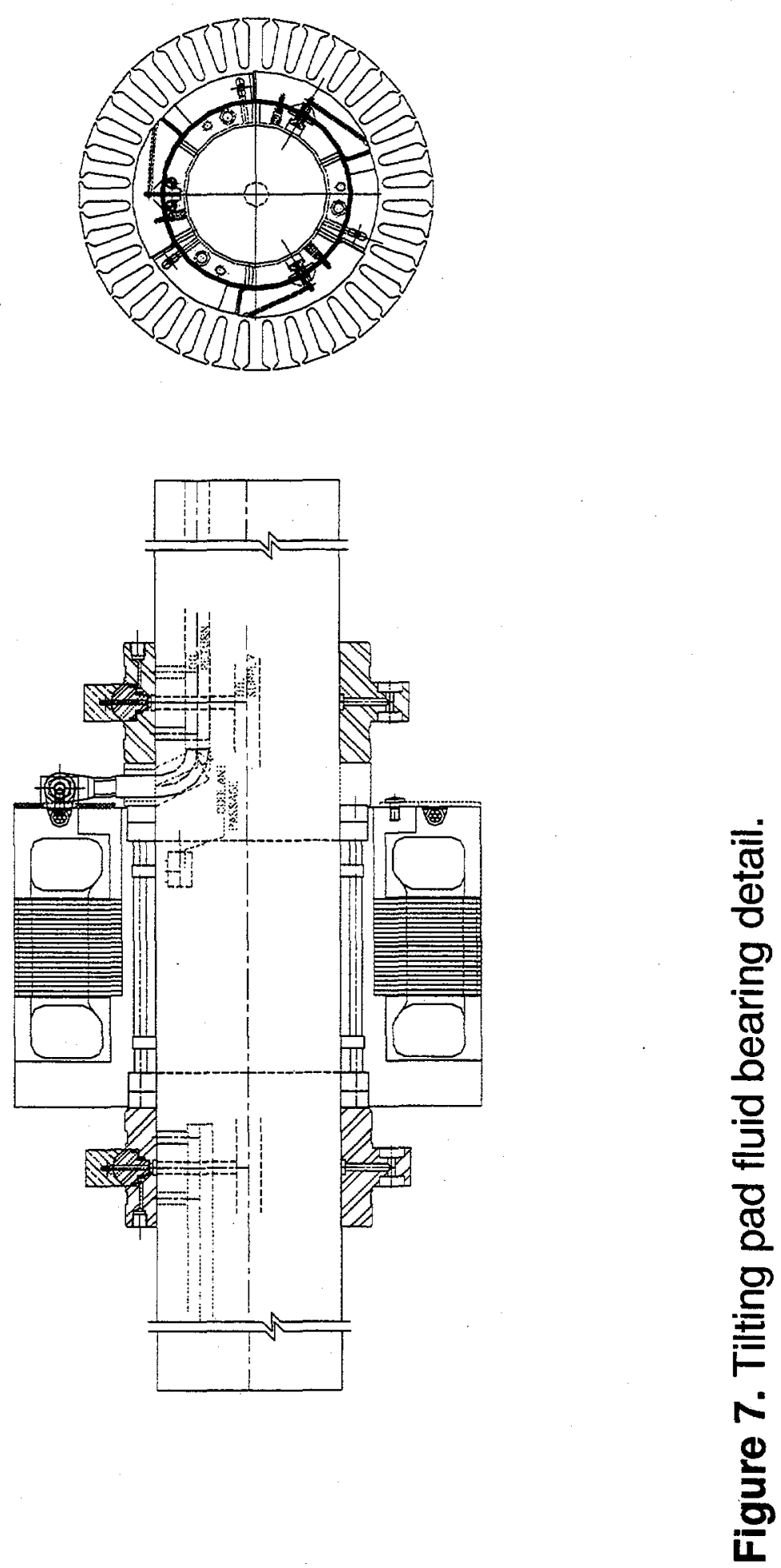

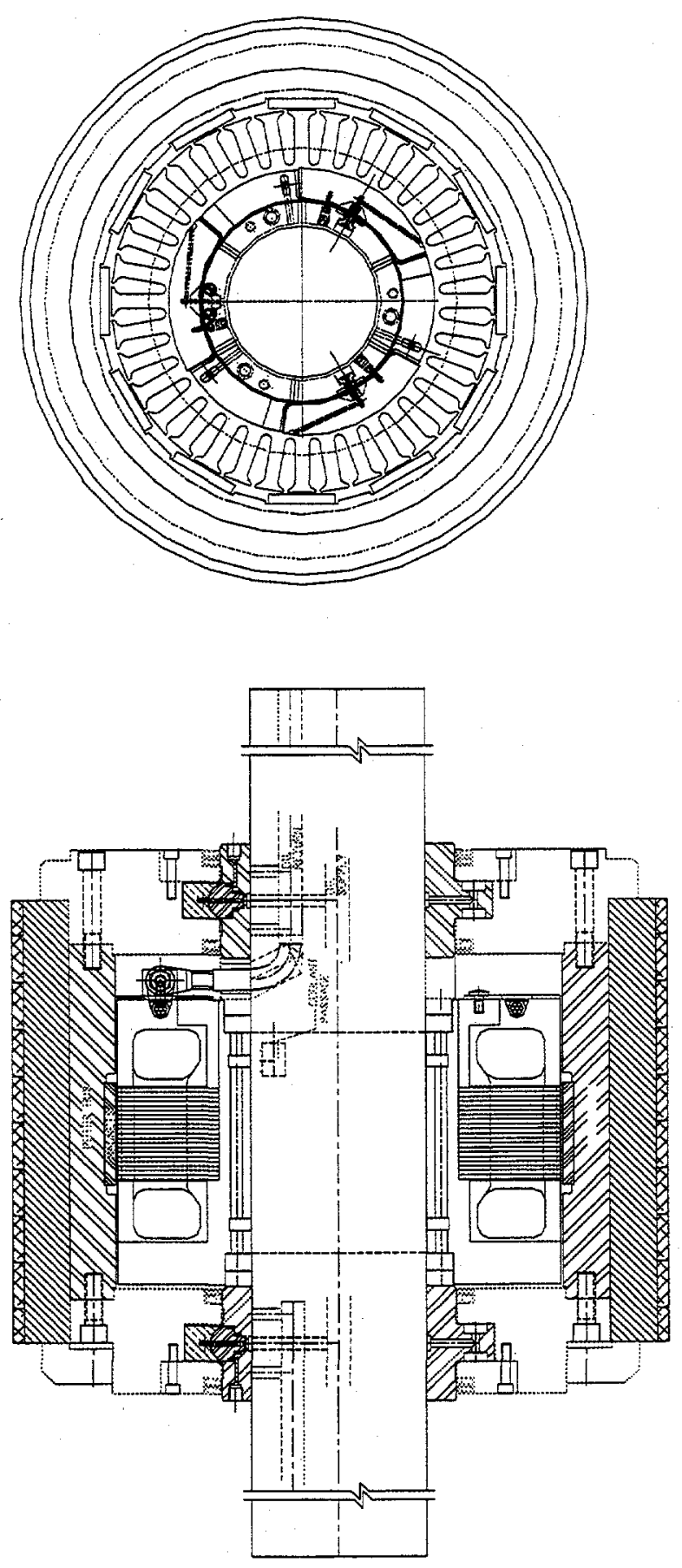

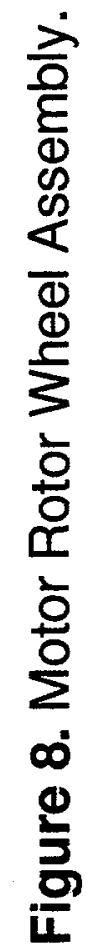


Bearing Journal Radial Expansion Due to Centrifugal Loads

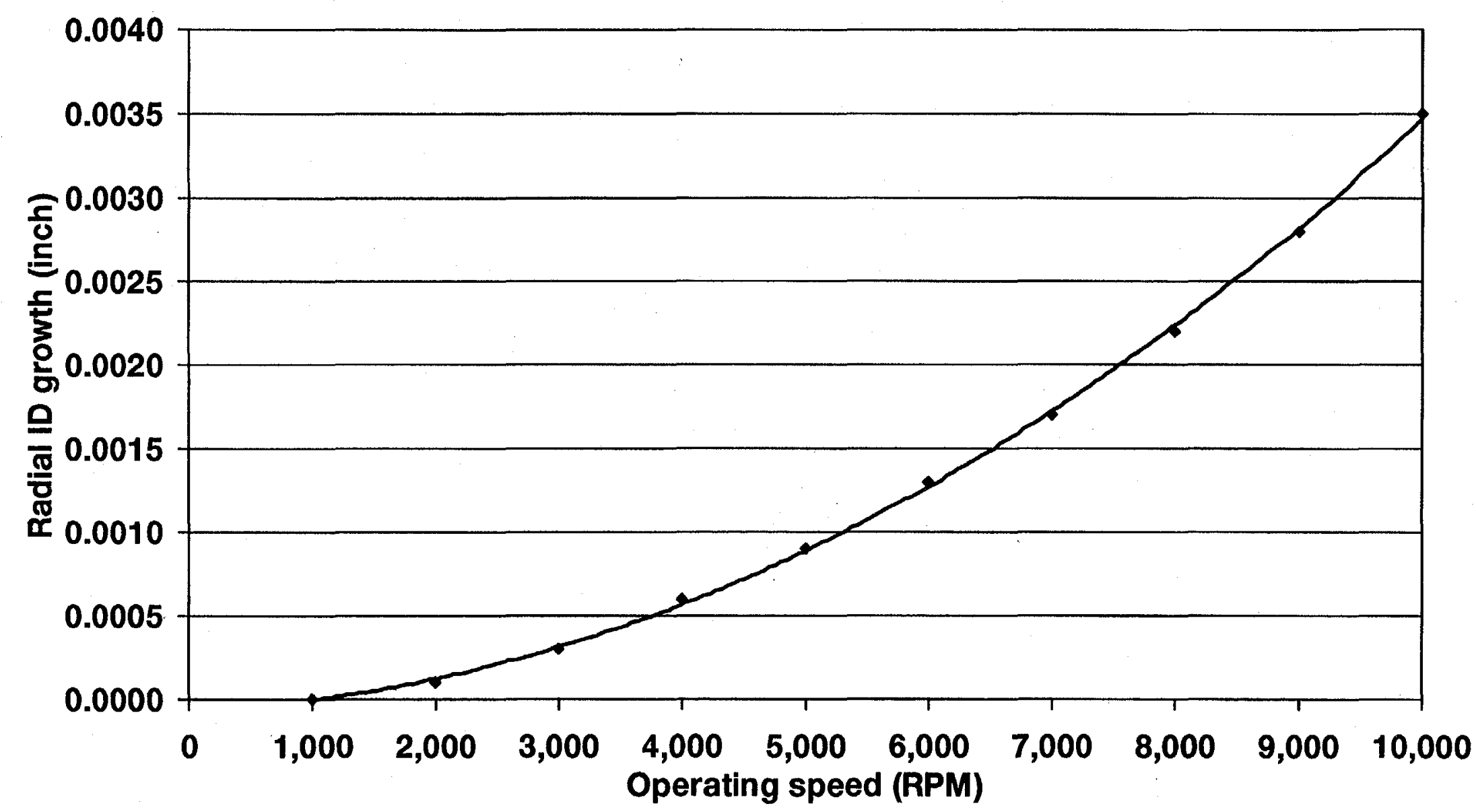

Figure 9. Bearing journal radial expansion versus operating speed. 
39

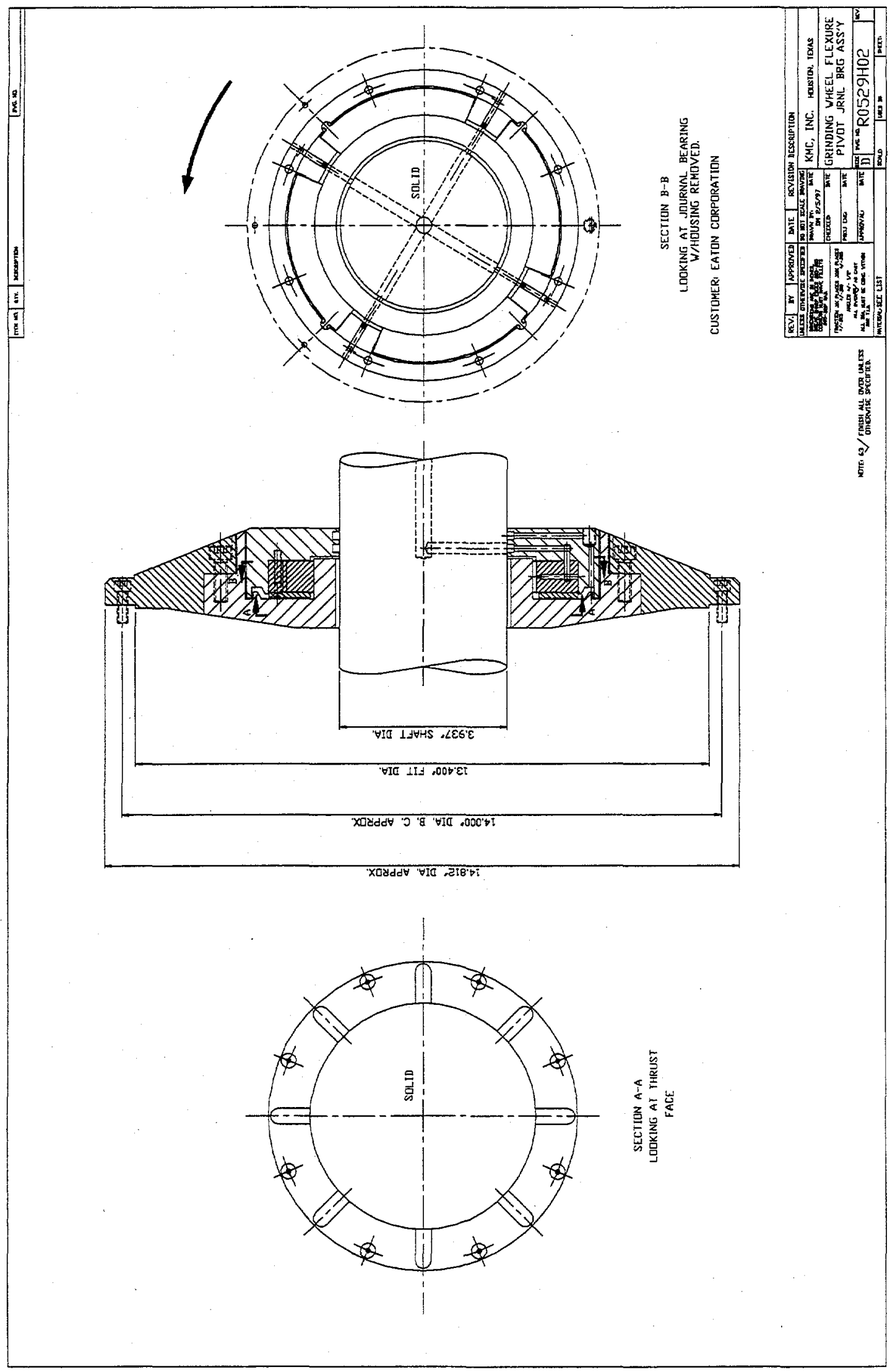

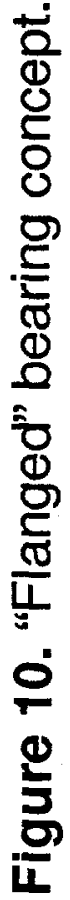




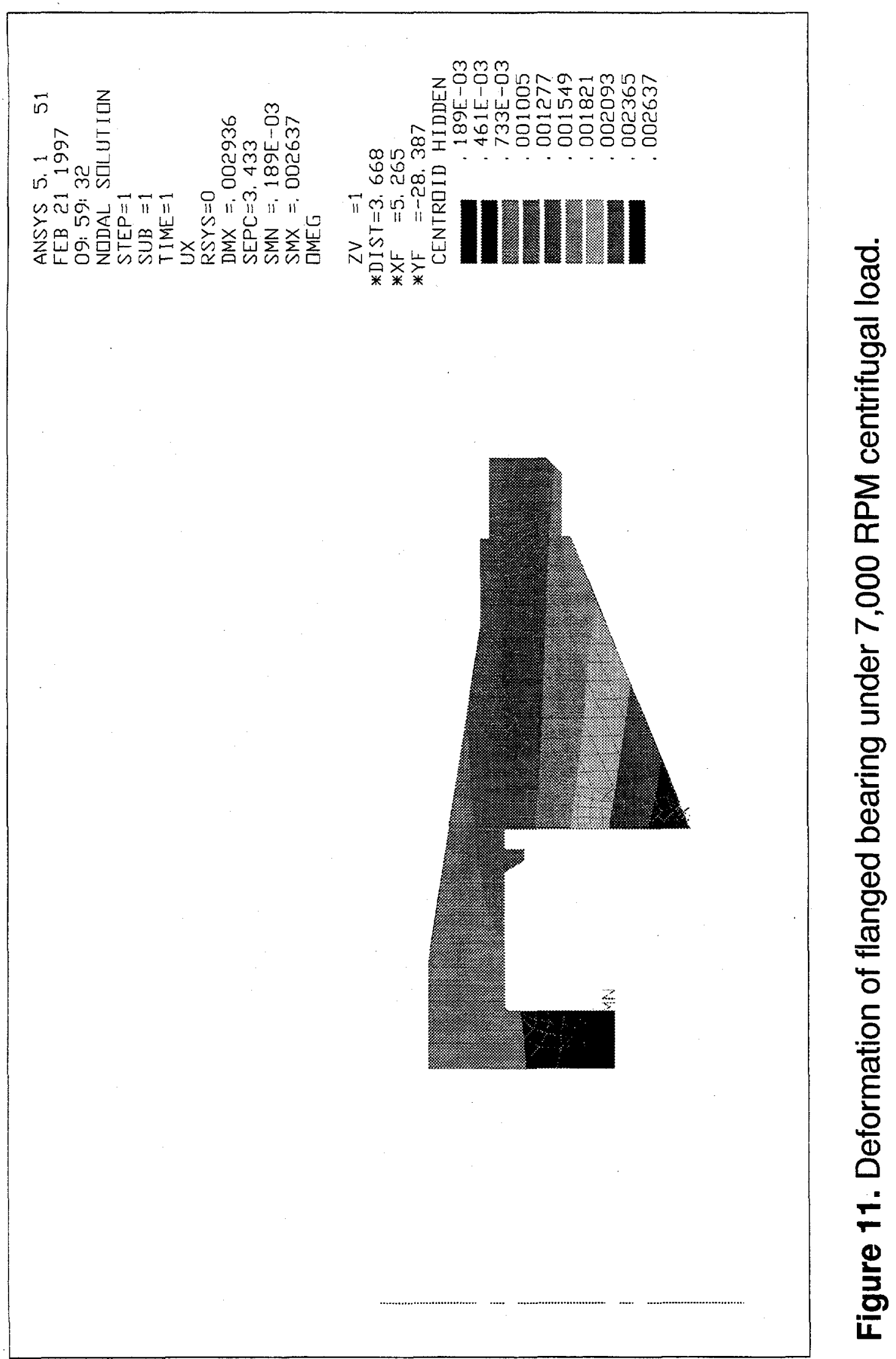




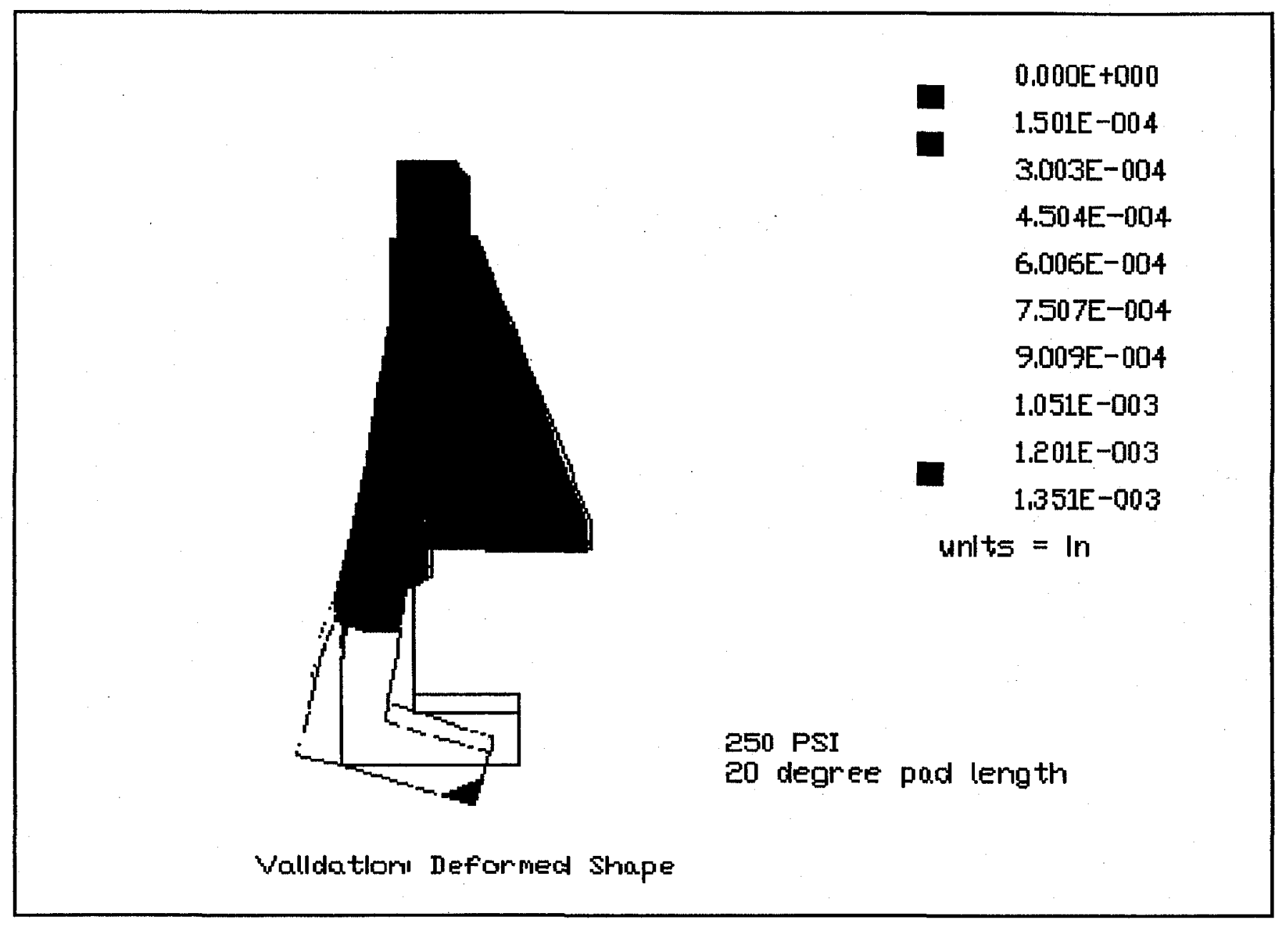

Figure 12. Deformation of flanged bearing under hydrodynamic fluid pressure. 
- Tilting 3 pad (1 actuated) fluid bearing

- Inside-Out (OD Journal) configuration

- Actuated pad used to:

- Compensate for journal expansion at high RPM

- Control overall bearing stiffness

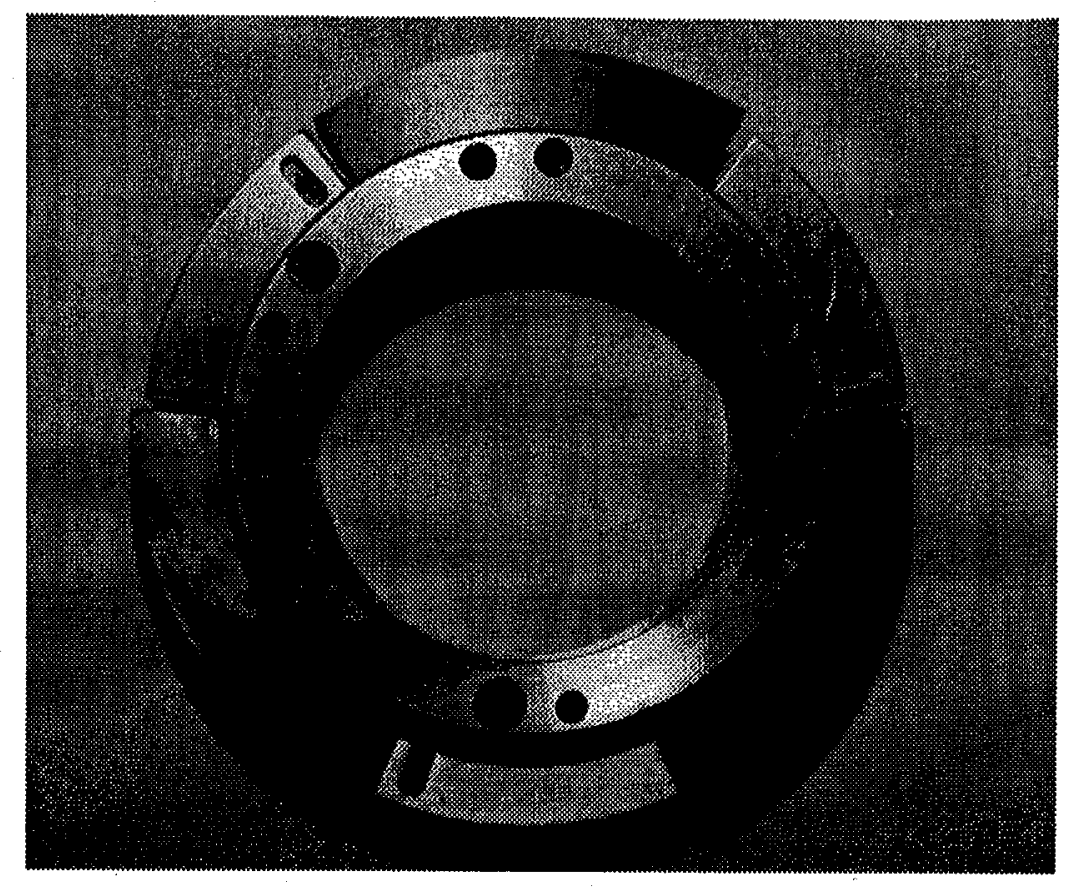

\begin{tabular}{|l|l|}
\hline Operating speed range & $1,250-7,000+\mathrm{RPM}$ \\
\hline Stiffness range & $500,000-1,500,000 \mathrm{lb} / \mathrm{in}$ \\
\hline \multicolumn{1}{|c|}{ Operating Characteristics at $1,000,000 \mathrm{lb} /$ in stiffness and $7,000 \mathrm{RPM}$} \\
\hline Minimum film thickness & 0.0003 inch \\
\hline Power loss & $5.43 \mathrm{HP}$ maximum \\
\hline Peak oil tem perature & $258^{\circ} \mathrm{F}$ \\
\hline Required oil supply & $2.4 \mathrm{GPM} @ 30 \mathrm{PSI}$ \\
\hline
\end{tabular}

Figure 13. Bearing design and operating characteristics. 


\section{Single Pad}

Predicted Load Capacity vs. Misalignment

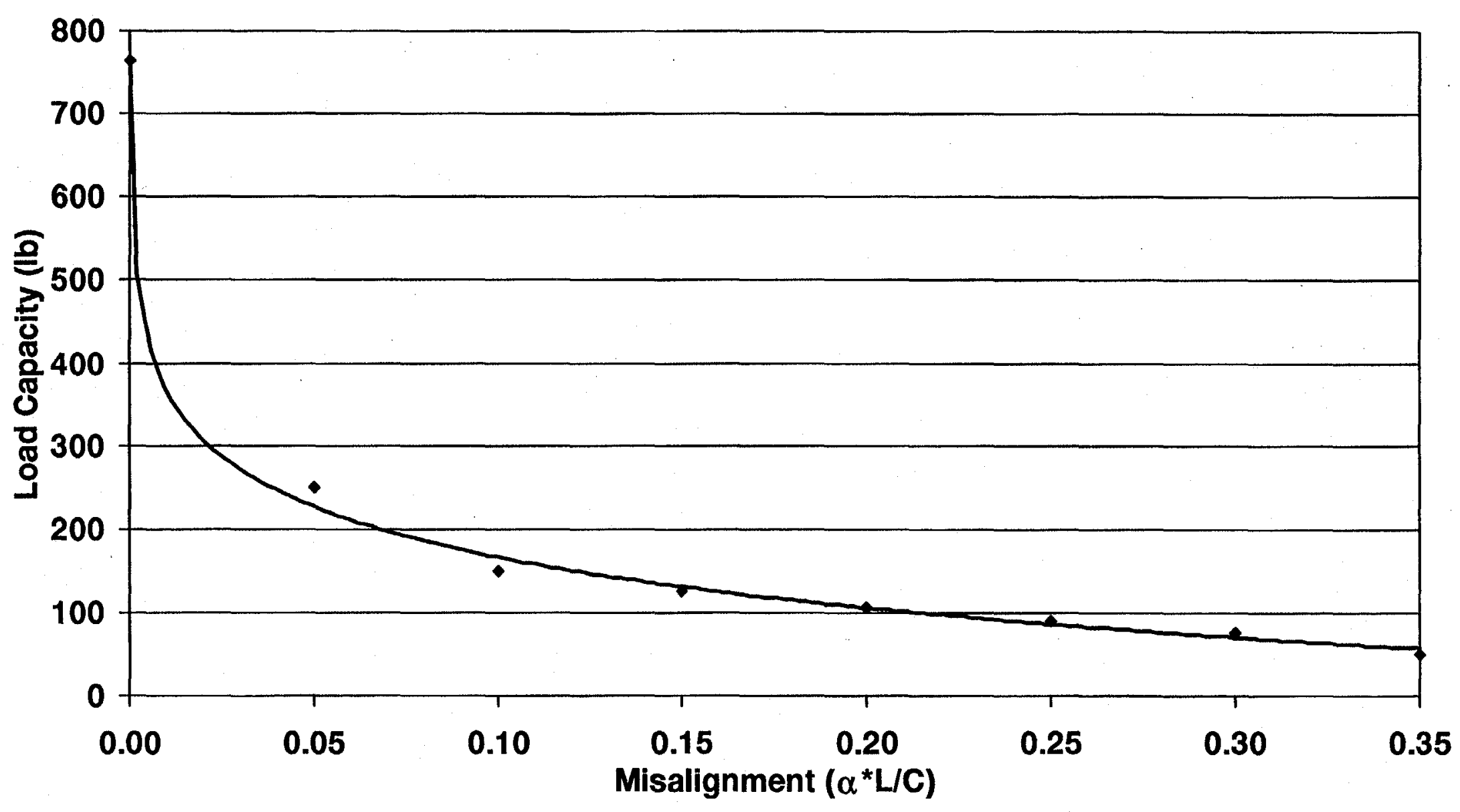

Figure 14. Effect of shaft misalignment on bearing load capacity. 
Actuated 3-Pad Bearing

Required Preload versus Speed

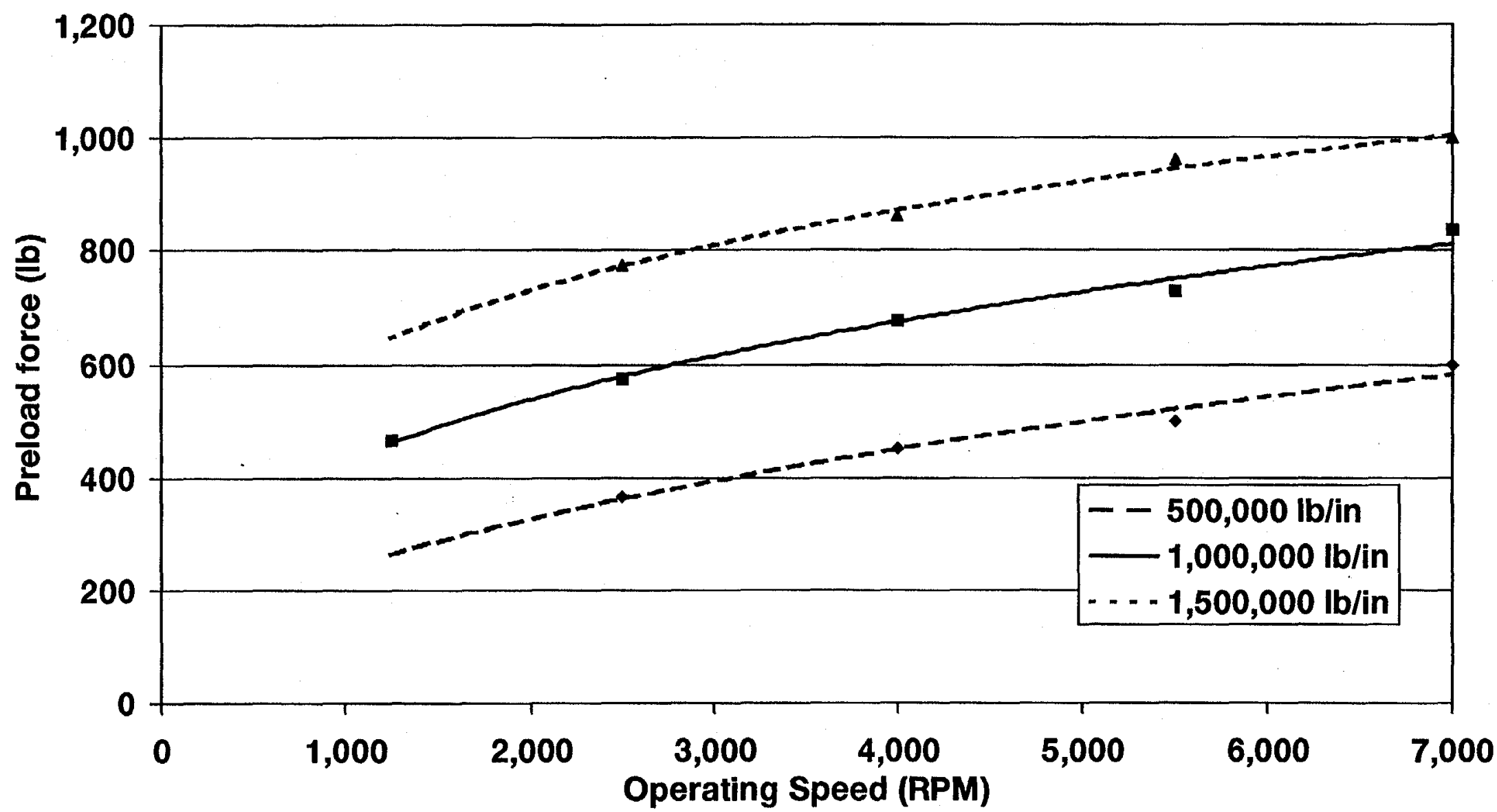

Figure 15. Required preload to maintain constant stiffness over operating speed range. 


\section{Actuated 3-Pad Bearing}

Predicited Minimum Film Thickness versus Speed

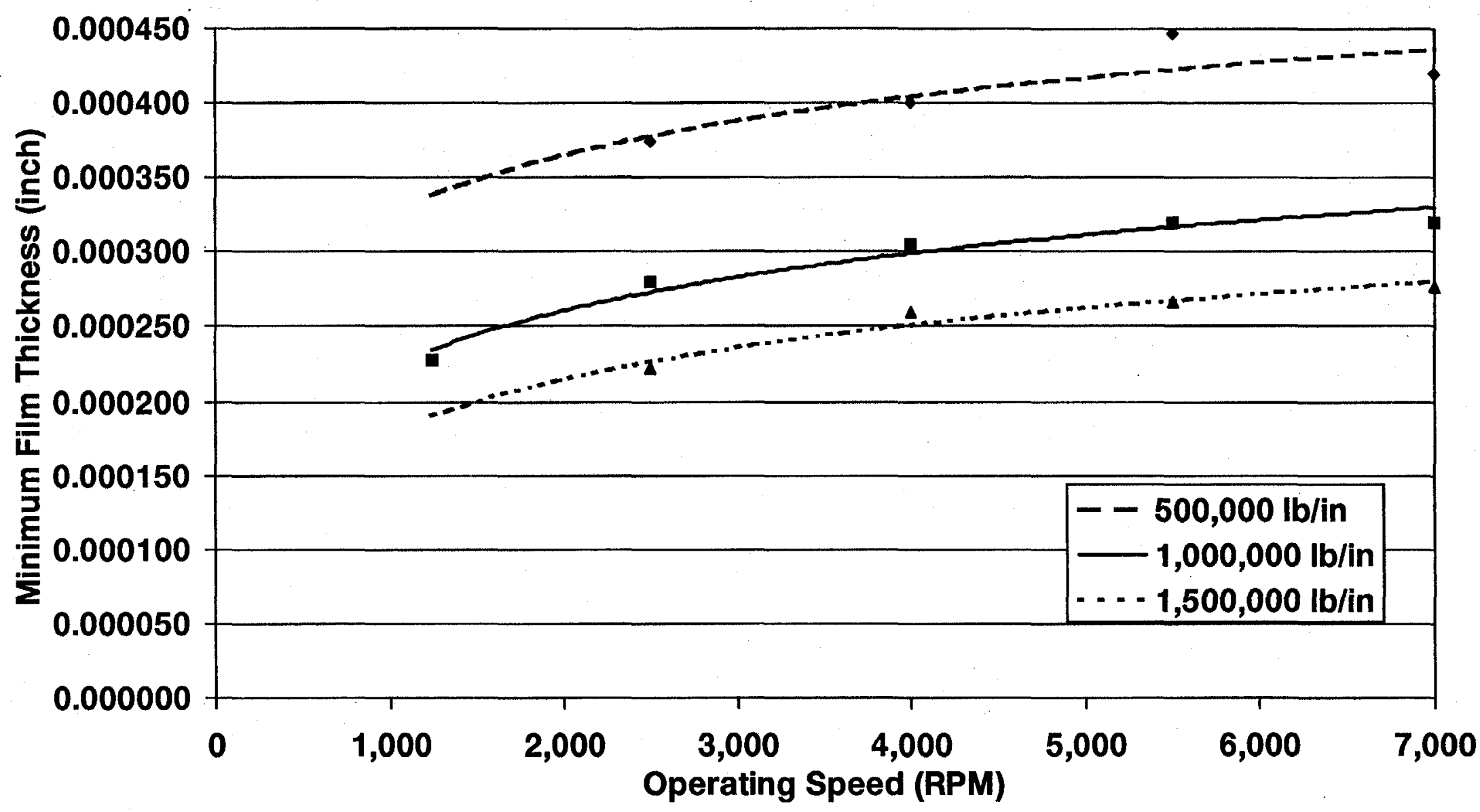

Figure 16. Minimum film thickness versus operating speed. 
Actutated 3-Pad Bearing

Predicted Power Loss versus Speed

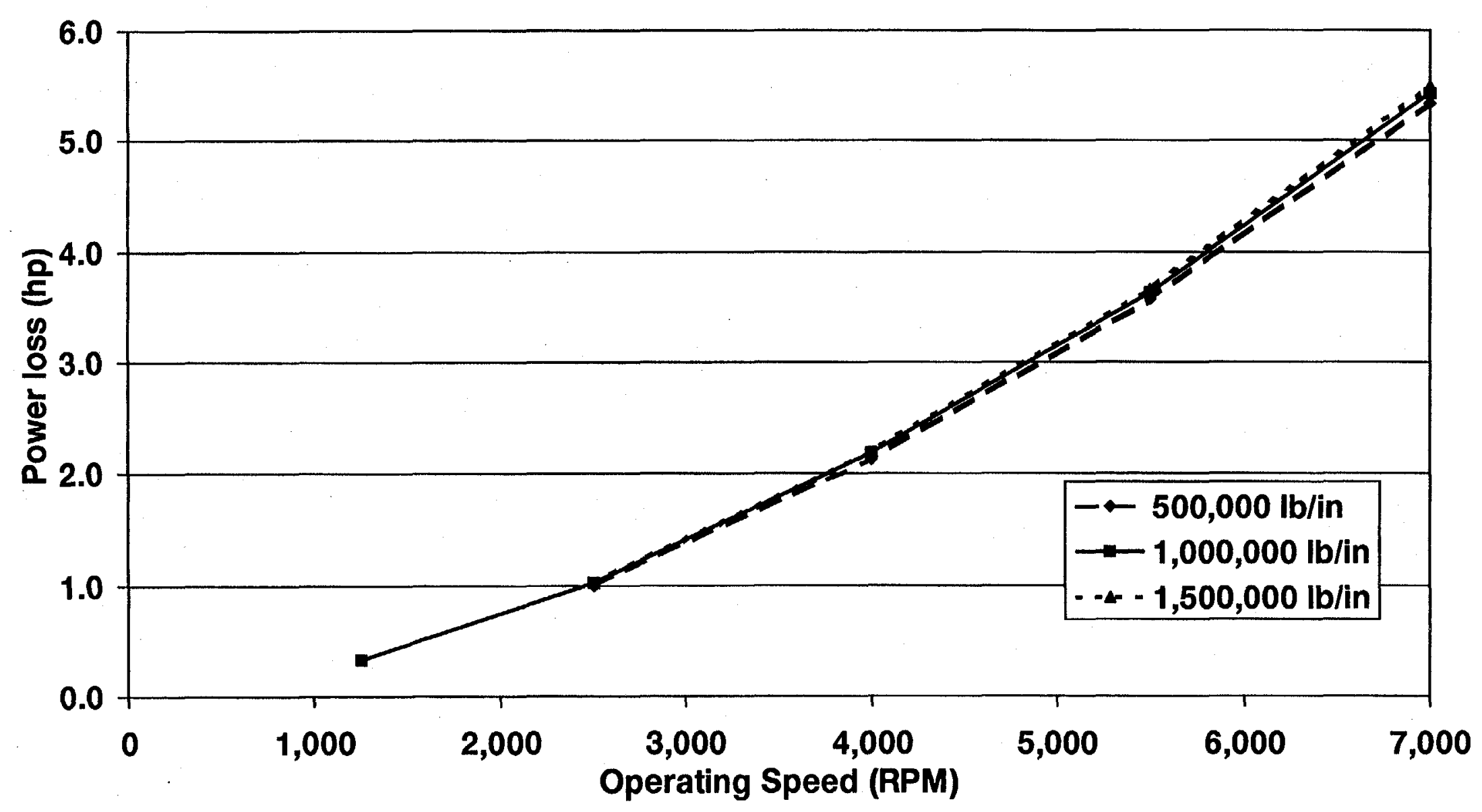

क)

Figure 17. Power lost in bearing versus operating speed. 
Actuated 3-Pad Bearing

Predicted Flow versus Speed

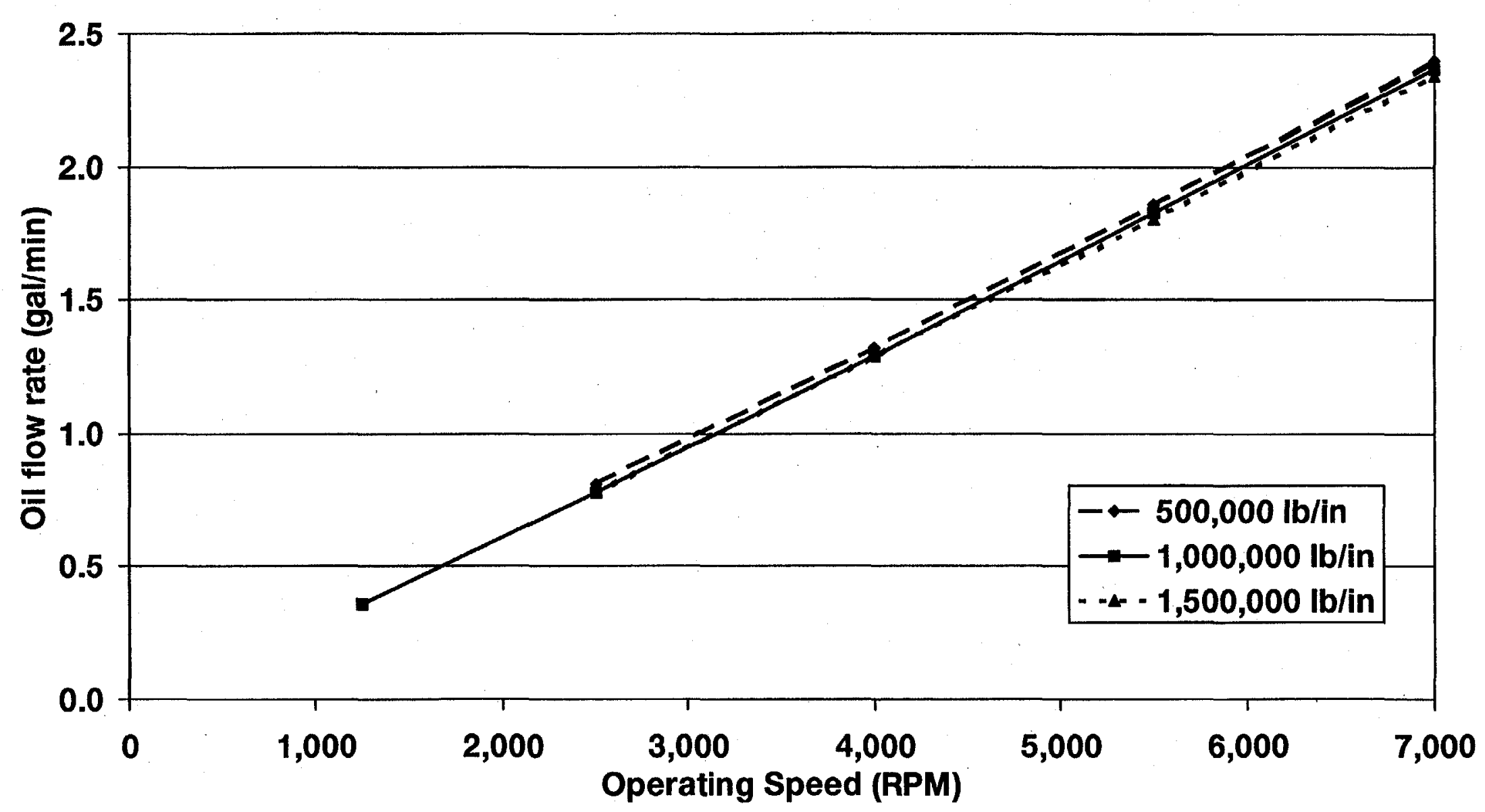

Figure 18. Oil flow rate as a function of operating speed. 


\section{Actuated 3-Pad Bearing}

\section{Predicted Peak Film Temperature versus Speed}

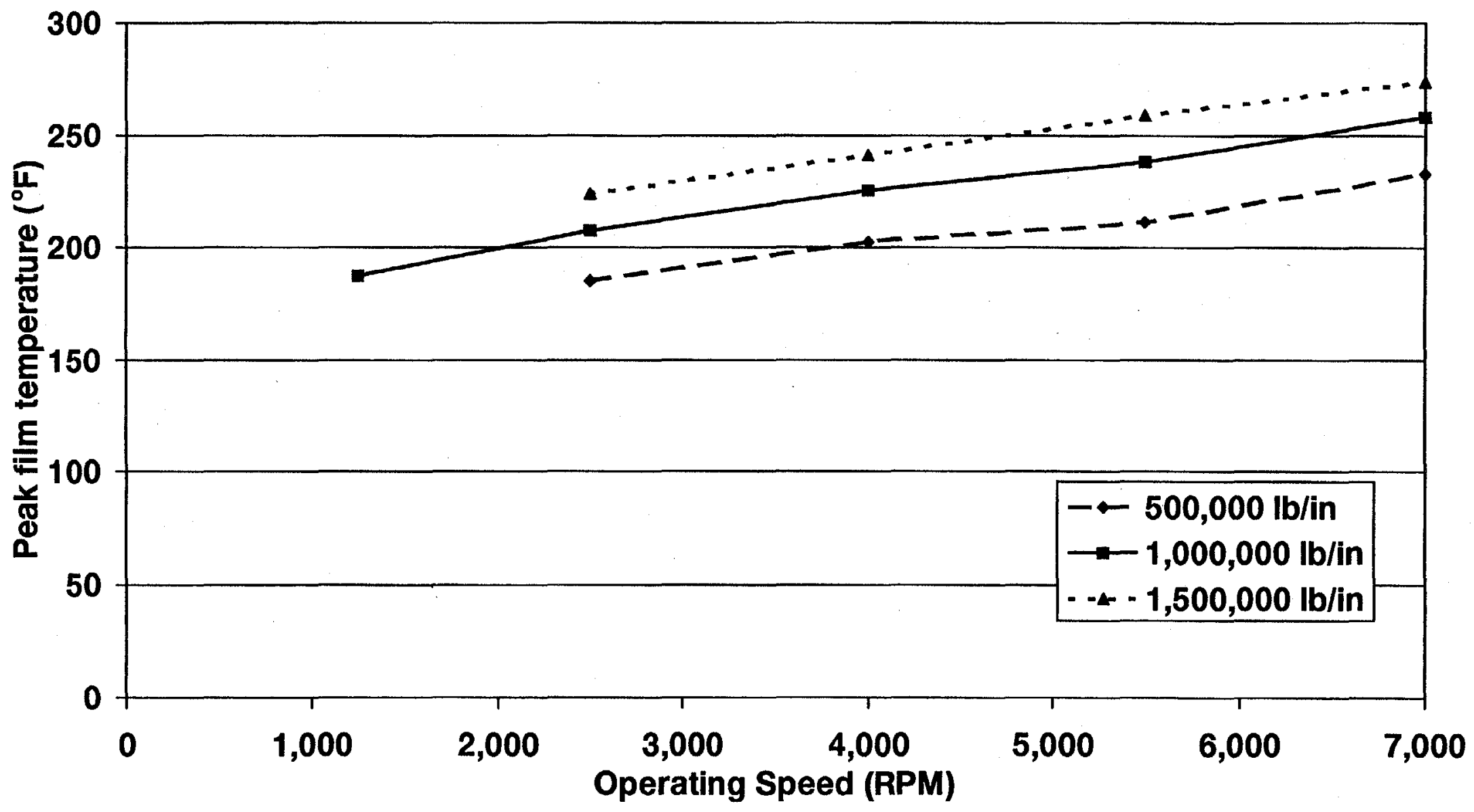

Figure 19. Peak film temperature as function of operating speed. 


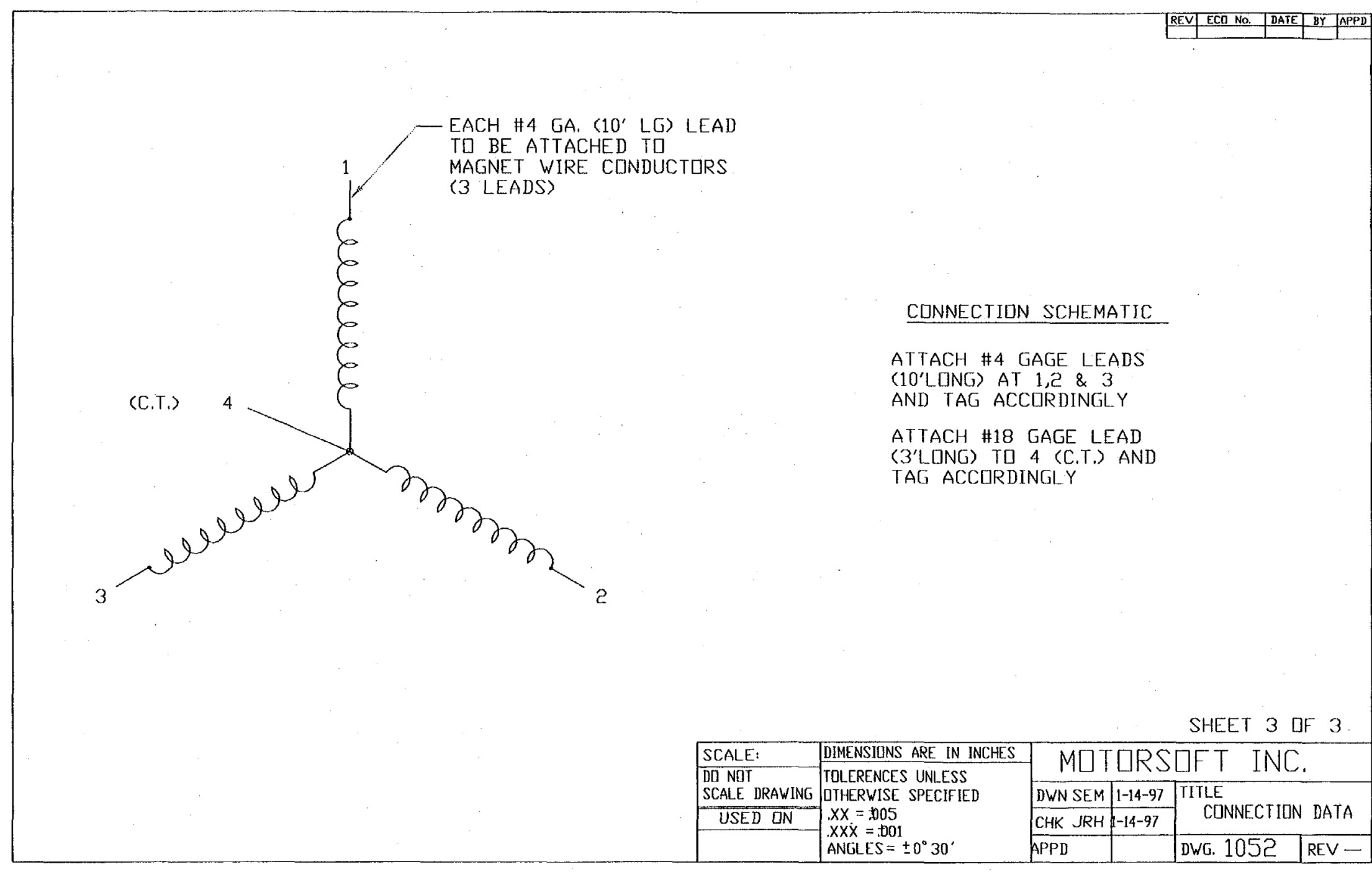

Figure 20. Connection schematic. 


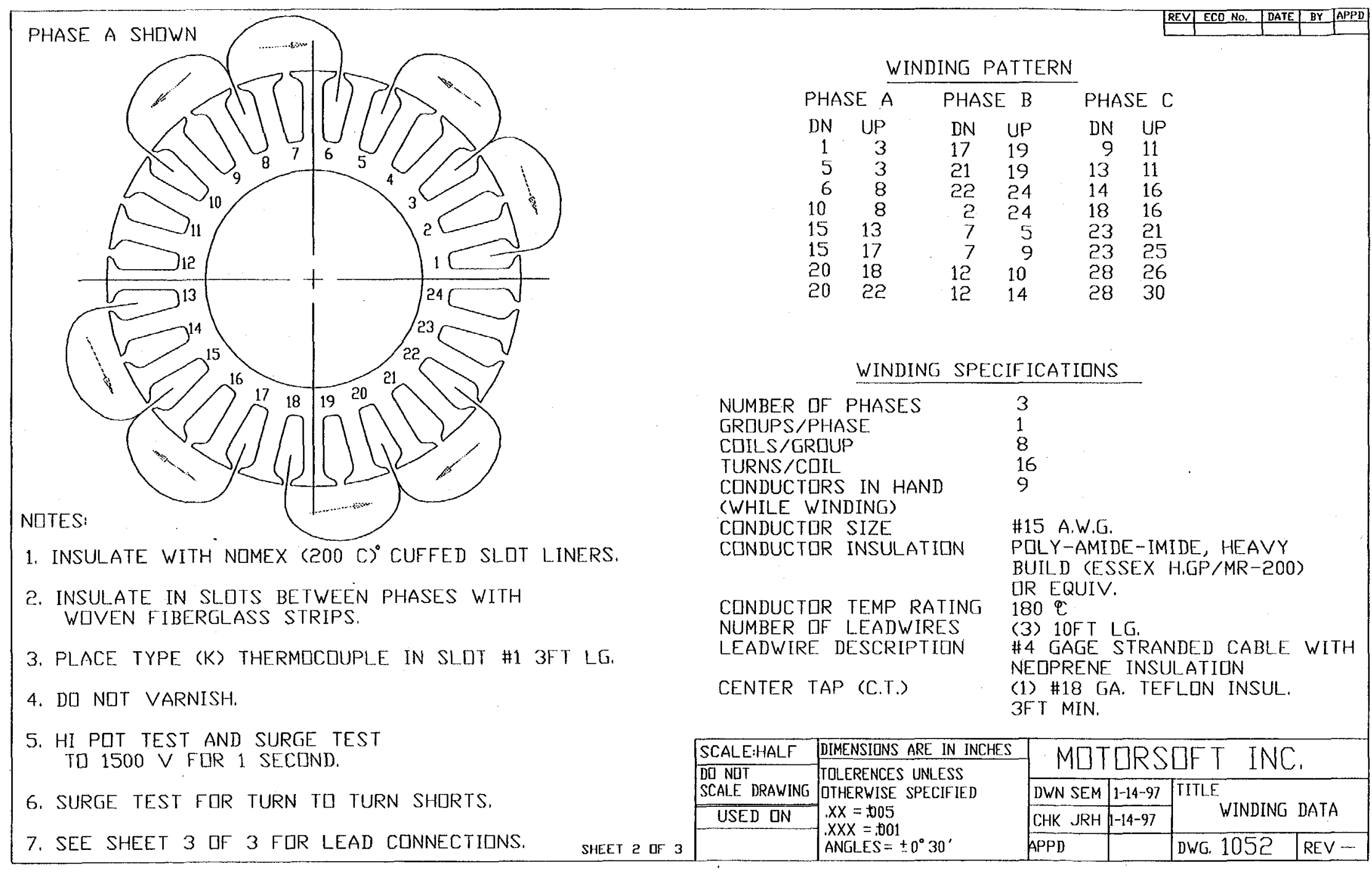

Figure 21. Winding specifications. 

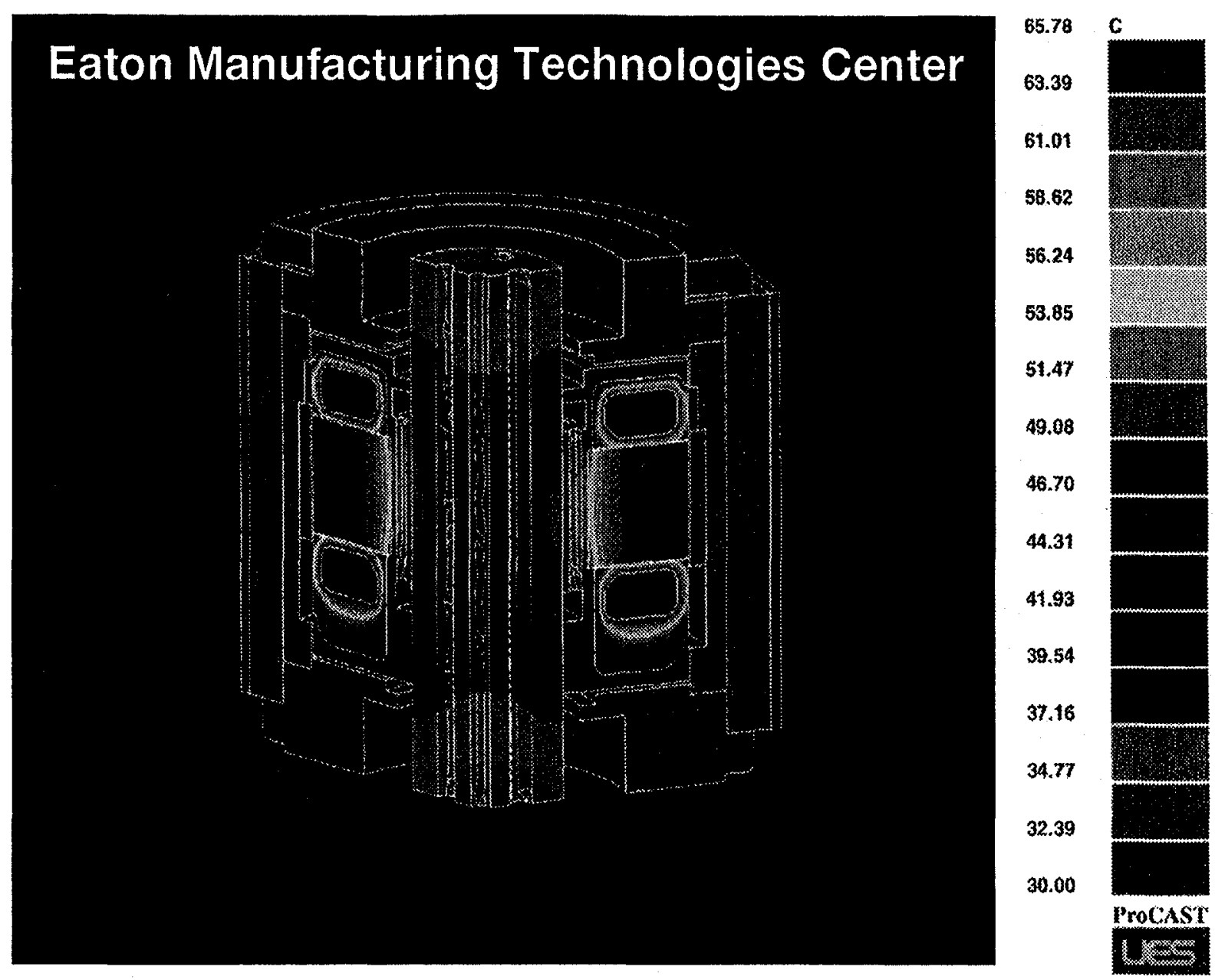

- $1.9 \mathrm{HP}$ loss at 7,000 RPM under full load (63 HP)

$>150^{\circ} \mathrm{F}$ Steady state temperature reached after 40 minutes using internal water cooling

Figure 22. Predicted temperature distribution of spindle under steady-state full power operation. 


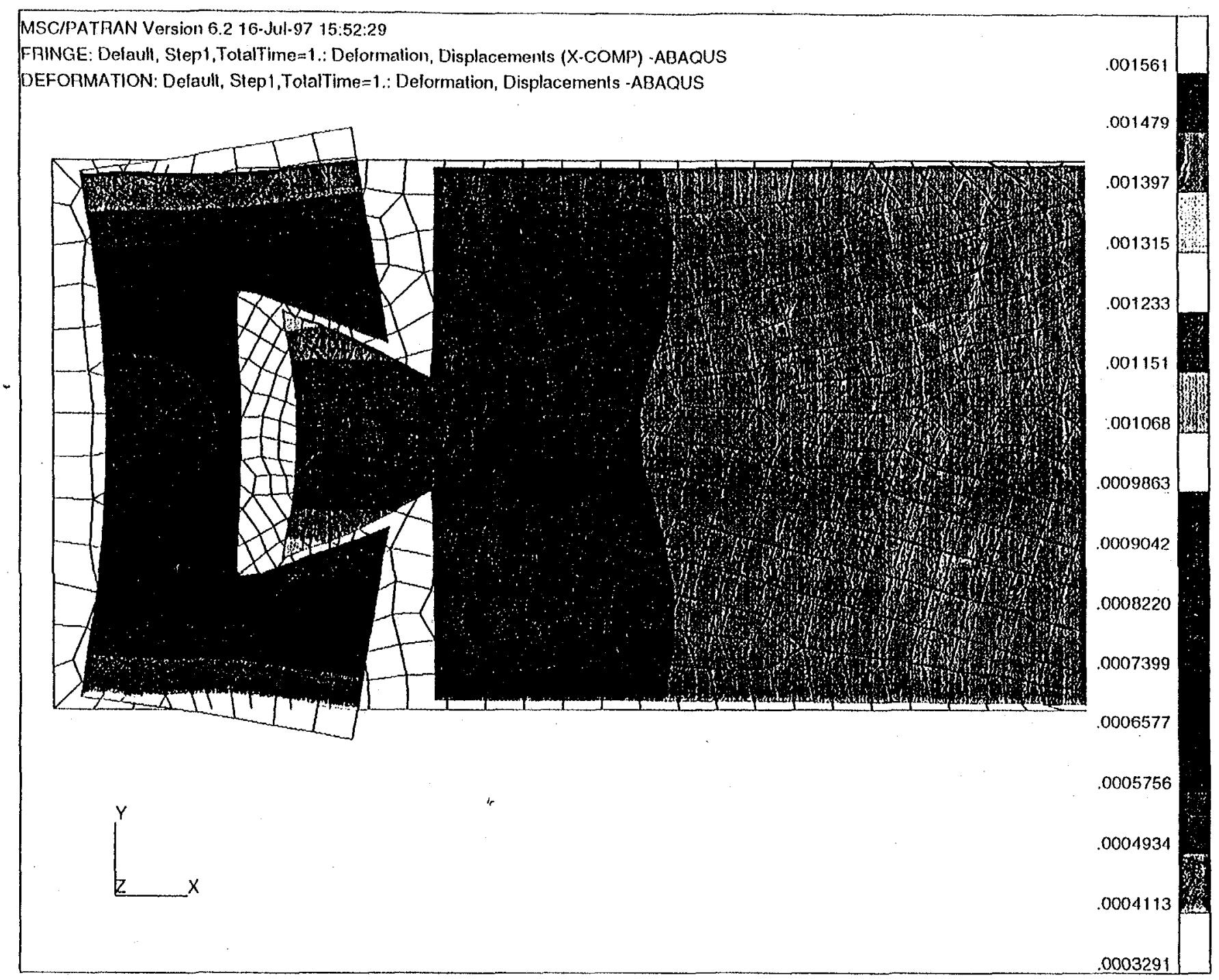

Figure 23. Separation of two-piece side plate under 7,000 RPM operation. 
53

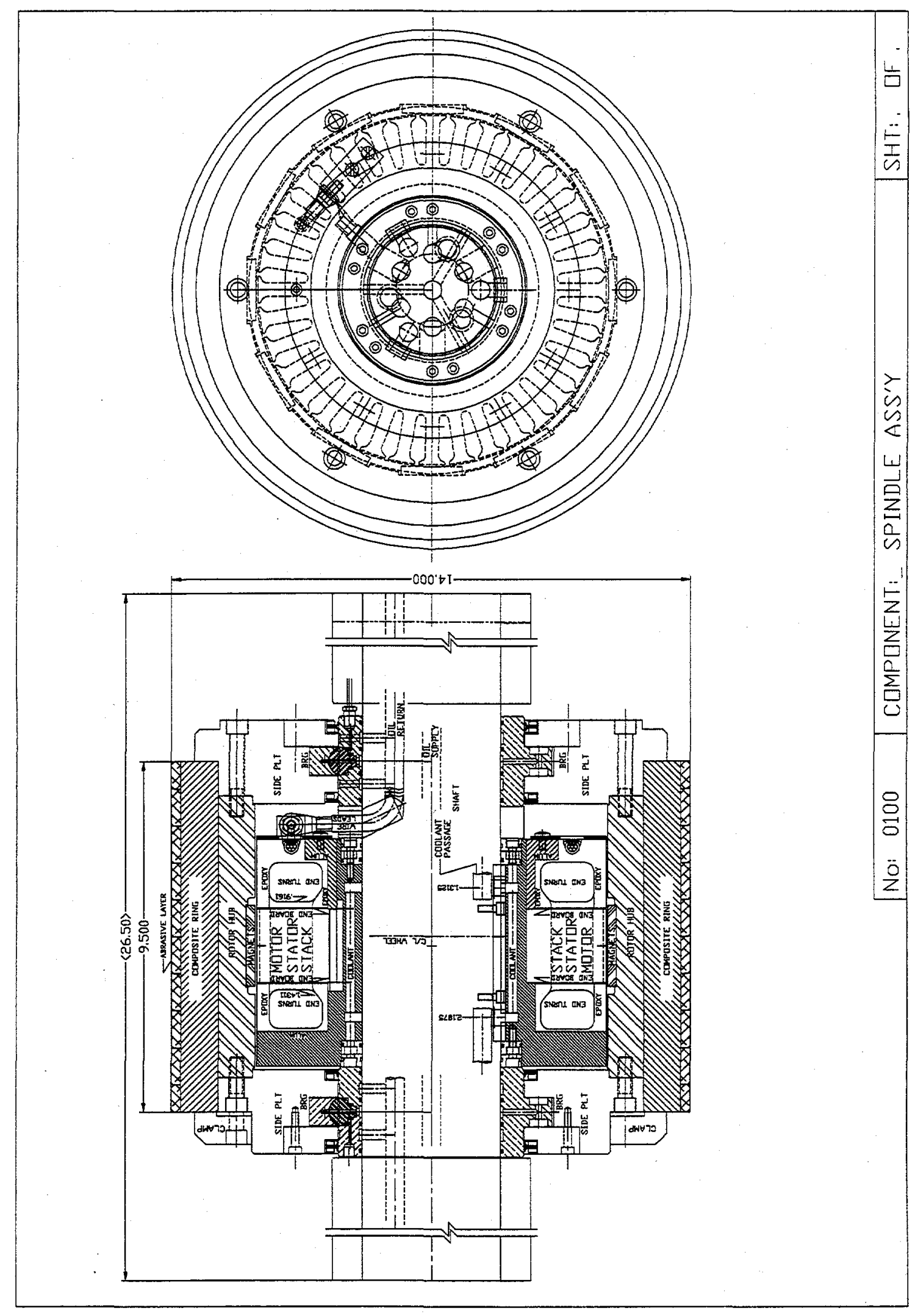




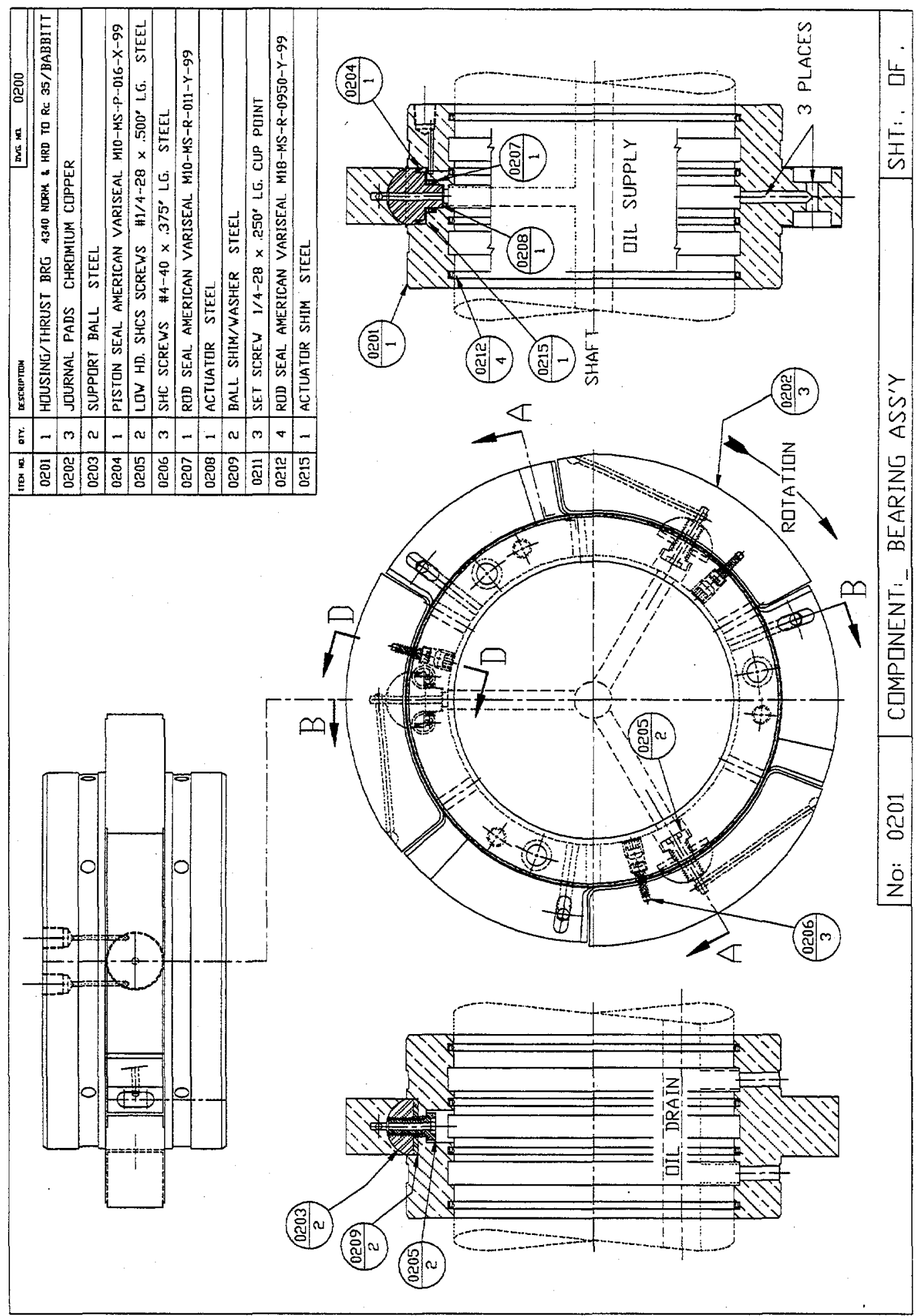


55

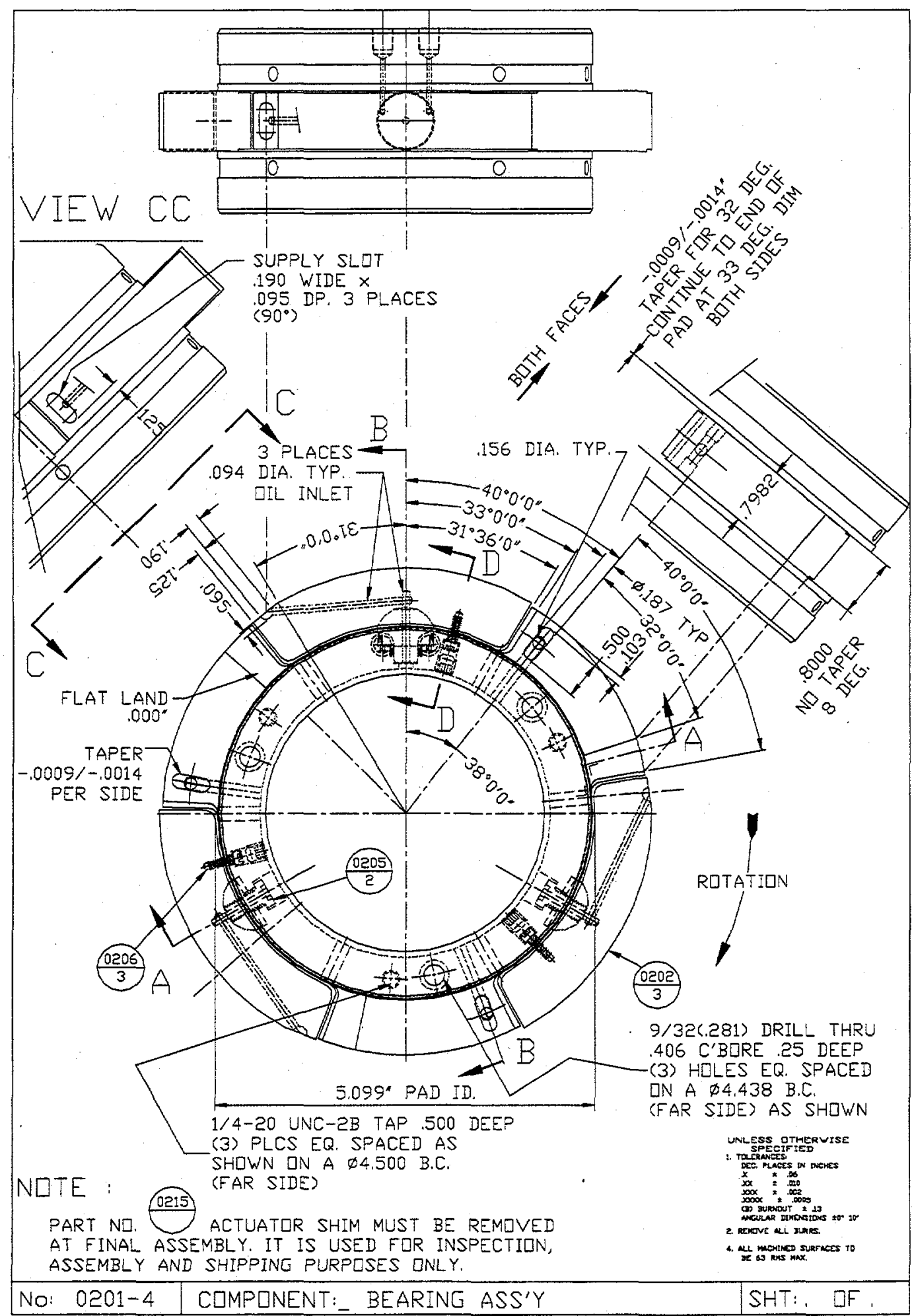

Drawing 3. Bearing design details - thrust and radial pads. 
56

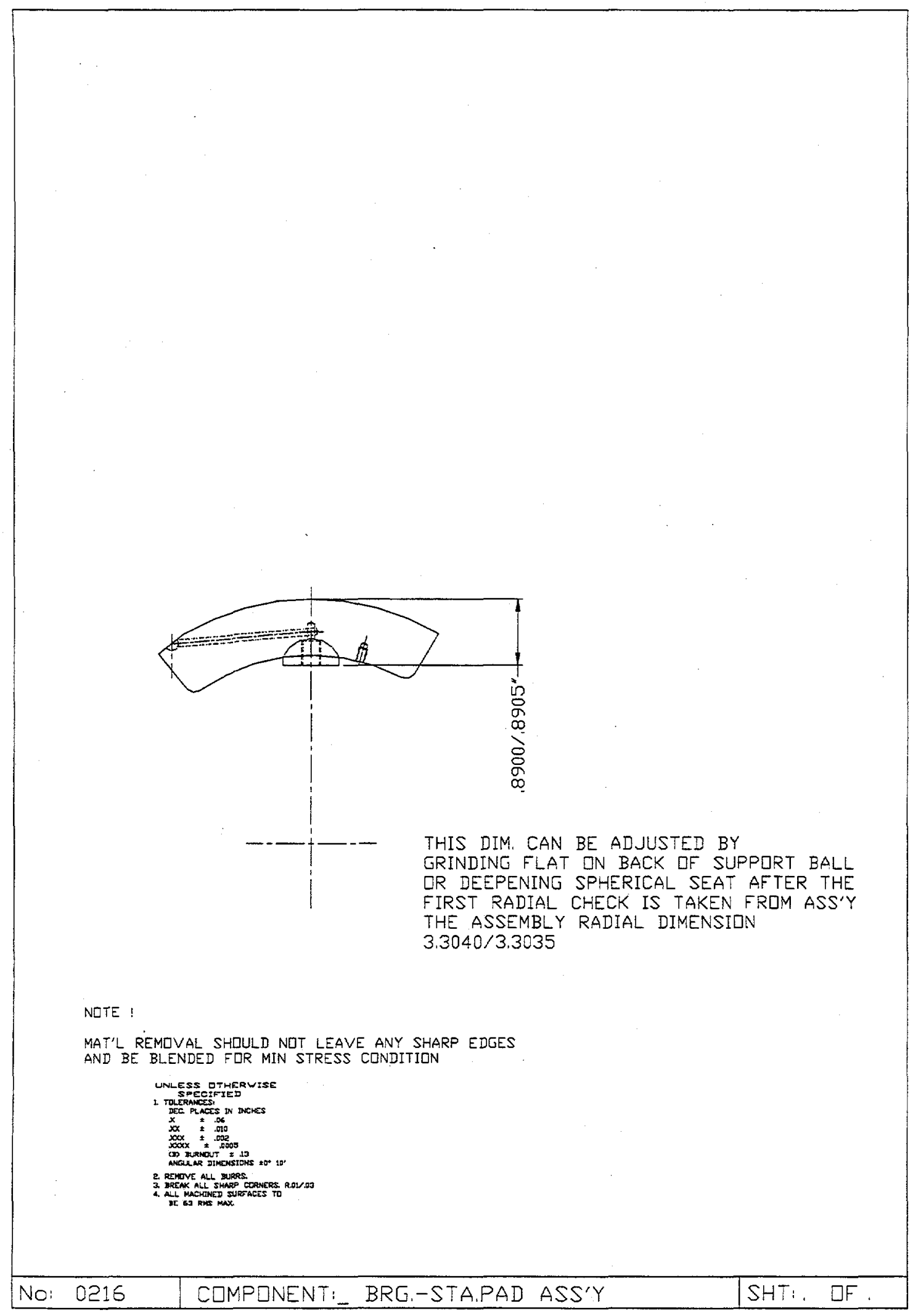

Drawing 4. Tilting pad assembly (tilt on fixed location pivot). 


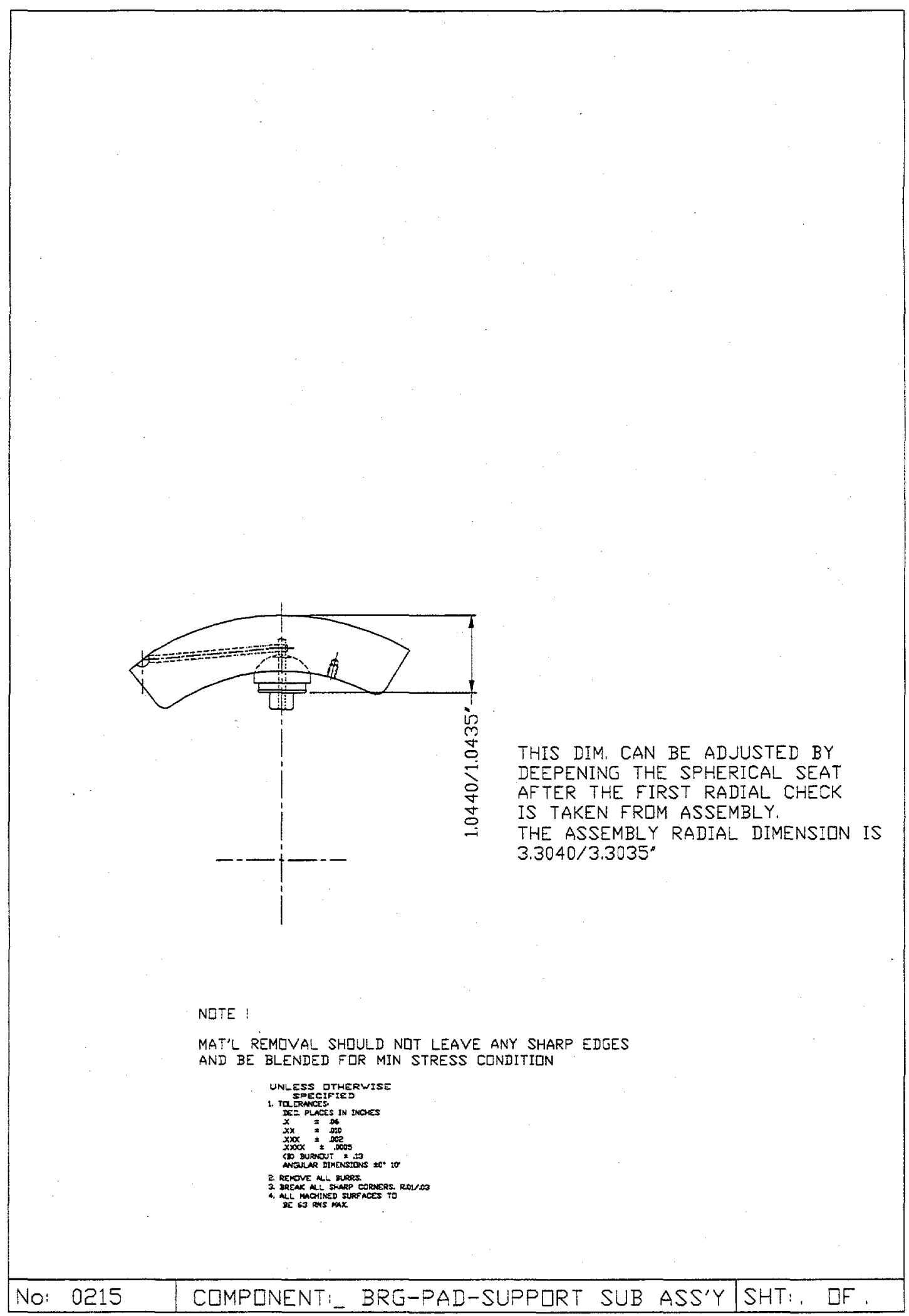

Drawing 5. Tilting pad assembly (tilt on actuator). 


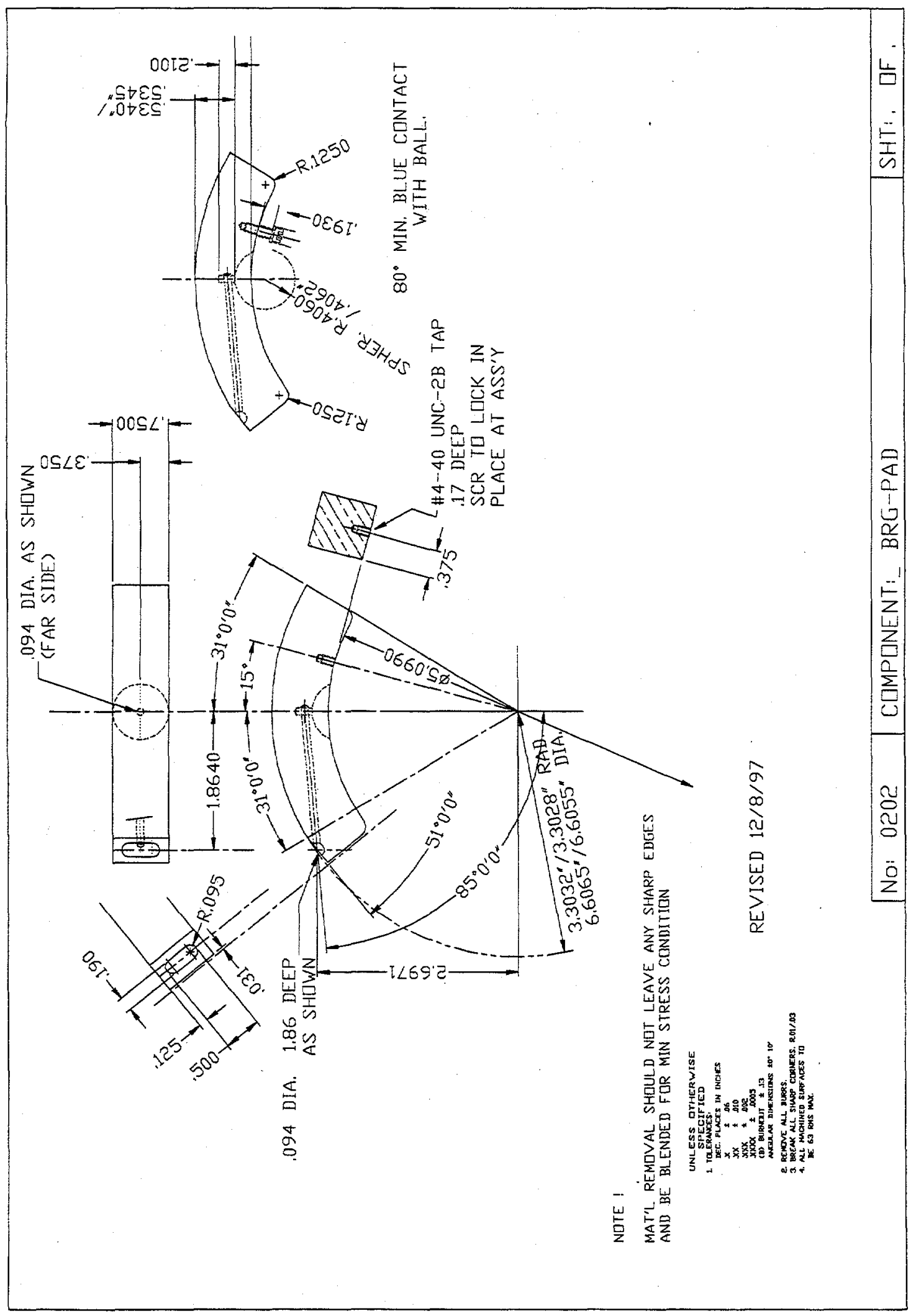


59

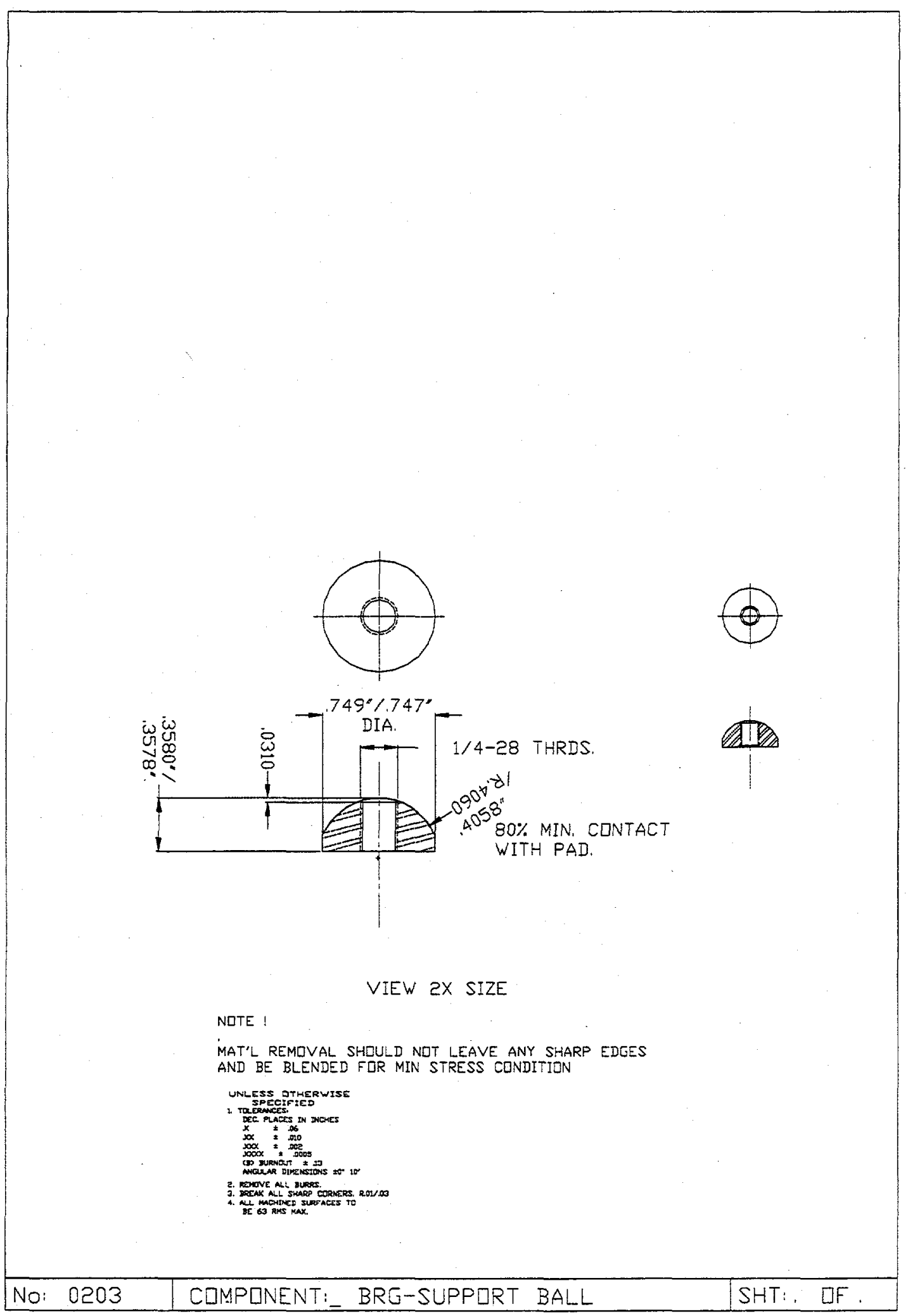

\section{Drawing 7. Fixed location pivot detail.}




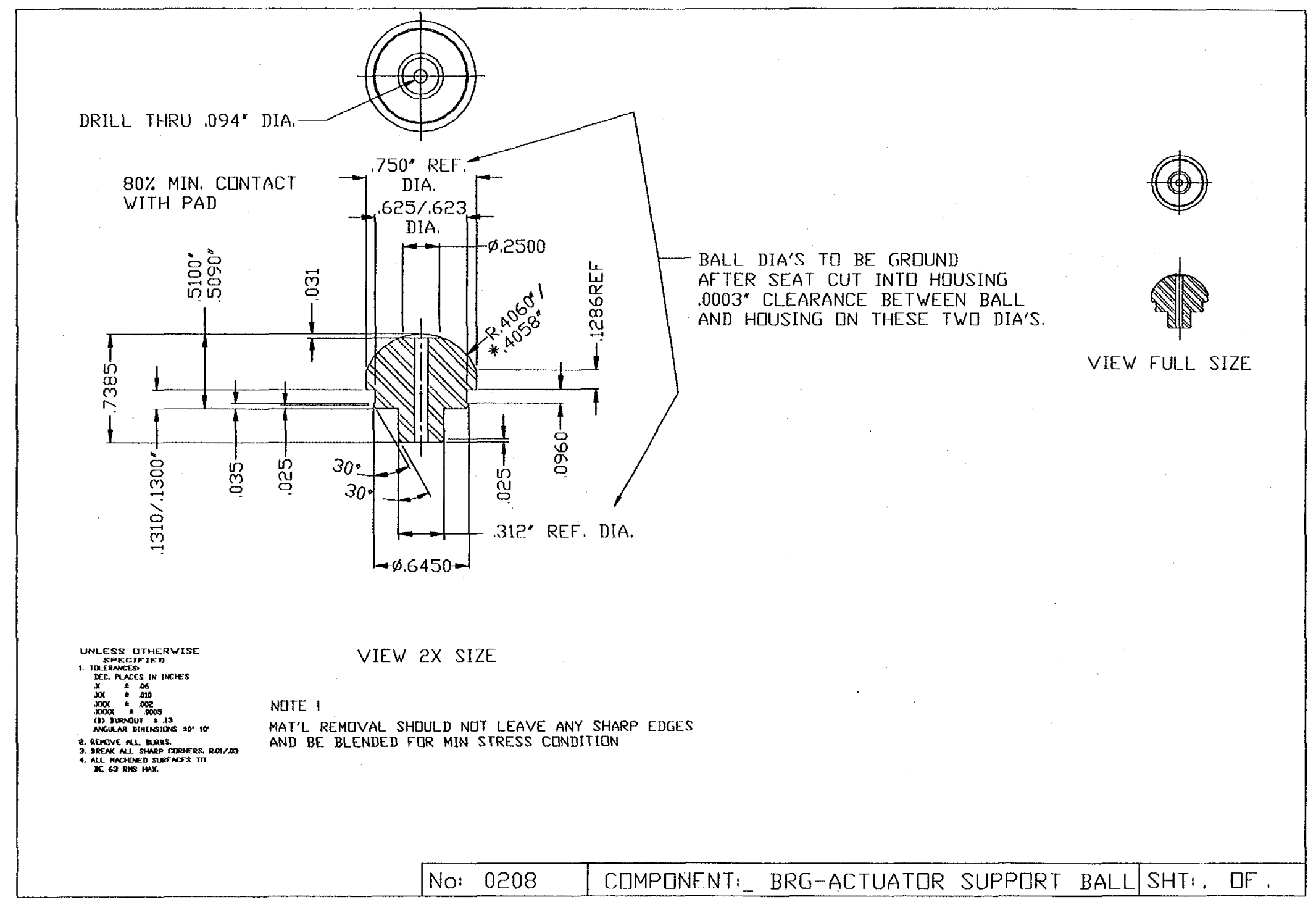




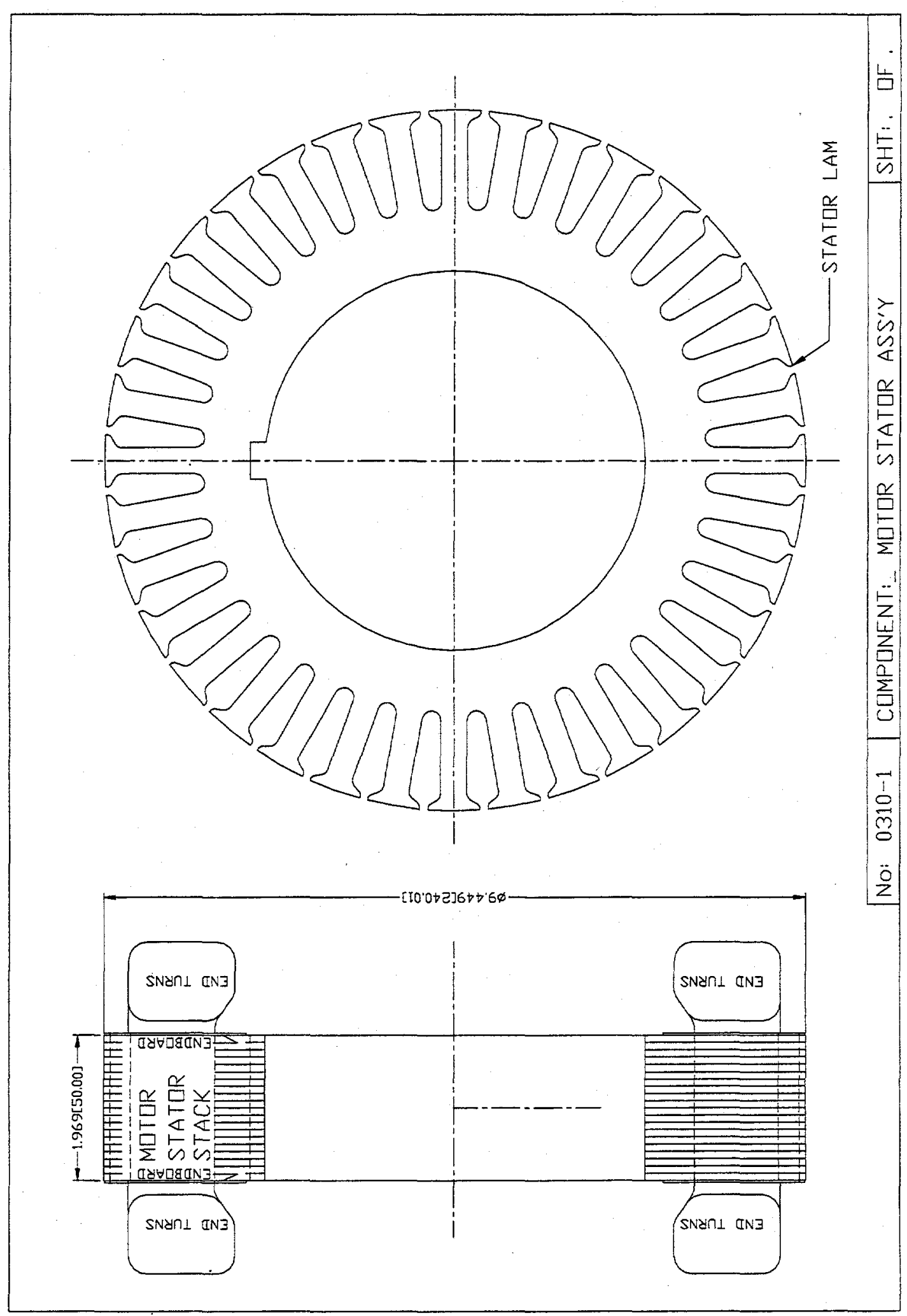

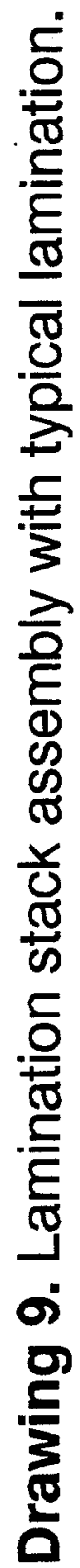


62

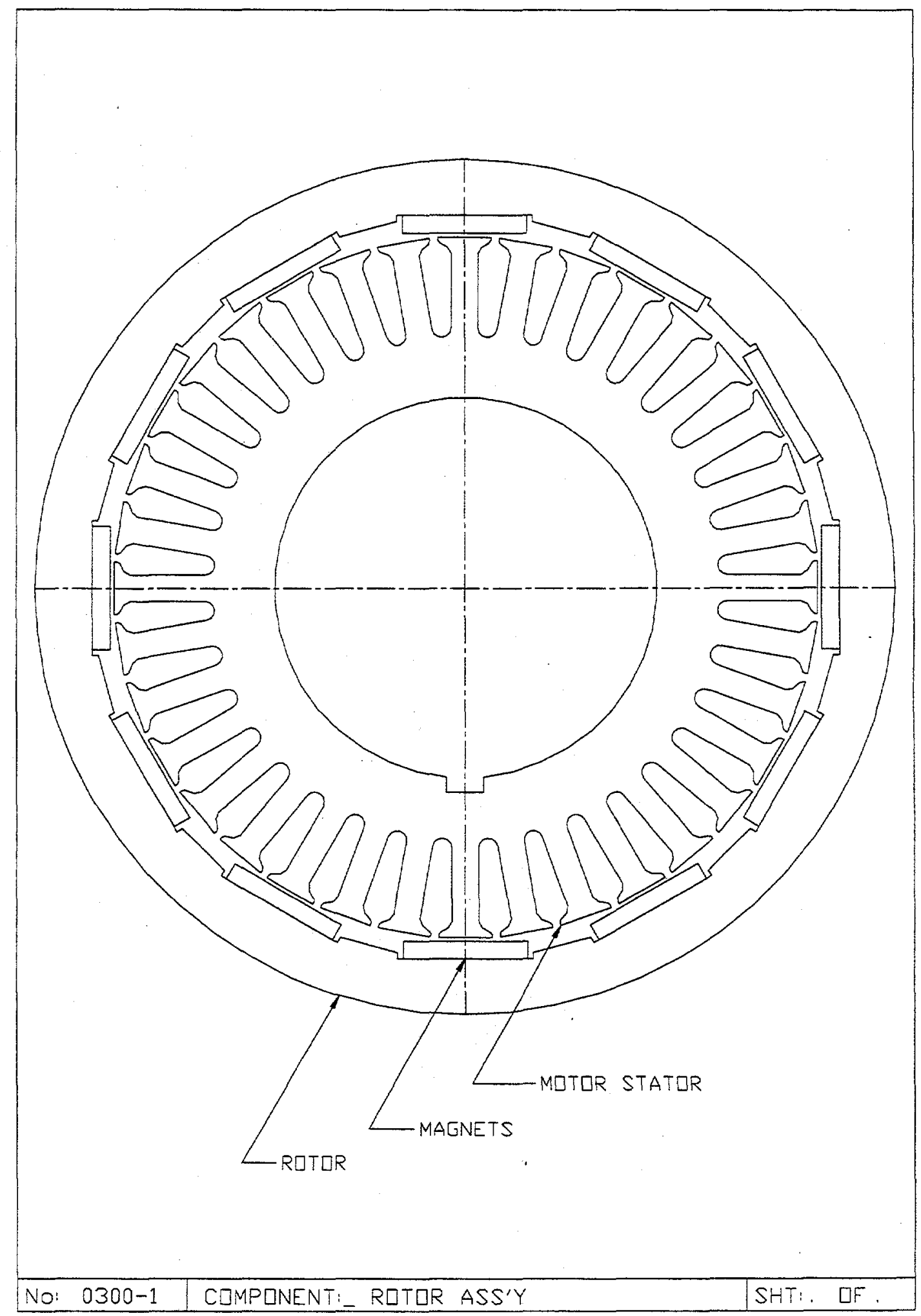

Drawing 10. Motor assembly schematic (stator and rotor end view). 

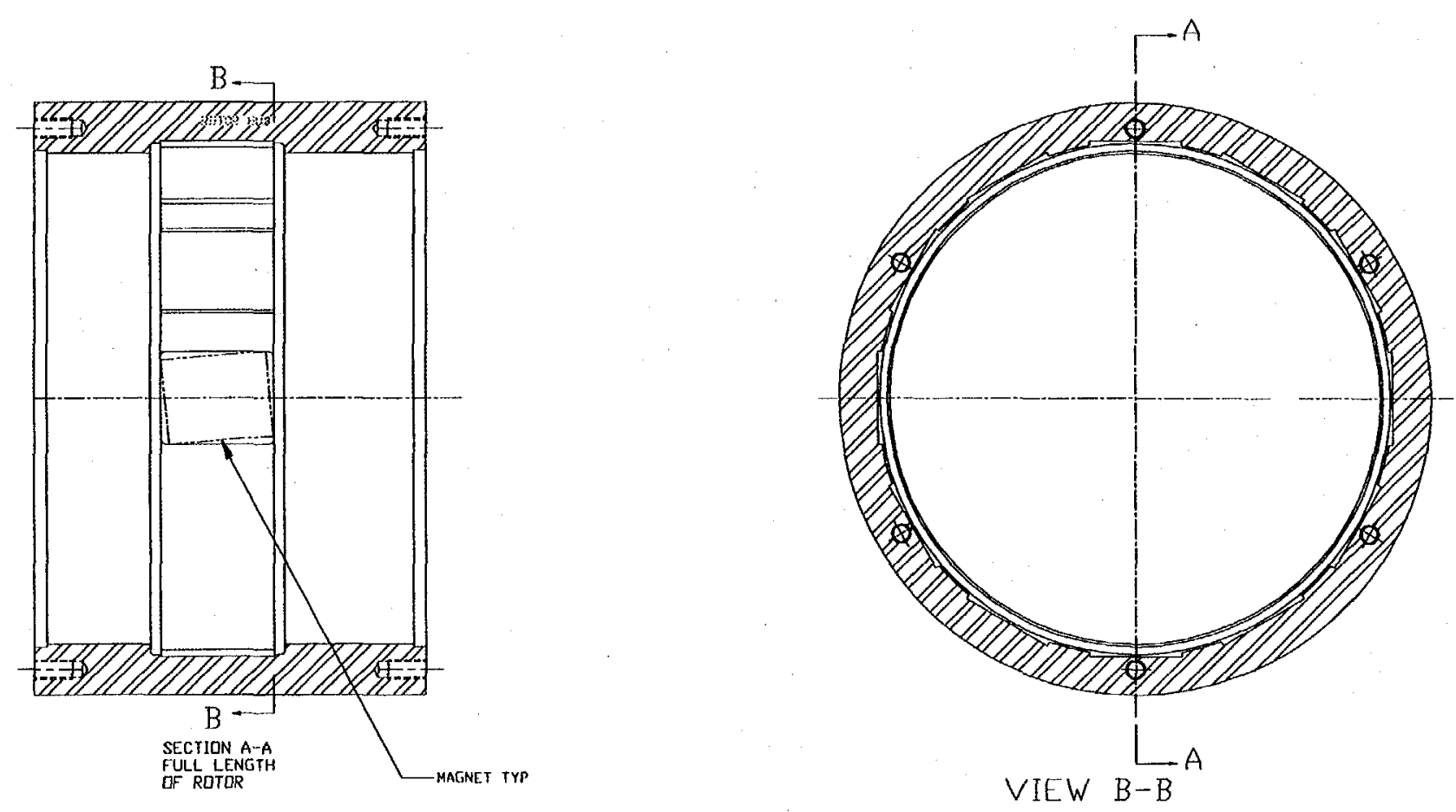

Ф

Drawing 11. Motor hub with skewed magnet.

(0315) 
64

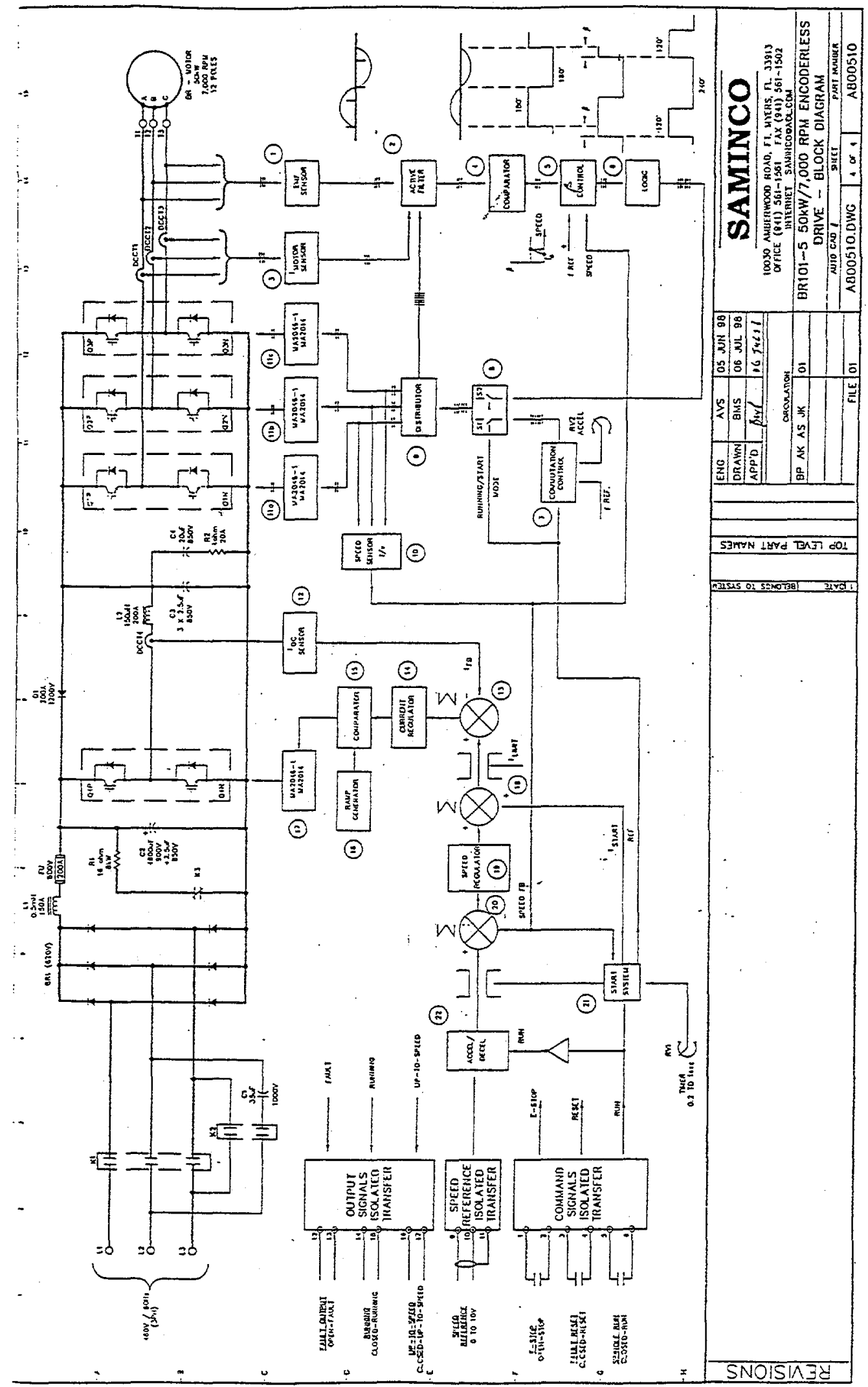

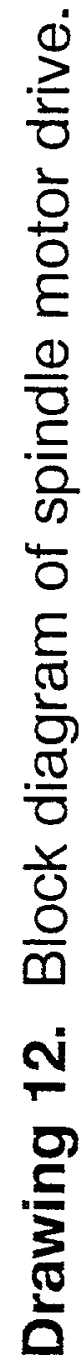




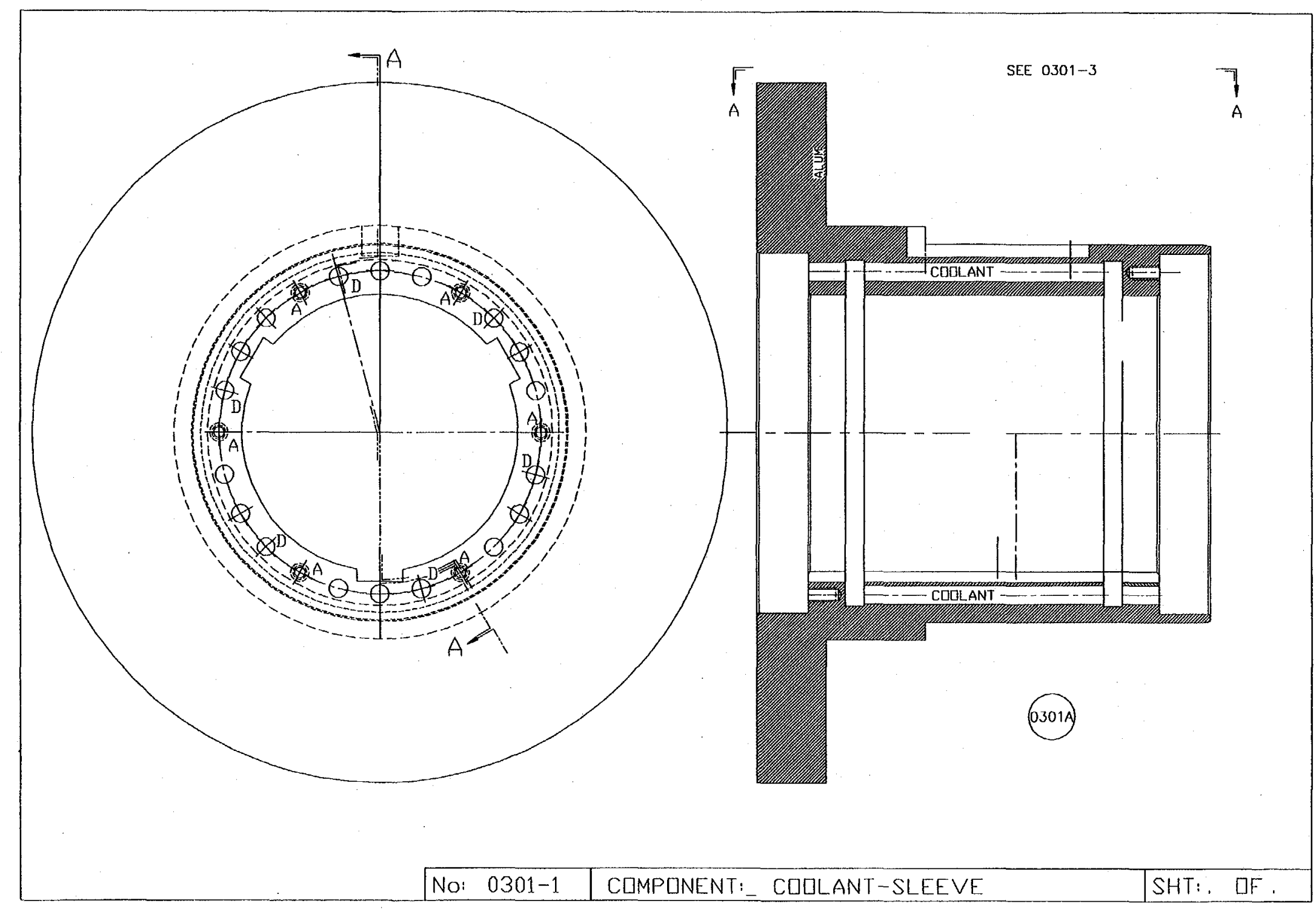

G

Drawing 13. Motor cooling sleeve - cooling passages. 
66

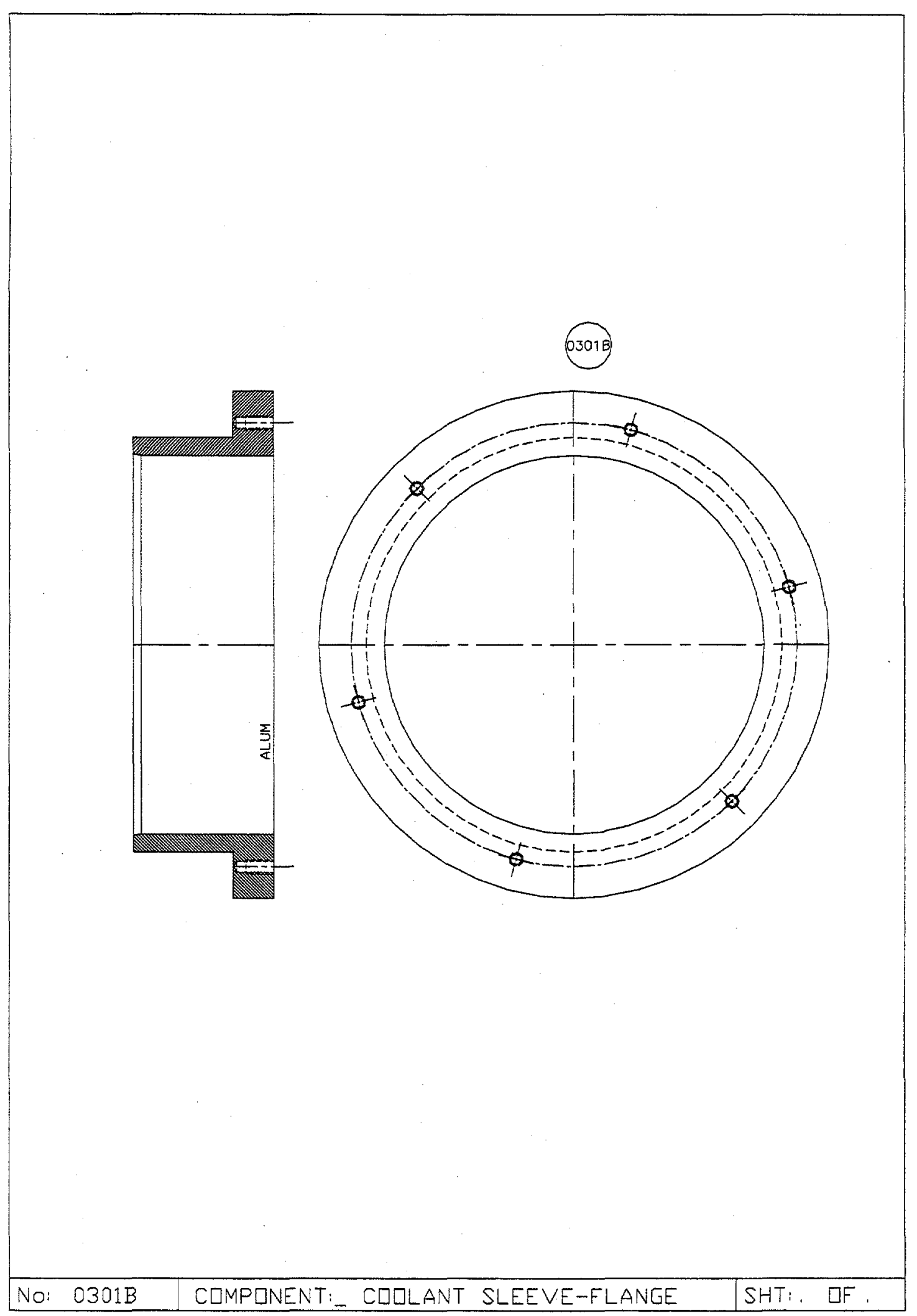

Drawing 14. Motor cooling sleeve - clamping flange. 
67

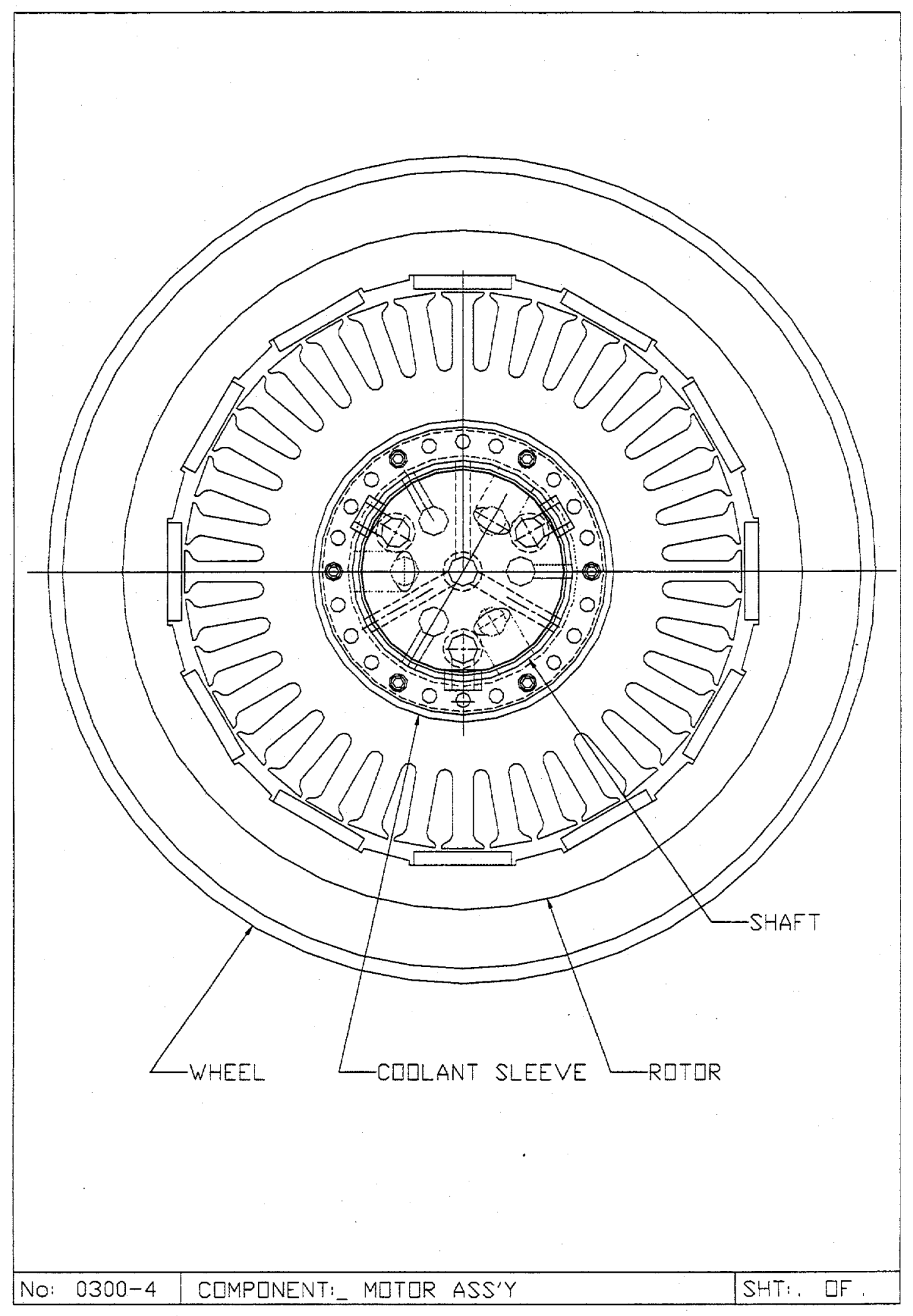

\section{Drawing 15. Spindle assembly end view (motor, shaft, cooling sleeve, wheel).}




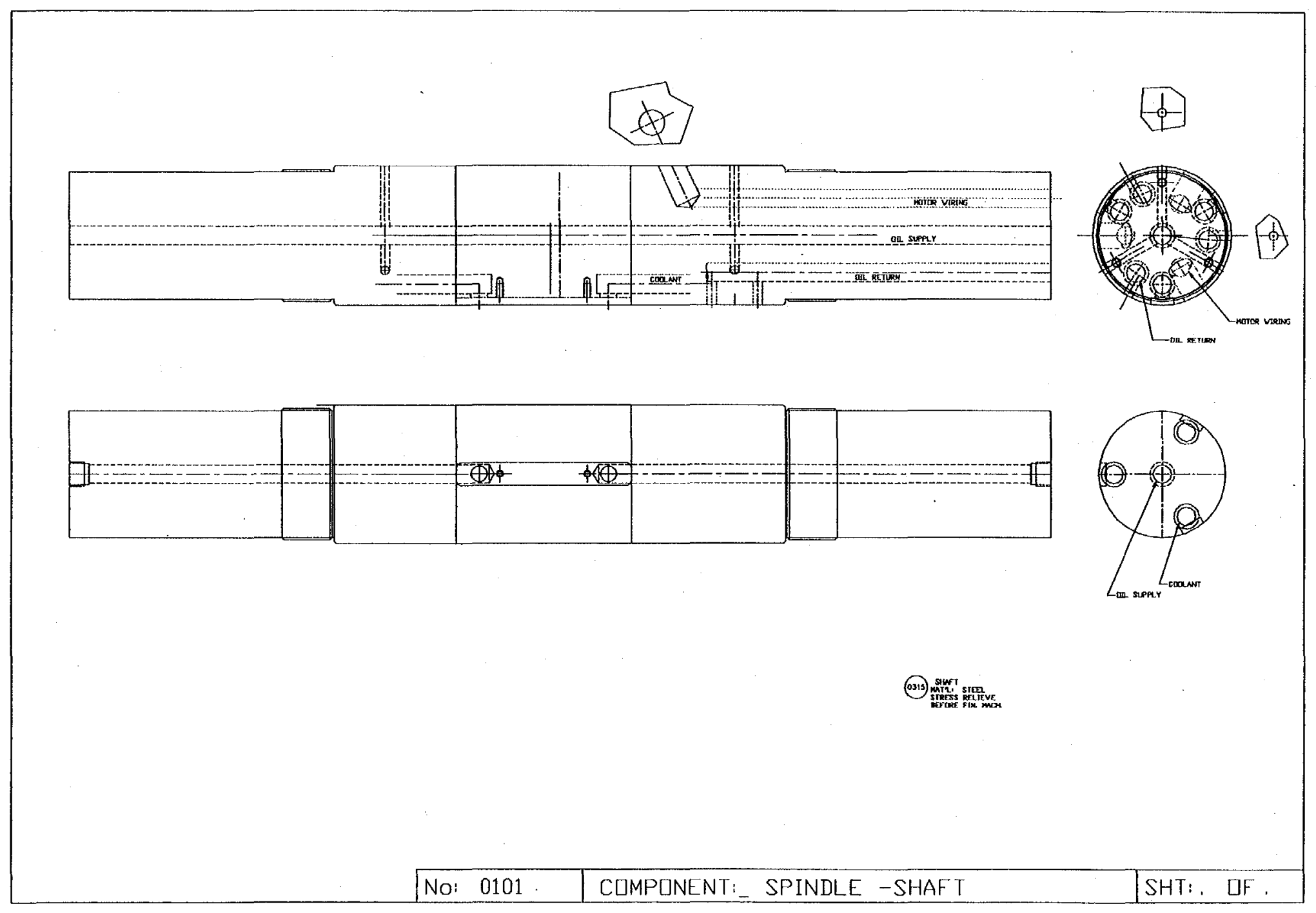

$\infty$

Drawing 16. Shaft detail illustrating conduits for bearing oil, cooling water, and motor leads. 
69

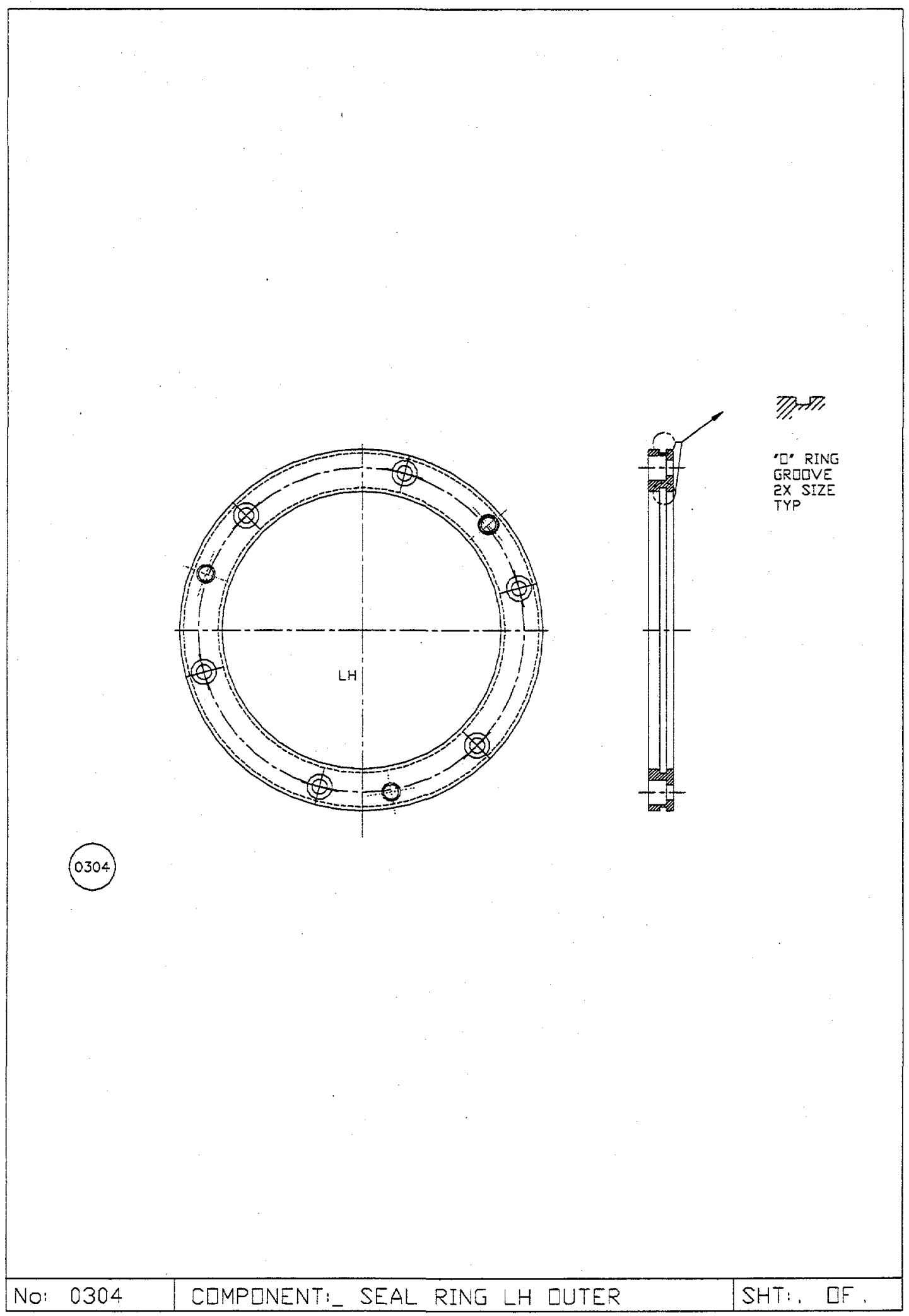

Drawing 17. Cooling sleeve sealing ring. 

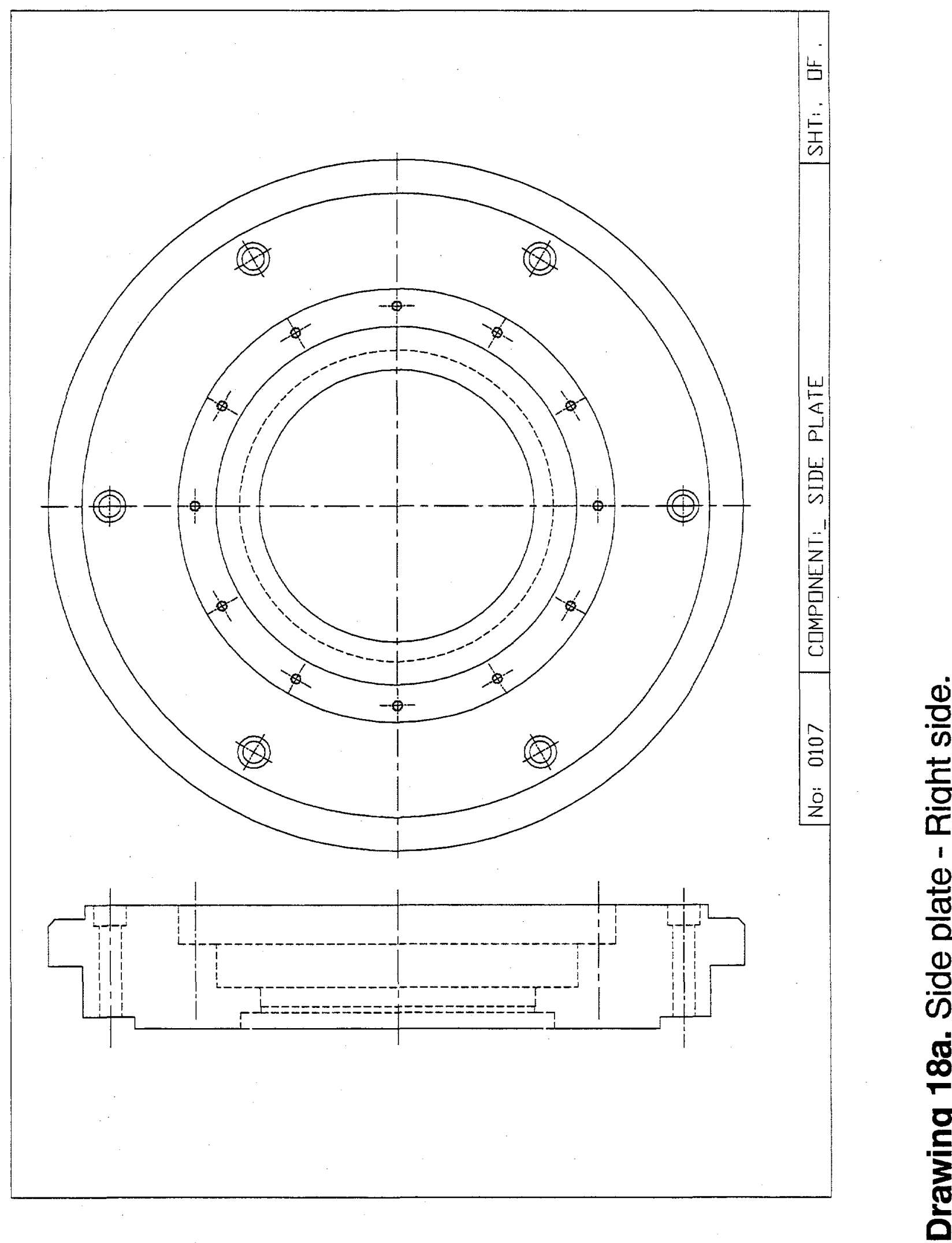


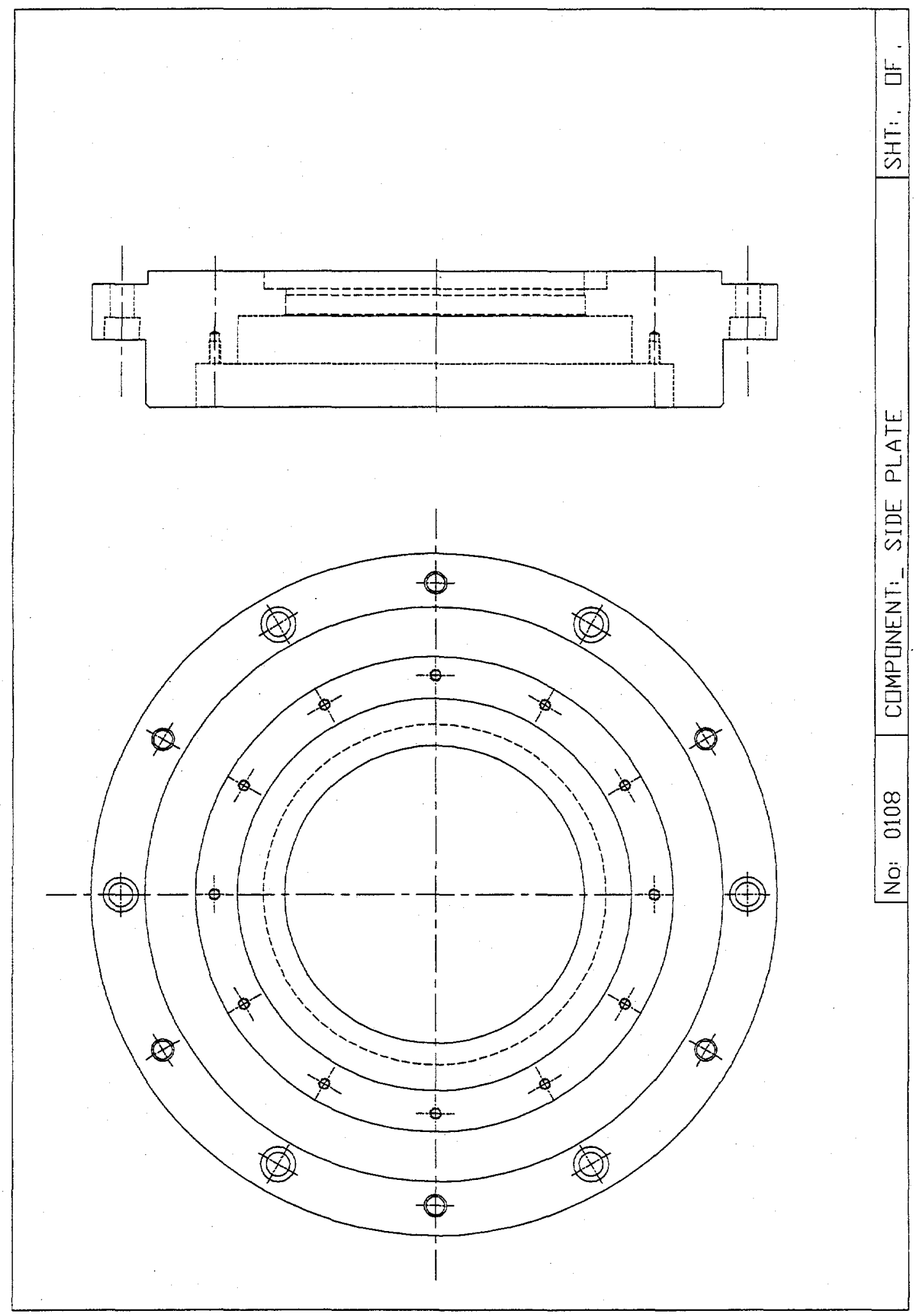

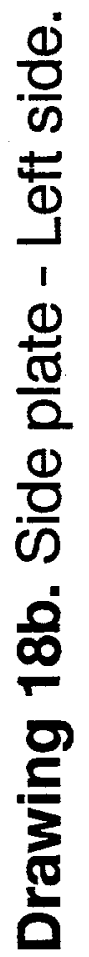



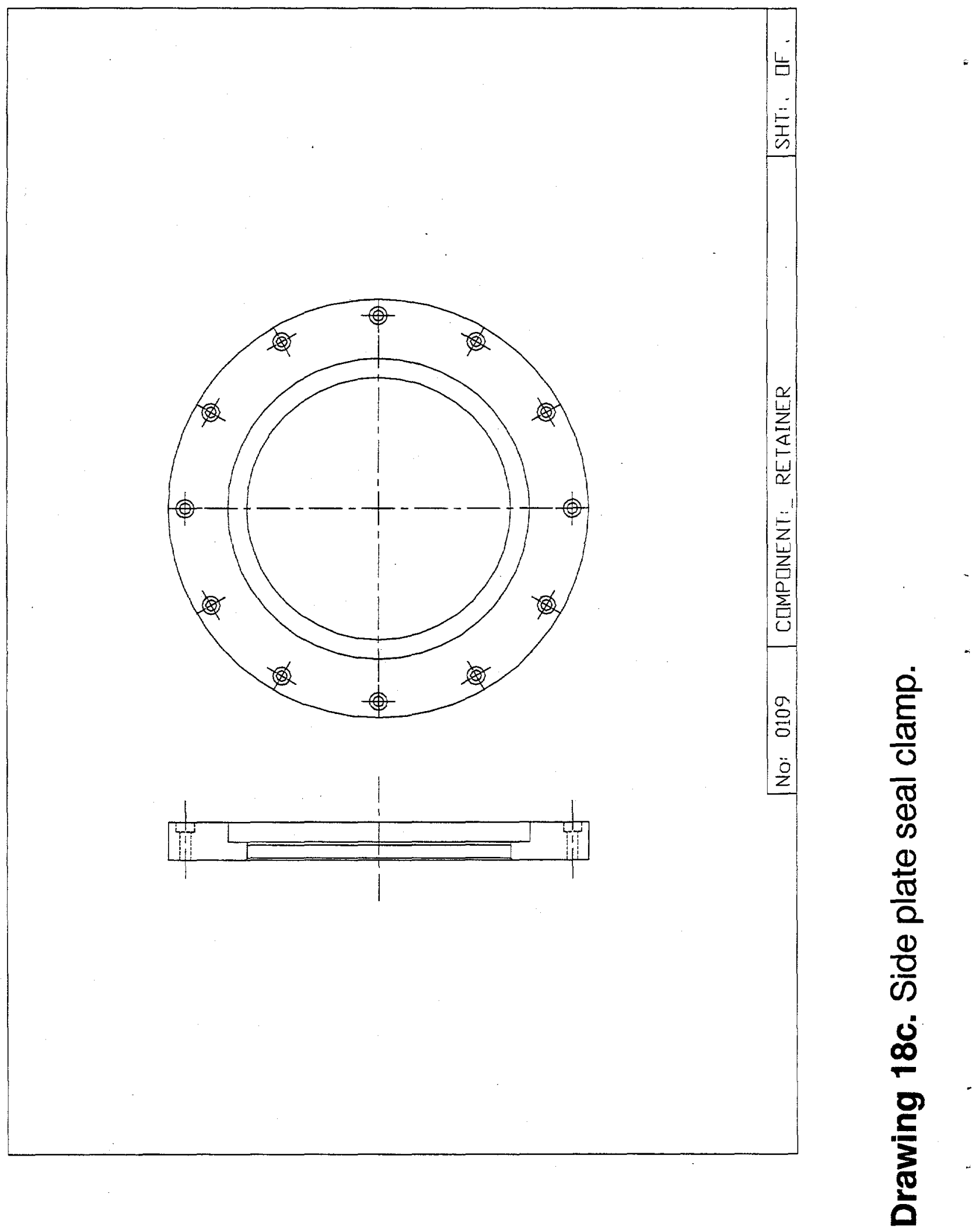


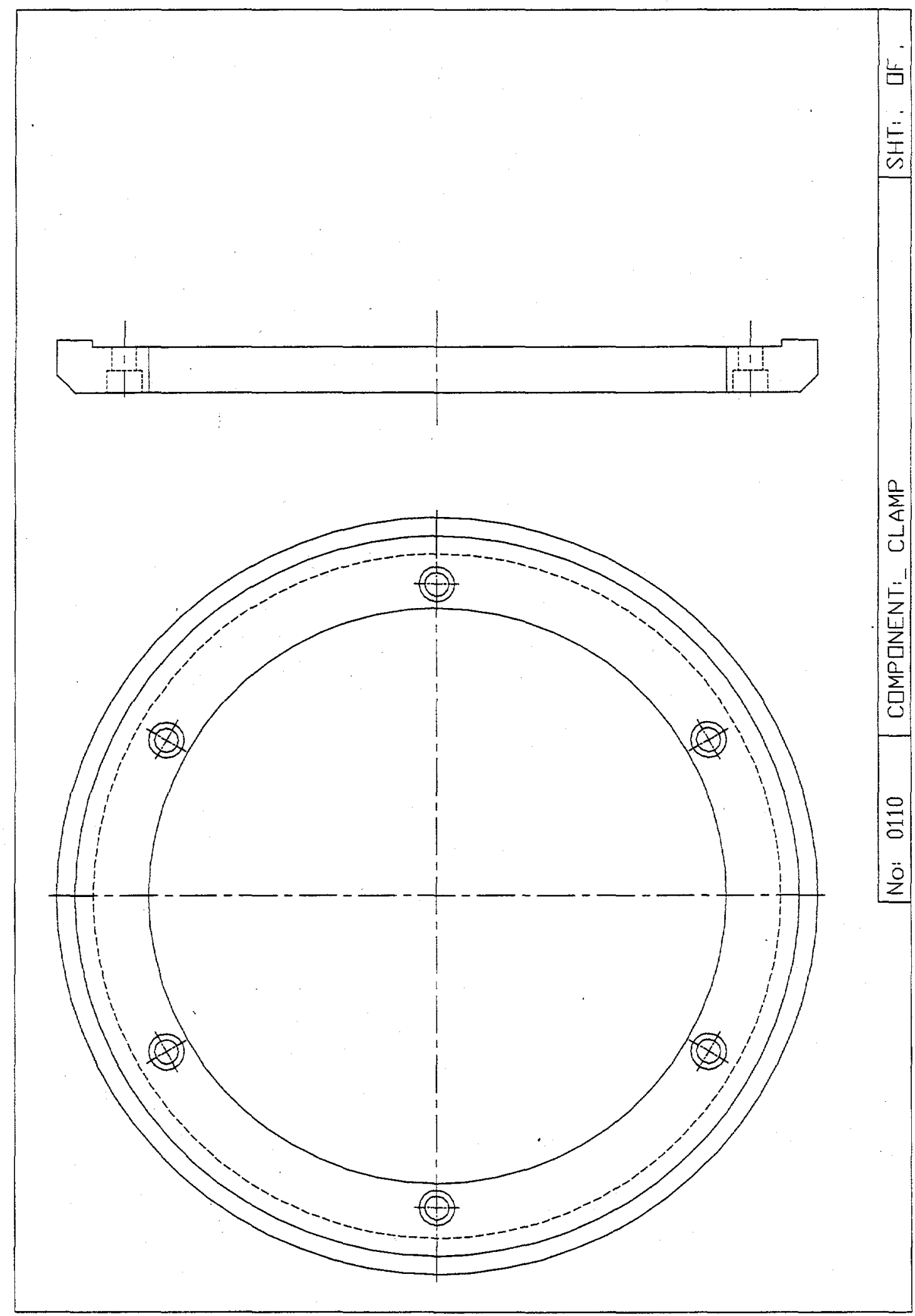




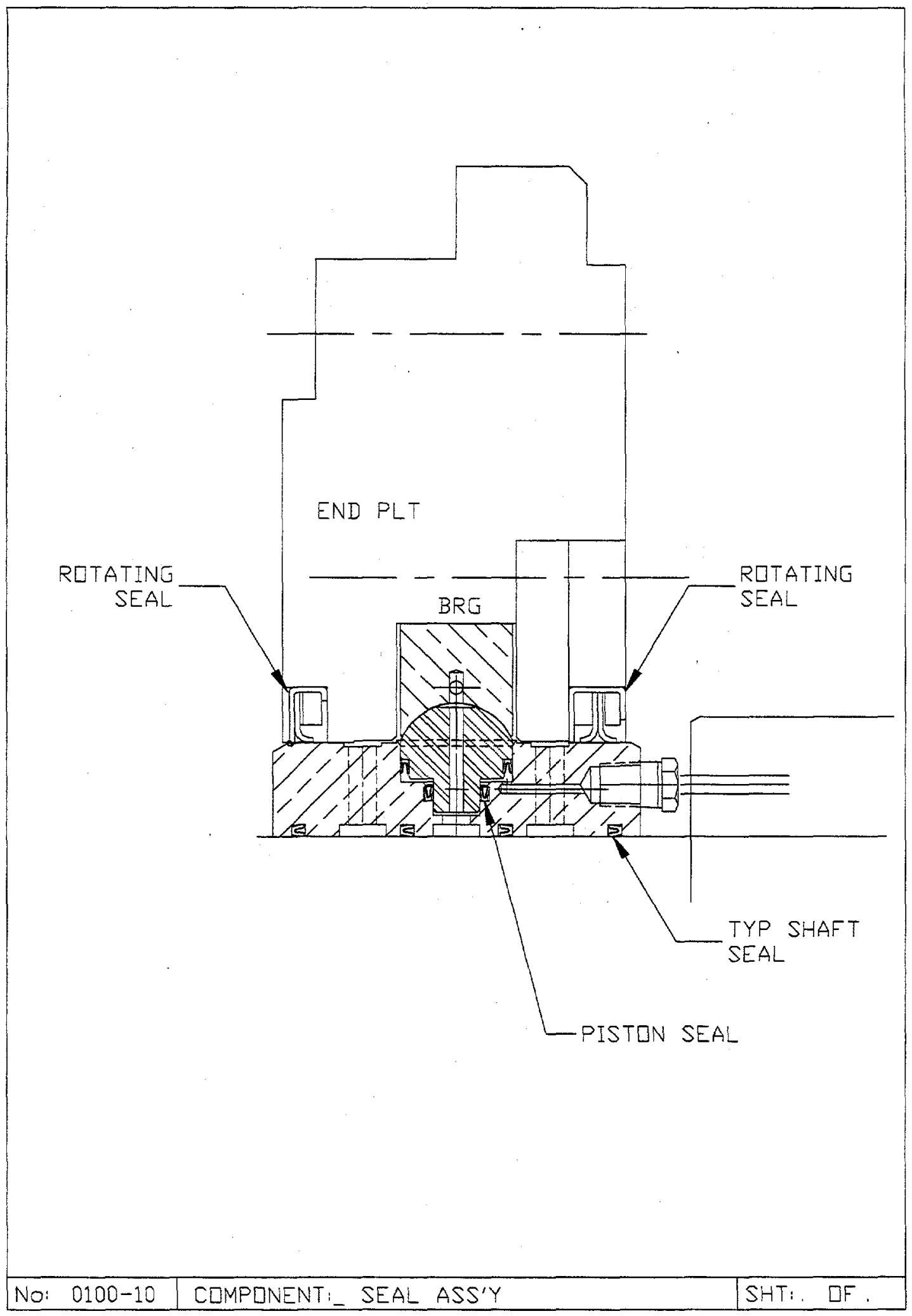

Drawing 19. Assembly view of end plate, bearing, actuator, and seals. 
INTERNAL DISTRIBUTION

L. F. Allard, Jr.

P. F. Becher

T. M. Besmann

P. J. Blau

R. A. Bradley

K. Breder

C. R. Brinkman

T. D. Burchell

A. Choudhury

D. D. Conger

S. A. David

M. K. Ferber

R. L. Graves

C. R. Hubbard

M. A. Janney

D. R. Johnson (5)
R. R. Judkins

M. A. Karnitz

R. J. Lauf

K. C. Liu

W. D. Manly

S. B. McSpadden

T. A. Nolan

A. E. Pasto

M. H. Rawlins

A. C. Schaffhauser

D. P. Stinton

T. N. Tiegs

S. G. Winslow

R. E. Ziegler

Laboratory Records - RC 
Jeffrey Abboud

U.S. Advanced Ceramics Assoc. 1600 Wilson Blvd., Suite 1008

Arlington VA 22209

B. P. Bandyopadhyay University of North Dakota Box 8359 University Station

Grand Forks ND 58202-8359

Donald F. Baxter, Jr.

Advanced Materials \& Processes

ASM International

9639 Kinsman Road

Materials Park OH 44073-0002

M. Brad Beardsley

Caterpillar Inc.

Technical Center Bldg. E

P.O. Box 1875

Peoria IL 61656-1875

Ramakrishna T. Bhatt

NASA Lewis Research Center

MS-106-1

21000 Brookpark Road

Cleveland, OH 44135

Bruce Boardman

Deere \& Company, Technical Ctr.

3300 River Drive

Moline II 61265-1792

Michael C. Brands

Cummins Engine Company, Inc.

P.O. Box 3005, Mail Code 50179

Columbus IN 47201

Donald J. Bray

Advanced Refractory Technologies

699 Hertel Avenue

Buffalo NY 14207
Jeff Bougher

Caterpillar Inc.

Technical Center, Bldg. E

P.O. Box 1875

Peoria IL 61656-1875

Mike Bowling

Cummins Engine Company, Inc.

1900 McKinley Avenue

P.O. Box 3005

Columbus IN 47202-3005

Walter Bryzik

U.S. Army Tank Automotive

Command

R\&D Center, Propulsion Systems

Warren MI 48397-5000

David Carruthers

Kyocera Industrial Ceramics

5713 East Fourth Plain

Vancouver WA 98661

Ronald H. Chand

Morton Advanced Materials

185 New Boston Street

Woburn MA 01801

William J. Chmura

Torrington Company

59 Field Street, P.O. Box 1008

Torrington CT 06790-1008

William S. Coblenz

Defense Adv. Research Projects Agency

3701 N. Fairfax Drive

Arlington VA 22203-1714

Gloria M. Collins

ASTM

100 Barr Harbor Drive

West Conshohocken PA 19428-2959

Shawn Cooper

FEV Engine Technology

4554 Glenmeade Lane

Auburn Hills MI 48326-1766 
Douglas Corey

AlliedSignal, Inc.

2525 West 190th Street, MS:T52

Torrance CA 90504-6099

Keith P. Costello

Chand/Kare Technical Ceramics

2 Coppage Drive

Worcester MA 01603-1252

Gary M. Crosbie

Ford Motor Company

P.O. Box 2053, 20000 Rotunda Drive

MD-3182, SRL Building

Dearborn MI 48121-2053

Pamela Cunningham

WETO Technical Library

MSE, Inc.

Industrial Park, P. O. Box 4078

Butte MT 59702

S. Keoni Denison

Norton Company

1 New Bond Street

Worcester MA 01615-0008

Sidney Diamond

U.S. Department of Energy

Office of Transportation Technologies

EE-33, Forrestal Building

Washington DC 28505

Ernest J. Duwell

3M Abrasive Systems Division

3M Center, Bldg. 251-01-34

St. Paul MN 55144

Michael Easley

AlliedSignal Engines

P. O. Box 52181

MS 551-11

Phoenix AZ 85072-2181
James J. Eberhardt

U.S. Department of Energy

Office of Transportation Technologies

EE-33, Forrestal Building

Washington DC 20585

Jim Edler

Eaton Corporation

26201 Northwestern Highway

P.O. Box 766

Southfield MI 48037

William A. Ellingson

Argonne National Laboratory

Energy Technology Division, Bldg. 212

9700 S. Cass Avenue

Argonne Il 60439-3848

John W. Fairbanks

U.S. Department of Energy

Office of Transportation Technologies

EE-33, Forrestal Building

Washington DC 20585

Ho Fang

Applied Materials

2695 Augustine Drive, MS-0962

Santa Clara CA 95054

Dan Foley

AlliedSignal Ceramic Components

MS: $1 / 5-1,26000$

2525 West 190th Street

Torrance CA 90504

Douglas Freitag

DuPont Lanxide Composites

21150 New Hampshire Avenue

Brookeville MD 20833

Richard Gates

NIST

Bldg. 223, Rm. A-256

Rt. 270 \& Quince Orchard Road

Gaithersburg MD 20899 
Ludwig J. Gauckler

ETH Zurich

Nonmetallic Materials

Sonneggstr. 5

CH-8092 Zurich, SWITZERLAND

Allan E. Goldman

U.S. Graphite, Inc.

907 W. Outer Drive

Oak Ridge TN 37830

Robert J. Gottschall

U.S. Department of Energy

Metal \& Ceramic Sciences, ER-131

19901 Germantown Road

Germantown MD 20874-1290

Thomas J. Gross

U.S. Department of Energy

Office of Transportation Technologies

EE-30, Forrestal Building

Washington DC 20585

Changsheng Guo

United Technologies Research Center

Machining Systems, MS 129-46

East Hartford CT 06108

Darryl Gust

Cummins Engine Company, Inc.

$1900 \mathrm{McKinley}$ Avenue

P.O. Box 3005

Columbus IN 47202-3005

Nabil S. Hakim

Detroit Diesel Corporation

13400 Outer Drive West, A08

Detroit MI 48239-4001

Alan M. Hart

Dow Chemical Company

1776 Building

Midland MI 48674
Michael H. Haselkorn

Caterpillar Inc.

Technical Center, Building E

P.O. Box 1875

Peoria IL 61656-1875

Deborah A. Haught

U.S. Department of Energy

Ofc. of Industrial Crosscut Technologies

EE-23, Forrestal Bldg.

Washington DC 20585

Daniel Hauser

Edison Welding Institute

Microjoinint \& Plastics Tech. Team

1250 Arthur E. Adams Drive

Columbus OH 43221-3585

John Haygarth

Wah Chang

P.O. Box 460

Albany OR 97321-0460

Gene Huber

Precision Ferrites \& Ceramics

5432 Production Drive

Huntington Beach CA 92649-1525

Thomas A. Johnson

Lanxide Corporation

1300 Marrows Road

P.O. Box 6077

Newark DE 19714-6077

Adam Jostsons

ANSTO

PMB1

Menai, NSW, Australia 2234

Yury Kalish

Detroit Diesel Corporation

Mechanical Systems

13400 Outer Drive West

Detroit MI 48239-4001 
Roy Kamo

Adiabatics, Inc.

3385 Commerce Park Drive

Columbus IN 47201

Ralph Kelly

Cincinnati Milacron

P.O. Box 9013

Cincinnati OH 45209

W. C. King

Mack Truck, Z-41

1999 Pennsylvania Avenue

Hagerstown MD 21740

Tony Kirn

Caterpillar Inc.

Defense Products Dept., JB7

Peoria II 61629

Joseph A. Kovach

Parker Hannifin Corporation

6035 Parkland Boulevard

Cleveland OH 44124-4141

Edwin H. Kraft

Kyocera Industrial Ceramics

5713 E. Fourth Plain Boulevard

Vancouver WA 98661

Oh-Hun Kwon

Norton Company

Saint Gobain Industrial Ceramics

1 Goddard Road

Northboro MA 01532-1545

S. K. Lau

B. F. Goodrich Aerospace R\&D

9921 Brecksville Road

Brecksville OH 44141

Elaine Lentini

Saint-Gobain Industrial Ceramics

Goddard Road

Northboro MA 01532
Stan Levine

NASA Lewis Research Center

21000 Brookpark Road, MS:106/5

Cleveland $\mathrm{OH} 44135$

Robert H. Licht

Norton Company

Saint Gobain Industrial Ceramics

1 Goddard Road

Northboro MA 01532-1545

E. Lilley

Norton Company

Saint Gobain Industrial Ceramics

1 Goddard Road

Northboro MA 01532-1545

B. J. McEntire

Applied Materials Corporation

3050 Bowers Avenue

Santa Clara, CA 95054

James McLaughlin

Sundstrand Power Systems

4400 Ruffin Road

P.O. Box 85757

San Diego CA 92186-5757

Biljana Mikijelj

Ceradyne, Inc.

3169 Red Hill Avenue

Costa Mesa CA 92626

Carl E. Miller

Delphi Energy \& Engine Mgmt. Systems

4800 S. Saginaw Street, MC 485-301-150

P. O. Box 1360

Flint MI 48501-1360

Curtis V. Nakaishi

U.S. Department of Energy

Federal Energy Tech. Center

3610 Collins Ferry Rd.

P.O. Box 880

Morgantown WV 26507-0880 
Malcolm Naylor

Cummins Engine Company, Inc.

P.O. Box 3005, Mail Code 50183

Columbus IN 47202-3005

Dale E. Niesz

Ceramic \& Materials Engineering

607 Taylor Road, Rm. 204

Piscataway, NJ 08854-8065

Thomas J. Paglia

Coors/ACI

3315 Boone Road

Benton AR 72015

Richard Palicka

CERCOM, Inc.

1960 Watson Way

Vista CA 92083

Vijay M. Parthasarathy

Solar Turbines

2200 Pacific Highway, M.Z. R-1

San Diego CA 92186

Magan Patel

Cummins Engine Company, Inc.

Mail Code 50183

Box 3005

Columbus IN 47202-3005

James W. Patten

Cummins Engine Company, Inc.

P.O. Box 3005, Mail Code 50183

Columbus IN 47202-3005

Joe Picone

Norton Company

1 New Bond Street

Box 15008

Worcester MA 01615-0008

Stephen C. Pred

Pred Materials International, Inc. 60 East 42nd Street, Suite 1456

New York NY 10165
Vimal K. Pujari

Norton Company

Saint Gobain Industrial Ceramics

1 Goddard Road

Northboro MA 01532-1545

Fred Quan

Corning Inc.

Sullivan Park, FR-2-8

Corning NY 14831

George Quinn

NIST

I-270 \& Clopper Road

Ceramics Division, Bldg. 223

Gaithersburg MD 20899

Mike Readey

Caterpillar, Inc.

Technical Center, Bldg. E

P.O. Box 1875

Peoria IL 61656-1875

Harold Rechter

Chicago Fire Brick Company

7531 S. Ashland Avenue

Chicago II 60620-4246

Jack A. Rubin

CERCOM, Inc.

1960 Watson Way

Vista CA 92083

Robert J. Russell

Riverdale Consulting, Inc.

24 Micah Hamlin Road

Centerville MA 02632-2107

J. Sankar

North Carolina A\&T State Univ.

Dept. of Mechanical Engineering

Greensboro NC 27406 
Maxine L. Savitz

AlliedSignal, Inc.

Ceramic Components

2525 West 190th Street

P.O. Box 2960, MS:1/5-1, 26000

Torrance CA 90509-2960

Jim Schienle

AlliedSignal Aerospace

1130 West Warner Road

M/S 1231-K

Tempe AZ 85284

Gary Schnittgrund

Transfer Technology

16401 Knollwood Drive

Granada Hills CA 91344

Robert S. Shane

Shane Associates

1904 NW 22nd Street

Stuart FL 34994-9270

Subu Shanmugham

MicroCoating Technologies

3901 Green Industrial Way

Chamblee GA 30341-1913

Albert J. Shih

North Carolina State University

Mechanical \& Aerospace Engineering

2217 Broughton Hall, Box 7910

Raleigh NC 27695

Charles Spuckler

NASA Lewis Research Center

21000 Brookpark Road, MS: 5-11

Cleveland $\mathrm{OH} 44135-3127$

Gordon L. Starr

Cummins Engine Company, Inc.

P.O. Box 3005, Mail Code:50182

Columbus IN 47202-3005
Marian Swirsky

Cambridge Scientific Abstract

Commerce Park, Bldg. 4, Suite 804

23200 Chagrin Blvd.

Beachwood OH 44122

Victor J. Tennery

113 Newell Lane

Oak Ridge TN 37830

Malcolm Thomas

Allison Engine Company

P. O. Box 420 (W06)

Indianapolis IN 46206

Marc Tricard

Norton Company

Superabrasives Division

1 New Bond Street, MS-412-301

P. O. Box 15008

Worcester MA 01615-0008

Marcel H. Van De Voorde

Commission of the European Union

Eeuwigelaan 33

1861 CL Bergen

THE NETHERLANDS

V. Venkateswaran

Materials Solutions International, Inc.

P.O. Box 663

Grand Island, NY 14072-0663

Robert M. Washburn

ASMT

11203 Colima Road

Whittier CA 90604

R. W. Weeks

Argonne National Laboratory

Bldg. 362, E313

9700 S. Cass Avenue

Argonne IL 60439 
Sheldon M. Wiederhorn

NIST

Building 223, Room B309

Gaithersburg MD 20899

Matthew F. Winkler

Seaworthy Systems, Inc.

P.O. Box 965

Essex CT 06426

Thomas J. Wissing

Eaton Corporation

26201 Northwestern Highway

P.O. Box 766

Southfield MI 48037

James C. Withers

MER Corporation

7960 S. Kolb Road

Tucson AZ 85706

Dale E. Wittmer

Southern Illinois University

Mechanical Engineering Dept.

Carbondale II 62901

Egon E. Wolff

Caterpillar Inc.

Technical Center

P.O. Box 1875

Peoria IIL 61656-1875

Roy Yamamoto

Ethyl Petroleum Additives, Inc.

500 Spring Street

P. O. Box 2158

Richmond VA 23218-2158

R. L. Yeckley

Kennametal, Inc.

P.O. Box 231

Latrode, PA 15650
Thomas M. Yonushonis

Cummins Engine Company, Inc.

1900 McKinley Avenue

P.O. Box 3005, Mail Code 50183

Columbus IN 47202-3005

S. Charles Yoon

Cincinnati Milacron, Inc.

P.O. Box 9013

3000 Disney Street

Cincinnati OH 45209-9013

Jong Yung

Sundstrand Aerospace

Dept. 789-6

4747 Harrison Avenue

Rockford IL 61125

A. L. Zadoks

Caterpillar Inc.

Technical Center, Building L

P.O. Box 1875

Peoria IL 61656-1875

Zhenqi Zhu

Stevens Institute of Technology

Department of Mechanical Engineering

Castle Point on Hudson

Hoboken NJ 07030

Department of Energy

Oak Ridge Operations Office

Assistant Manager for Energy

Research and Development

P. O. Box 2001

Oak Ridge TN $37831-8600$

For distribution by microfiche as shown in DOE/OSTI-4500,

Distribution Category UC-332

(Ceramics/Advanced Materials). 